\title{
UV-TO-FIR ANALYSIS OF SPITZER/IRAC SOURCES IN THE EXTENDED GROTH STRIP. II. PHOTOMETRIC REDSHIFTS, STELLAR MASSES, AND STAR FORMATION RATES
}

\author{
G. Barro ${ }^{1}$, P. G. Pérez-González ${ }^{1,5}$, J. Gallego ${ }^{1}$, M. L. N. Ashby ${ }^{2}$, M. Kajisawa ${ }^{3}$, S. Miyazaki ${ }^{4}$, V. Villar ${ }^{1}$, \\ T. YAMADA ${ }^{3}$, AND J. ZAMORANO ${ }^{1}$ \\ ${ }^{1}$ Departamento de Astrofísica, Facultad de CC. Físicas, Universidad Complutense de Madrid, E-28040 Madrid, Spain \\ ${ }^{2}$ Harvard-Smithsonian Center for Astrophysics, 60 Garden St., Cambridge, MA 02138, USA \\ ${ }^{3}$ Astronomical Institute, Tohoku University, Aramaki, Aoba, Sendai 9808578, Japan \\ ${ }^{4}$ National Astronomical Observatory of Japan, Mitaka, Tokyo 181-8588, Japan \\ Received 2010 May 23; accepted 2011 February 19; published 2011 March 31
}

\begin{abstract}
Based on the ultraviolet to far-infrared photometry already compiled and presented in a companion paper (Paper I), we present a detailed spectral energy distribution (SED) analysis of nearly 80,000 IRAC 3.6 +4.5 $\mu \mathrm{m}$ selected galaxies in the Extended Groth Strip. We estimate photometric redshifts, stellar masses, and star formation rates (SFRs) separately for each galaxy in this large sample. The catalog includes 76,936 sources with [3.6] $\leqslant 23.75$ (85\% completeness level of the IRAC survey) over $0.48 \mathrm{deg}^{2}$. The typical photometric redshift accuracy is $\Delta z /(1+z)=$ 0.034 , with a catastrophic outlier fraction of just $2 \%$. We quantify the systematics introduced by the use of different stellar population synthesis libraries and initial mass functions in the calculation of stellar masses. We find systematic offsets ranging from 0.1 to $0.4 \mathrm{dex}$, with a typical scatter of $0.3 \mathrm{dex}$. We also provide UV- and IR-based SFRs for all sample galaxies, based on several sets of dust emission templates and SFR indicators. We evaluate the systematic differences and goodness of the different SFR estimations using the deep FIDEL $70 \mu \mathrm{m}$ data available in the Extended Groth Strip. Typical random uncertainties of the IR-bases SFRs are a factor of two, with non-negligible systematic effects at $z \gtrsim 1.5$ observed when only MIPS $24 \mu \mathrm{m}$ data are available. All data products (SEDs, postage stamps from imaging data, and different estimations of the photometric redshifts, stellar masses, and SFRs of each galaxy) described in this and the companion paper are publicly available, and they can be accessed through our the Web interface utility Rainbow-navigator.
\end{abstract}

Key words: galaxies: high-redshift - galaxies: photometry - galaxies: starburst - infrared: galaxies

Online-only material: color figures, machine-readable tables

\section{INTRODUCTION}

Multi-band catalogs are the fuel for studies aimed at exploring the global evolution of galaxies over cosmic history. They have been used to study the redshift evolution of the star formation rate (SFR) density (e.g., Hopkins \& Beacom 2006; Reddy et al. 2008; Bouwens et al. 2009), and the stellar mass assembly process (e.g., Bundy et al. 2006; Fontana et al. 2006; PérezGonzález et al. 2008; Marchesini et al. 2009).

The unprecedented sensitivity of modern surveys detect millions of distant galaxies to faint flux levels that for all practical purposes lie well beyond the capabilities of even the most recent multi-object spectrographs at the largest telescopes. As a consequence, their intrinsic properties must be estimated through multi-band photometric data using fitting techniques to stellar population templates, and/or empirical relations. Among the basic parameters needed to characterize a galaxy, arguably the most important is the redshift, which must be inferred from an analysis of its spectral energy distribution (SED). Photometric redshift techniques are now sufficiently accurate to derive statistically reliable conclusions for high-redshift galaxy populations (e.g., Silva et al. 1998; Wolf et al. 2003; Ilbert et al. 2009).

Many different codes have been developed to calculate photometric redshifts based in the same principle: finding the galaxy spectral template best fitting the observed photometry in several band-passes. Some examples include HYPERZ (Bolzonella

\footnotetext{
5 Associate Astronomer at Steward Observatory, The University of Arizona, USA.
}

et al. 2000), BPZ (Benítez 2000), or LePHARE (e.g., Arnouts \& Ilbert; Ilbert et al. 2009). The implementation is very sensitive to the quality of the photometry and the capability of the observed bands to probe key continuum features of the spectra (e.g., the Lyman and Balmer breaks). It also depends strongly on the availability of templates that are statistically representative and successful in characterizing the emission of galaxies. The impact of these factors in the uncertainty of the estimations is not straightforward, and it can lead to catastrophic errors beyond the simple propagation of the statistical errors (Oyaizu et al. 2008; Hildebrandt et al. 2008). In recent years, several techniques have been developed to improve the reliability of the photometric redshifts (e.g., Bayesian priors (Benítez 2000), template-optimization procedures (Ilbert et al. 2006a), and machine-learning neural networks (Collister \& Lahav 2004)). Recent work including some of these advances have achieved remarkable precision [e.g., $\Delta z /(1+z)<0.012$ in Ilbert et al. 2009, and $\Delta z /(1+z)=0.06$ at $z>1.5$ in van Dokkum et al. 2009].

Once a galaxy's redshift has been estimated, the most significant physical properties that can then be derived from multiwavelength photometry are the stellar mass and the SFR. However, estimates derived from modeling of the observed SEDs involve significant random and systematic uncertainties. The estimate of the stellar mass by fitting stellar population synthesis (SPS) models is a widespread technique (e.g., Bell et al. 2003; Panter et al. 2007; Walcher et al. 2008) that requires making some assumptions regarding the initial stellar mass function, the star formation history, or the extinction law. Moreover, there 
exist significant differences among stellar population libraries. These differences can lead to discrepancies in the stellar mass estimation of a factor of a few (Maraston et al. 2006; Bruzual 2007).

SFR estimates based on UV and/or IR luminosities are considered reasonably robust for large galaxy samples with multi-wavelength photometry, where other tracers, such as spectroscopy, are unavailable (Reddy et al. 2006; Salim et al. 2007; Daddi et al. 2007). A major problem with SFRs estimated from UV data is the need for a extinction correction, which can be highly uncertain and redshift dependent (Iglesias-Páramo et al. 2007; Burgarella et al. 2007; Salim et al. 2009). On the other hand, IR-based SFRs estimated by fitting the MIR-tomm fluxes with dust emission templates are model dependent (Papovich \& Bell 2002; Dale et al. 2005; Caputi et al. 2006). Furthermore, these tracers are based on the assumption that the bulk of the IR emission traces warm dust heated by young starforming regions. Thus, if a fraction of the energy heating the dust originates from an alternative source, such as deeply dust enshrouded active galactic nuclei (AGNs) or diffuse radiation fields (Daddi et al. 2007; Salim et al. 2009) the SFR will be overestimated. Nevertheless, despite these second-order effects, the uncertainties in the SFR are frequently driven by the absence of sufficient IR photometry to constrain the models robustly. In the last few years, the studies of SFRs at high redshift have often been based on the observed flux at MIPS $24 \mu \mathrm{m}$ only and, although IR monochromatic luminosities are known to correlate well to total IR luminosity, recent works based on more detailed IR coverage have demonstrated that SFRs from MIPS $24 \mu \mathrm{m}$ data may present significant systematics (Papovich et al. 2007; Daddi et al. 2007; Rigby et al. 2008).

In this context, Barro et al. (2011, hereafter Paper I) presented a multi-band photometric and spectroscopic catalog (including data from X-ray to radio wavelengths) in the Extended Groth Strip (EGS) that can be used as a starting point for detailed analysis of the galaxy population. That paper describes the method used to measure coherent multi-band photometry and presents the general properties of the merged catalog, including an analysis of the quality and reliability of the photometry. Paper I also presents Rainbow Navigator, a publicly available Web interface that provides access to all the multi-band data products.

In this paper, we focus on fitting the optical-to-NIR SEDs and IR emission of all the sources presented in Paper I using SPS models and dust emission templates. We then use the SEDs and fits to estimated photometric redshifts, stellar masses, and SFRs. We also quantify the uncertainties attending these estimations. In particular, we assess the quality of the photometric redshifts by comparing our results with spectroscopic redshifts and with other photometric redshift compilations found in the literature. We explore the systematic uncertainties in the stellar masses associated with the modeling assumptions, such as the choice of SPS models or the initial mass function (IMF). Finally, we study the systematic uncertainties in the IR-based SFRs estimated with different IR templates and indicators (e.g., different total IR luminosity-to-SFR calibrations).

The outline of this paper is as follows. Section 2 briefly reviews the available data and then summarizes the most relevant steps of the photometric measurement and bandmerging procedure (presented in Paper I), as well as the overall photometric properties of the IRAC $3.6+4.5 \mu \mathrm{m}$ selected catalog. Section 3 describes the techniques developed to perform the UV-to-IR SED fitting, and the methods used to estimate

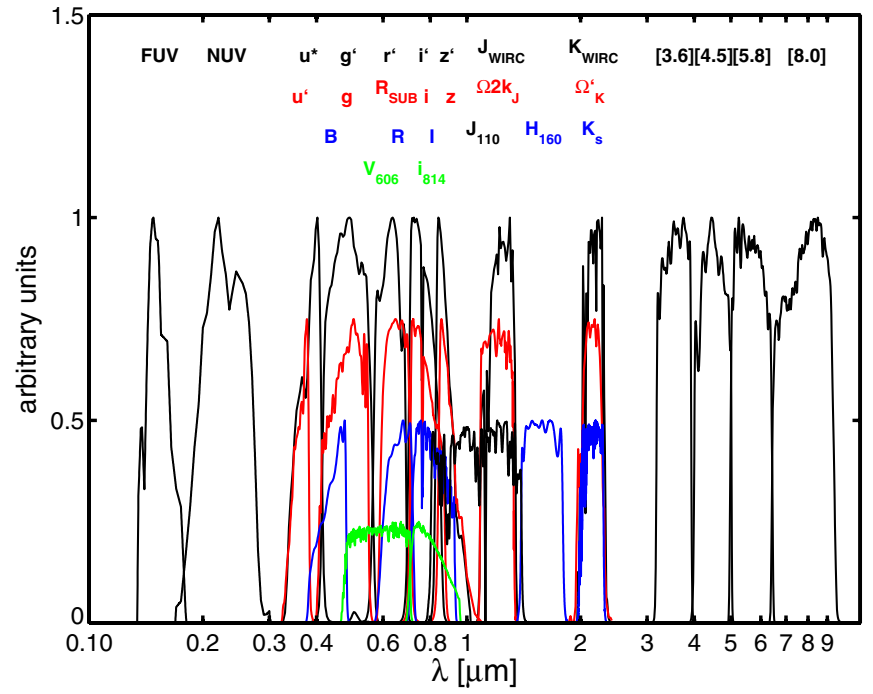

Figure 1. Filter transmission for the photometric bands included in the data set. The curves include the atmospheric transmission (for ground-based observations), quantum efficiency, and the transmission of the optical elements. The curves are normalized at the maximum value of the transmission and scaled arbitrarily for visualization. The color code for each filter corresponds to the labels shown above. The optical bands depicted from top to bottom are those of CFHTLS, MMT, and CFHT12k, respectively. The NIR bands, also shown from top-to-bottom are those of WIRC, CAHA, and HST/NICMOS. The GALEX (FUV, NUV) and IRAC ([3.6], [4.5], [5.8], [8.0]) filters are also listed in the top row.

(A color version of this figure is available in the online journal.)

redshifts. Section 4 describes the stellar masses estimation technique, and quantifies the uncertainties introduced by the modeling assumptions. Section 5 describes the methods used to fit the FIR emission to dust emission templates and the estimation of IR luminosities and SFRs. Section 6 presents tables containing all the data products presented in this paper, as well as the public database created to facilitate the access to these resources.

Throughout this paper we use $\mathrm{AB}$ magnitudes. We adopt the cosmology $H_{0}=70 \mathrm{~km}^{-1} \mathrm{~s}^{-1} \mathrm{Mpc}^{-1}, \Omega_{m}=0.3$, and $\Omega_{\lambda}=0.7$. Our default choice of SED modeling parameters are the PEGASE (Fioc \& Rocca-Volmerange 1997) library, a Salpeter (1955) IMF $\left(M \in[0.1-100] M_{\odot}\right)$, and a Calzetti et al. (2000) extinction law.

\section{MULTI-WAVELENGTH CATALOG}

The present work is based on the multi-wavelength catalog of IRAC $3.6+4.5 \mu \mathrm{m}$ selected galaxies in the EGS $(\alpha=$ $\left.14^{\mathrm{h}} 14^{\mathrm{m}}, \delta=+53^{\circ} 30^{\prime}\right)$ presented in Paper I. The catalog contains all the publicly available data provided by the AllWavelength Extended Groth Strip International Survey (AEGIS) collaboration and some proprietary data including the following bands: GALEX, FUV, and NUV, CFHTLS $u^{*} g^{\prime} r^{\prime} i^{\prime} z^{\prime}$, MMT$u^{\prime}$ giz, CFHT12k BRI, ACS $V_{606} i_{814}$, Subaru R, NICMOS $J_{110} H_{160}$, MOIRCS $K_{s}$, CAHA- $J K_{s}$, WIRC $J K$, the four IRAC bands at 3.6, 4.5, 5.8, and $8.0 \mu \mathrm{m}$, and lastly MIPS 24 and $70 \mu \mathrm{m}$. We cross-correlated our IRAC-selected catalog with the X-ray (Chandra) and radio (VLA/20 cm) catalogs of Laird et al. (2009) and Ivison et al. (2007), and with all the spectroscopic redshifts from DEEP2 DR3 and a small sample of 238 spectroscopically confirmed Lyman break galaxies (LBGs) from Steidel et al. (2003). The reader is referred to Paper I and Davis et al. 2007, and references therein, for a detailed description of all these data sets. Figure 1 illustrates the different 
Table 1

Photometric Properties of the Data set

\begin{tabular}{|c|c|c|c|c|c|}
\hline $\begin{array}{l}\text { Filter } \\
(1)\end{array}$ & $\begin{array}{l}\lambda_{\text {eff }} \\
(2)\end{array}$ & $m_{\lim }[\mathrm{AB}]$ & $\begin{array}{c}\text { FWHM } \\
\text { (4) }\end{array}$ & $\begin{array}{l}\text { Gal. Ext } \\
\text { (5) }\end{array}$ & $\begin{array}{c}\text { Offset } \\
(6)\end{array}$ \\
\hline GALEX-FUV & $153.9 \mathrm{~nm}$ & 25.6 & $5 . .5$ & 0.195 & 0.04 \\
\hline GALEX-NUV & $231.6 \mathrm{~nm}$ & 25.6 & 5.5 & 0.101 & 0.08 \\
\hline ММТ- $u$ & $362.5 \mathrm{~nm}$ & 26.1 & $1^{\prime \prime} 0$ & 0.049 & -0.09 \\
\hline CFHTLS $-u^{*}$ & $381.1 \mathrm{~nm}$ & 25.7 & 0.9 & 0.045 & -0.04 \\
\hline CFHT-B & $439.0 \mathrm{~nm}$ & 25.7 & $1^{\prime \prime} .2$ & 0.036 & 0.04 \\
\hline MMT- $g$ & $481.4 \mathrm{~nm}$ & 26.7 & $1^{\prime \prime} 3$ & 0.031 & -0.09 \\
\hline CFHTLS- $g^{\prime}$ & $486.3 \mathrm{~nm}$ & 26.5 & 0.9 & 0.031 & 0.03 \\
\hline ACS- $V_{606}$ & $591.3 \mathrm{~nm}$ & 26.1 & $0 . .2$ & 0.022 & 0.02 \\
\hline CFHTLS- $r^{\prime}$ & $625.8 \mathrm{~nm}$ & 26.3 & $0^{\prime \prime} 8$ & 0.020 & 0.03 \\
\hline Subaru-R & $651.8 \mathrm{~nm}$ & 26.1 & 0.7 & 0.019 & 0.00 \\
\hline CFHT-R & $660.1 \mathrm{~nm}$ & 25.3 & $1^{\prime \prime} .0$ & 0.019 & -0.03 \\
\hline CFHTLS- $i^{\prime}$ & $769.0 \mathrm{~nm}$ & 25.9 & $0^{\prime \prime} 8$ & 0.015 & 0.03 \\
\hline MMT- $i$ & $781.5 \mathrm{~nm}$ & 25.3 & $1^{\prime \prime} 0$ & 0.015 & 0.00 \\
\hline ACS- $i_{814}$ & $813.2 \mathrm{~nm}$ & 26.1 & $0 . .2$ & 0.014 & 0.00 \\
\hline CFHT-I & $833.0 \mathrm{~nm}$ & 24.9 & $1^{\prime \prime} 1$ & 0.013 & 0.02 \\
\hline CFHTLS- $z^{\prime}$ & $887.1 \mathrm{~nm}$ & 24.7 & $0 . ! 8$ & 0.012 & -0.02 \\
\hline MMT- $z$ & $907.0 \mathrm{~nm}$ & 25.3 & $1^{\prime \prime} .2$ & 0.011 & -0.11 \\
\hline NICMOS- $J_{110}$ & $1.10 \mu \mathrm{m}$ & 23.5 & 0.7 & 0.008 & 0.00 \\
\hline$\Omega 2 k-J$ & $1.21 \mu \mathrm{m}$ & 22.9 & $1^{\prime \prime} .0$ & 0.007 & -0.16 \\
\hline WIRC- $J^{\mathrm{a}}$ & $1.24 \mu \mathrm{m}$ & 21.9 & $1^{\prime \prime} .0$ & 0.007 & 0.01 \\
\hline NICMOS- $H_{160}$ & $1.59 \mu \mathrm{m}$ & 24.2 & $0 . .8$ & 0.005 & 0.00 \\
\hline$\Omega^{\prime}-K$ & $2.11 \mu \mathrm{m}$ & 20.7 & $1^{\prime \prime} .5$ & 0.003 & -0.10 \\
\hline Subaru-MOIRCS- $K s$ & $2.15 \mu \mathrm{m}$ & 23.7 & 0.6 & 0.003 & -0.04 \\
\hline WIRC- $K^{\mathrm{a}}$ & $2.16 \mu \mathrm{m}$ & 22.9 & $1^{\prime \prime} 0$ & 0.003 & 0.00 \\
\hline IRAC-36 & $3.6 \mu \mathrm{m}$ & 23.7 & 2 .' 1 & 0.001 & 0.00 \\
\hline IRAC-45 & $4.5 \mu \mathrm{m}$ & 23.7 & $2^{\prime \prime} .1$ & 0.001 & 0.00 \\
\hline IRAC-58 & $5.8 \mu \mathrm{m}$ & 22.1 & 2.2 & 0.001 & 0.12 \\
\hline IRAC-80 & $8.0 \mu \mathrm{m}$ & 22.1 & $2^{\prime \prime} .2$ & 0.000 & 0.12 \\
\hline
\end{tabular}

Notes.

a The photometry was not measured, but taken from a published catalog. Column 1: name of the observing band and instrument.

Column 2: effective wavelength of the filter calculated by convolving the Vega spectrum (Colina \& Bohlin 1994) with the transmission curve of the filter+detector.

Column 3: limiting AB magnitude of the image estimated as the magnitude of a SNR $=5$ detection (see Section 2.1 for details on the flux measurement).

Column 4: median FWHM of the PSF in arcseconds measured in a large number of stars (see Section 5.4 of Paper I for details on the stellarity criteria).

Column 5: galactic extinction estimated from the Schlegel et al. (1998) maps and assuming and average value of $E(B-V)=0.004$

Column 6: zero-point corrections applied to the photometric bands, computed by comparing observed and synthetic magnitudes for spectroscopic galaxies (see Section 3.3).

filter transmission profiles for each band, and Table 1 presents the effective filter wavelengths, the survey depths, and image quality achieved in each band, and the (small) zero-point recalibrations (Section 3.3).

The photometric coverage of the EGS is largely inhomogeneous, with each band covering a different portion of the IRAC mosaic (Davis et al. 2007). Fortunately, there is a natural way to divide the field into two smaller sub-regions. The main region, defined by the overlapping area of the CFHTLS and IRAC frames $\left(0.35 \mathrm{deg}^{2}\right)$, presents the densest coverage ( 19 bands, including GALEX, Hubble Space Telescope (HST), and MOIRCS). This region is essentially a field with the side edges following the contours of the IRAC image, i.e., inclined by $50^{\circ}$ east of north, and upper and lower boundaries limited by $52.16<\delta<53.20$. The bottom-right side is also restricted to $\alpha>214.04$ due to the intersection with the CFHTLS mosaic (a square field oriented north up, east left).
The $0.13 \mathrm{deg}^{2}$ outside of the main region (hereafter referred to as flanking regions) also have solid optical-to-NIR coverage. However, the overall data quality is slightly lower than in the main region. The median coverage includes only 11 bands, and for the most part lacks the deepest, highest-resolution imaging. As a result, the quality of the SED coverage in the flanking regions is significantly lower than in the main region. For these reasons we focus in this contribution on the main region.

\subsection{Multi-band Identification and Photometry}

The procedure followed to build consistent UV-to-FIR SEDs from the multiple data sets is described in detail in Paper I (see also Pérez-González et al. 2005 and Pérez-González et al. 2008, hereafter PG05 and PG08). This section summarizes the most relevant elements of the method, so that the impact of the photometric uncertainties on the parameters estimated from the SED modeling can be assessed (Sections 4 and 5).

First, multi-band identification is carried out by crosscorrelating the $3.6+4.5 \mu \mathrm{m}$ selection with all other optical/ NIR catalogs (pre-computed with SExtractor; Bertin \& Arnouts 1996) using a $2^{\prime \prime}$ search radius. The MIPS, Radio, and X-ray catalogs required a different approach. For the MIPS and radio catalogs we used a 2 .'5 and $3^{\prime \prime}$ matching radius, respectively. For the X-ray catalog we used a $1^{\prime \prime}$ or $2^{\prime \prime}$ radius depending on whether the X-ray sources were pre-identified in any other band (Laird et al. 2009). When two or more optical/NIR counterparts separated by $>1^{\prime \prime}$ (approximately half the FWHM in IRAC-3.6) are identified within the search radius, we apply a de-blending procedure to incorporate the multiple sources in the catalog (e.g., irac070100 would become irac070100_1 and irac070100_2). Roughly $10 \%$ of the IRAC sources present two or more counterparts in the ground-based images.

Once the sources are identified, the photometry was computed separately in all bands, to properly account for the significant differences in spatial resolution. The fluxes were then combined to derive the merged SED. The procedure is carried out using our custom software Rainbow based on the photometric apertures obtained from a previous SExtractor run.

For the optical and NIR bands, total fluxes were estimated using Kron (1980) elliptical apertures. The properties of the aperture are the same in all bands (although different between objects) and are defined from a reference image, which is chosen by sorting the bands according to depth and picking the first band with a counterpart positive detection. Thus, this image is usually among the deepest, and presents a spatial resolution representative of the entire data set (typically SUBARU-R or CFHTL- $i^{\prime}$ ). Nevertheless, as a precaution, we established a minimum aperture size equal to the coarsest seeing in all bands (1".5). Although the choice of reference band depends on the cross-identification, the flux is measured in all bands independently of the counterpart detection. If a source is detected by IRAC only (i.e., there is no optical/NIR reference image), we use a fixed circular aperture of minimum size. If the source is detected in just a few optical/NIR bands (e.g., it is detected in $r$ but not in $z$ ) we still use the reference aperture in the un-detected bands. In this way, we recover fluxes for very faint sources not detected by SExtractor. If the forced measurements do not return a positive flux, the background flux from the sky rms within the aperture instead. These non-detections were not used for the subsequent SED fitting procedure.

The IRAC photometry was computed using circular apertures of $2^{\prime \prime}$ radius and applying an aperture corrections estimated from empirical point-spread function (PSF) growth curves. The 
measurement is carried our simultaneously in the four IRAC channels, using the $3.6+4.5 \mu \mathrm{m}$ positions as priors for the 5.8 and $8.0 \mu \mathrm{m}$ bands, which are much less sensitive. In the case of blended IRAC sources (i.e., those with multiple optical/ NIR counterparts), we recomputed the photometry applying a deconvolution method similar to that used in Grazian et al. (2006a) or Wuyts et al. (2008), which essentially relies in using smaller 0.9 radius apertures with larger aperture corrections. Paper I describes the accuracy of the deblending technique. For the GALEX (FUV, NUV) bands we drawn the photometry from the source catalog of the public data release GR3. This is computed with aperture photometry based on SExtractor (Morrissey et al. 2007). For the IRAC sources missed in this catalog (only $\sim 8 \%$ and $25 \%$ of the IRAC catalog is detected in the FUV and NUV bands, respectively; see Table 4 of Paper I) we used the forced measurement method described above. The photometry in the MIPS $(24 \mu \mathrm{m}, 70 \mu \mathrm{m})$ bands was carried out using PSF fitting with IRAF-DAOPHOT and aperture corrections (see PG05 and PG08 for more details).

The photometric uncertainties were computed simultaneously with the flux measurement. Although the Rainbow measurements are SExtractor-based, the SExtractor photometric errors were not used, because these are often underestimated due to correlated signal in adjacent pixels (Labbé et al. 2003; Gawiser et al. 2006). Instead, we used three different approach that range from a SExtractor-like method to a procedure similar to that described in Labbé et al. (2003, i.e., measuring the sky rms in empty photometric apertures at multiple positions). The photometric uncertainty was set to the largest value thereby derived.

The final multi-wavelength catalog contains 76,493 and 112,428 sources with [3.6] $<23.75 \mathrm{mag}$ and [3.6] $<24.75 \mathrm{mag}$, respectively (these magnitude cuts correspond to the $85 \%$ and $75 \%$ completeness levels of the IRAC mosaics). Approximately $68 \%$ of the sources are located in the main region $(52,453$; $[3.6]<23.75)$. Spectroscopic redshifts have been assigned to $10 \%$ of the sample (only 120 are at $z>1.5$ ). A total of 2913 stars have been identified based on several optical/NIR color criteria (see Section 5.4 of Paper I). A source was identified as a star only if three or more criteria were satisfied. The stellarity value (as the total number of criteria satisfied) is given in Table 7 (see Section 6). The fractions of IRAC sources detected at $24 \mu \mathrm{m}$ and $70 \mu \mathrm{m}$ are $20 \%$ and $2 \%$, respectively. Finally, a total of 990 and 590 sources are detected in the X-ray and radio catalogs of Laird et al. (2009) and Ivison et al. (2007), respectively.

In the following, we analyze the SEDs and physical properties of the IRAC sources with [3.6] $<23.75$ mag (typically SNR $\gtrsim 10)$. Nevertheless, the catalog contains sources up to [3.6] $<24.75$ ( $3 \sigma$ limiting magnitude). The complete catalog is available in the online version of the journal or through our Web interface Rainbow navigator (see Paper I for more details).

\subsection{Galactic Extinction}

The EGS field lies at high galactic latitude benefiting from low extinction and low galactic/zodiacal infrared emission. We derive an average $E(B-V)=0.004$ based on the maps of Schlegel et al. (1998) based on several positions evenly spaced along the strip, centered at $\alpha=241.80, \delta=52.80$. In our analysis, a differential galactic extinction for each band is computed assuming a Cardelli et al. (1989) curve with $R=$ 3.1. These corrections, summarized in Table 1, are not included in the photometric catalog (presented in Paper I) but these are applied before applying the SED fitting procedure.

\section{SED ANALYSIS: PHOTOMETRIC REDSHIFTS}

\subsection{Rainbow Code}

We computed photometric redshifts for all IRAC sources from the multi-color catalog presented in Paper I using our own dedicated template fitting code (Rainbow software hereafter; see PG05 and PG08). The program creates a grid of redshifted galaxy templates in steps of $\delta z=0.01$ and then applies a $\chi^{2}$ minimization algorithm to find the template best fitting the multi-band photometry. Upper limit detections and fluxes with uncertainties larger than 0.5 mag are not included in the fit. The $\chi^{2}$ definition takes into account the flux uncertainties of each band, being defined as

$$
\chi^{2}=\sum_{i=0}^{N(\text { band })}\left[\frac{F_{\mathrm{obs}, i}-A \cdot F_{\mathrm{temp}, i}}{\sigma_{i}}\right]^{2},
$$

where $F_{\text {obs,i }}$ is the observed flux in the $i$ filter and $\sigma_{i}$ is its uncertainty, $F_{\text {temp,i }}$ is the flux of the redshifted template in the $i$ filter (obtained by convolving the template with the filter transmission curve). A scaling factor is applied to the input template to fit the observed photometry. This normalization parameter $\mathrm{A}$ is used to compute quantities such as the stellar masses, absolute magnitudes, or SFRs (see Sections 4.1 and 5.2).

Prior to the $\chi^{2}$ minimization procedure, the Rainbow code gets rid of deviant and redundant photometric data points. The fluxes presenting a very steep gradient with respect to the surrounding bands are flagged and removed before attempting the final fit.

By analyzing the $\chi^{2}(z)$ distribution of the best fit in the model grid, we built the redshift probability distribution function ( $z$ PDF), from which we computed the most probable redshift and $1 \sigma$ errors, $z_{\text {best }}$ and $\sigma_{z}$. The single value that minimizes $\chi^{2}(z)$ is $z_{\text {peak }}$. We found that $z_{\text {best }}$ provided the most accurate results presenting less outliers and a smaller scatter when compared with spectroscopic redshifts. The uncertainties in the photometric redshifts are used to compute the uncertainties in the stellar parameters derived from the best-fitting template.

The Rainbow code also analyzes the dust emission on sources with at least one flux measurement beyond rest-frame $8 \mu \mathrm{m}$, i.e., the MIPS 24 and $70 \mu \mathrm{m}$ bands (see Section 5.2). Figure 2 shows the combined optical and IR SED along with the estimated physical parameters for a galaxy at $z \sim 1$ as an example of the optical and IR fitting techniques described here and in Section 5.2. The best-fit optical template to the data was used to estimate the photometric redshift, stellar mass (see Sections 3 and 4), and also the rest-frame UV flux. Moreover, IR luminosities and SFRs were obtained from the best-fit IR template to the data at rest-frame $\lambda>5 \mu \mathrm{m}$ (see Section 5.1).

\subsection{Stellar Population Templates}

The stellar templates used by the minimization code are extracted from a library of synthetic templates built by fitting SPS and dust emission models to a representative sample of galaxies at different redshifts. This reference sample is drawn from the GOODS-N and GOODS-S IRAC surveys and have highly reliable spectroscopically confirmed redshifts $(0<z<$ 3 ) and at least 10 measurements of the SEDs from the UV to the MIR. A detailed description on the modeling of these templates is given in PG08. Here, we briefly summarized their most relevant characteristics. 


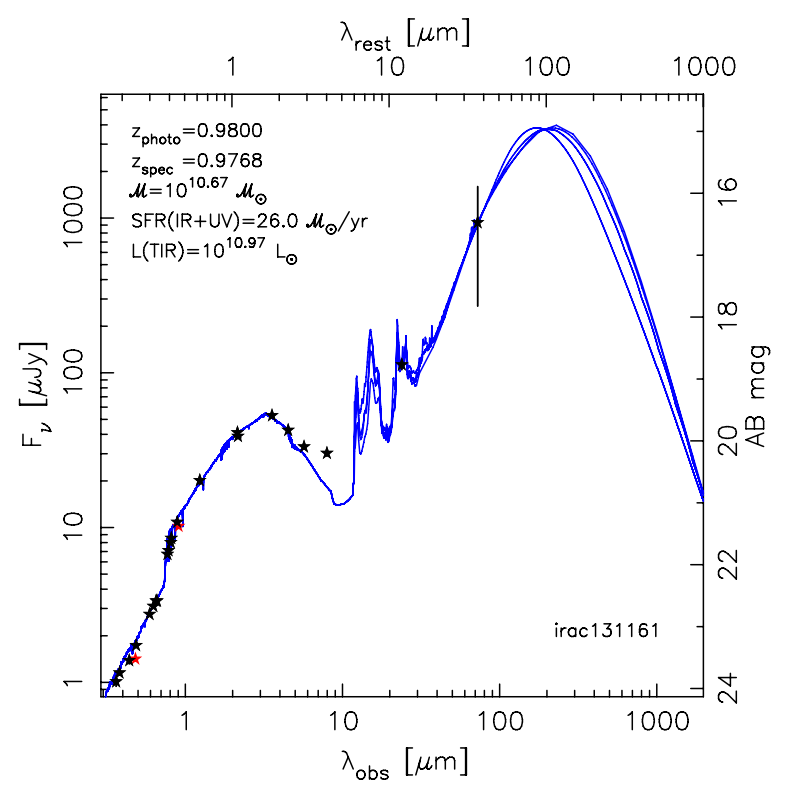

Figure 2. Example of the full Spectral Energy Distribution (SED) of a galaxy in our sample (black dots), and fit (blue lines) of the observed UV-to-MIR photometry to a set of empirical templates computed from PEGASE 2.0 models (Fioc \& Rocca-Volmerange 1997) assuming a Salpeter IMF $\left(M \in[0.1-100] M_{o}\right)$, and Calzetti et al. (2000) extinction law (see Section 3), and the FIR photometry (MIPS 24 and $70 \mu \mathrm{m}$ ) to dust emission models of Chary \& Elbaz (2001), Dale \& Helou (2002), and Rieke et al. (2009; see Section 5.1). The multiple lines in the FIR region correspond to best-fitting template from each of the dust emission models, and the average value of the three. In the upper left corner, we indicate the photometric redshift, and the stellar mass, IR-based SFR, and total IR luminosity estimated from fitting procedure.

(A color version of this figure is available in the online journal.)

The stellar emission of the reference template set was characterized using the PEGASE 2.0 models (Fioc \& RoccaVolmerange 1997) assuming a Salpeter $\operatorname{IMF}\left(M \in[0.1-100] M_{o}\right)$ and Calzetti et al. (2000) extinction law. We also considered the contribution from emission lines and the nebular continuum emitted by ionized gas. The models were obtained assuming a single population (1-POP models), characterized by an exponential star formation law. As a result, each template is characterized by four parameters in the 1-POP case, namely, the time scale $\tau$, age $t$, metallicity $z$, and dust attenuation $A(\mathrm{~V})$. The MIR/FIR region of some templates includes a contribution from a hot dust component that was computed from dust emission models using a similar procedure to that described in Section 5.1 of this paper.

Defining a representative spectral library is a critical issue for photometric redshift codes, specially when NIR-selected samples are studied (Kriek et al. 2008). The reference sample should span a wide range of redshifts and galaxy colors that probe the parameter space of the magnitude limited sample in sufficient detail. This is why we included in the template set a few $z>1.5$ galaxies which could not be fitted accurately with low $z$ templates. Furthermore, we complemented our synthetic templates with QSO and AGN empirical templates drawn from Polletta et al. (2007) that account for the galaxy population whose UV-to-NIR emission is not dominated by stars but by an AGN (see, e.g., Assef et al. 2010).

The template library contains a total of 1876 semi-empirical templates (see PG08 for more details and examples of the SEDs) spanning a wide range of colors and physical parameters. Figure 3 shows that the loci of the observed and template colors present an overall good agreement for the majority of the spectroscopic galaxies in a wide range of optical and NIR colors. The combination of colors based on the CFHTLS filters (Panels 1-4) is consistent with Figure 2 of Ilbert et al. (2006a) that presents the same colors for a sub-sample of $i$-band selected galaxies in the CFHTLS-D1 field.

On the other hand, we find small discrepancies between templates and observations in the [3.6]-[4.5] IRAC color at low redshift (Figure 3, Panel 6). This is not surprising considering that these bands are probing the rest-frame NIR (see, e.g., Huang et al. 2004; Brodwin et al. 2006 for similar examples), a wavelength region where the predictions from SPS models tend to be more uncertain (Maraston 2005). Furthermore, these differences tend to increase at $\lambda \gtrsim 3 \mu \mathrm{m}$ rest-frame, where galaxies can exhibit a significant contribution from hot dust or polycyclic aromatic hydrocarbon (PAH) emission features that are not contemplated in the optical templates and therefore require more complicated modeling procedures (Magnelli et al. 2008; Mentuch et al. 2009).

\subsection{Zero-point Corrections and Template Error Function}

An improvement introduced in the current work on EGS over the previous Rainbow photometric redshifts in GOODS$\mathrm{N}$, GOODS-S, and Lockman Hole (PG05, PG08) is the finetuning of the photometric zero points and the use of a template error function. Both procedures are based on the comparison of the observed fluxes to synthetic photometry derived from the convolution of the filter transmission curves with the best-fitting templates for the galaxies with reliable spectroscopic redshifts. As demonstrated by Brodwin et al. (2006), Ilbert et al. (2006a), and Ilbert et al. (2009), the comparison between the observed apparent magnitudes and synthetic fluxes often shows small offsets that can lead to systematic errors in the calculation of the photometric redshifts. These offsets can be the result of small systematic errors in the absolute calibration, uncertainties in the filter transmission curves, or they can be the result of intrinsic limitations of the templates in reproducing the observed SEDs (Brammer et al. 2008).

To tackle these issues and improve the photometric redshift estimation, we fit the SEDs of the galaxies with secure spectroscopic redshift to our template set fixing the photometric redshift to the spectroscopic value. Then, we compute the difference between the observed fluxes and the template fluxes for each band, and we consider this residual value as a function of the rest-frame wavelength. The left panel of Figure 4 shows the result of applying this process to the sub-sample of galaxies with spectroscopic redshift and photometric fluxes with $\mathrm{SNR}>5$ in the main region. The median of the residuals (thick green line) shows an overall good agreement between templates and observations, with an rms (thin green lines) of $\sim 2$ times the median value of the photometric uncertainty across all the wavelength range (red lines). However, significant deviations appear in the rest-frame wavelengths around $200 \mathrm{~nm}$, the 500-1000 $\mathrm{nm}$ region, and the mid-IR $(\lambda>3 \mu \mathrm{m})$.

To diminish the effect of these discrepancies, we considered two corrections: (1) we applied small calibration offsets in each band based on the residuals of the comparison with synthetic magnitudes (note that these corrections refer to observed wavelengths) and (2) we used a template error function such as that introduced in Brammer et al. (2008).

Figure 5 shows the comparison between observed and synthetic magnitudes for three different $i$-bands (ACS, CFHTLS, and MMT; left panel) and the $u *, z^{\prime}, J$, and $K$ bands (from MMT, WIRC, and MOIRCS; right panel) as a function of redshift. The values in the parenthesis quote the median correction applied to 

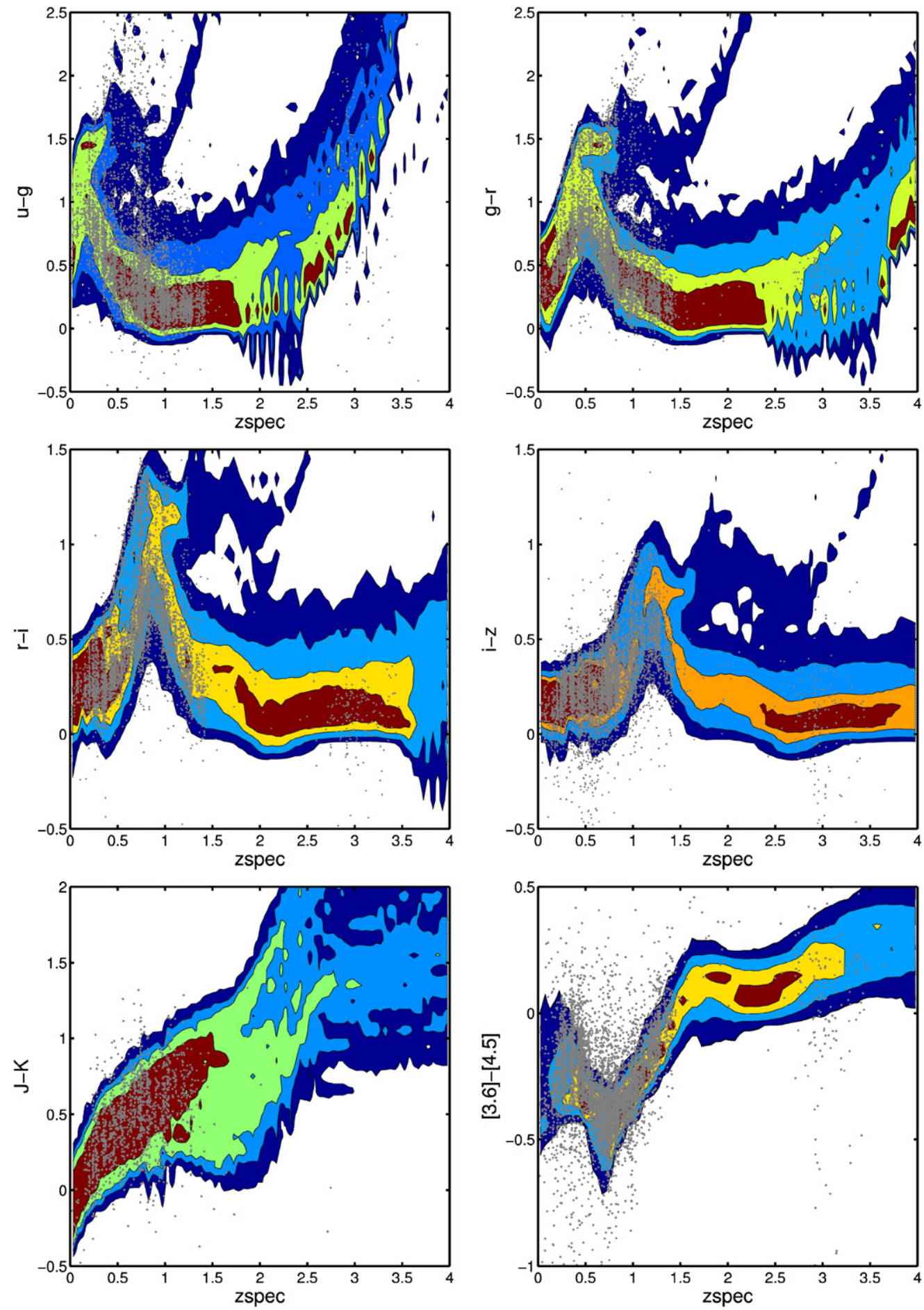

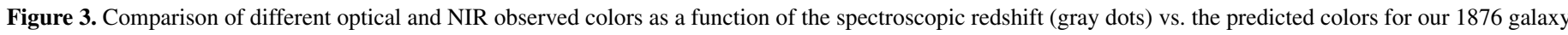
templates (density map). Each density contour contains (from inside out) $25 \%, 50 \%, 75 \%$, and $90 \%$ of the values.

each band to minimize the differences with respect to the synthetic fluxes. Note that the three $i$-bands present a similar trend at $z \gtrsim 1$, where the observations are slightly brighter than the predictions from the templates. This suggest that the feature is related to the templates and not to the absolute calibration of the bands. At $z \gtrsim 1$ the $i$-band $\left(\lambda_{\text {eff }} \sim 800 \mathrm{~nm}\right)$ probes rest-frame wavelengths around $\sim 300-400 \mathrm{~nm}$, where the overall quality of the fit to templates is reduced.

The overall shape of the residual distribution, shown in the left panel of Figure 4, is very effective for identifying systematic deviations in the templates. This is because small zero-point errors in any of the individual bands are smoothed over the restframe wavelength range due to the mixed contribution from multiple bands. Therefore, based on the overall scatter in the residual with respect to the median photometric errors, we can compute a template error function that parameterizes the overall uncertainties in the templates as a function of wavelength. As demonstrated by Brammer et al. (2008), this function can be efficiently used as a weight term in the $\chi^{2}$ function of the SED fitting procedure to minimize the impact of the template 

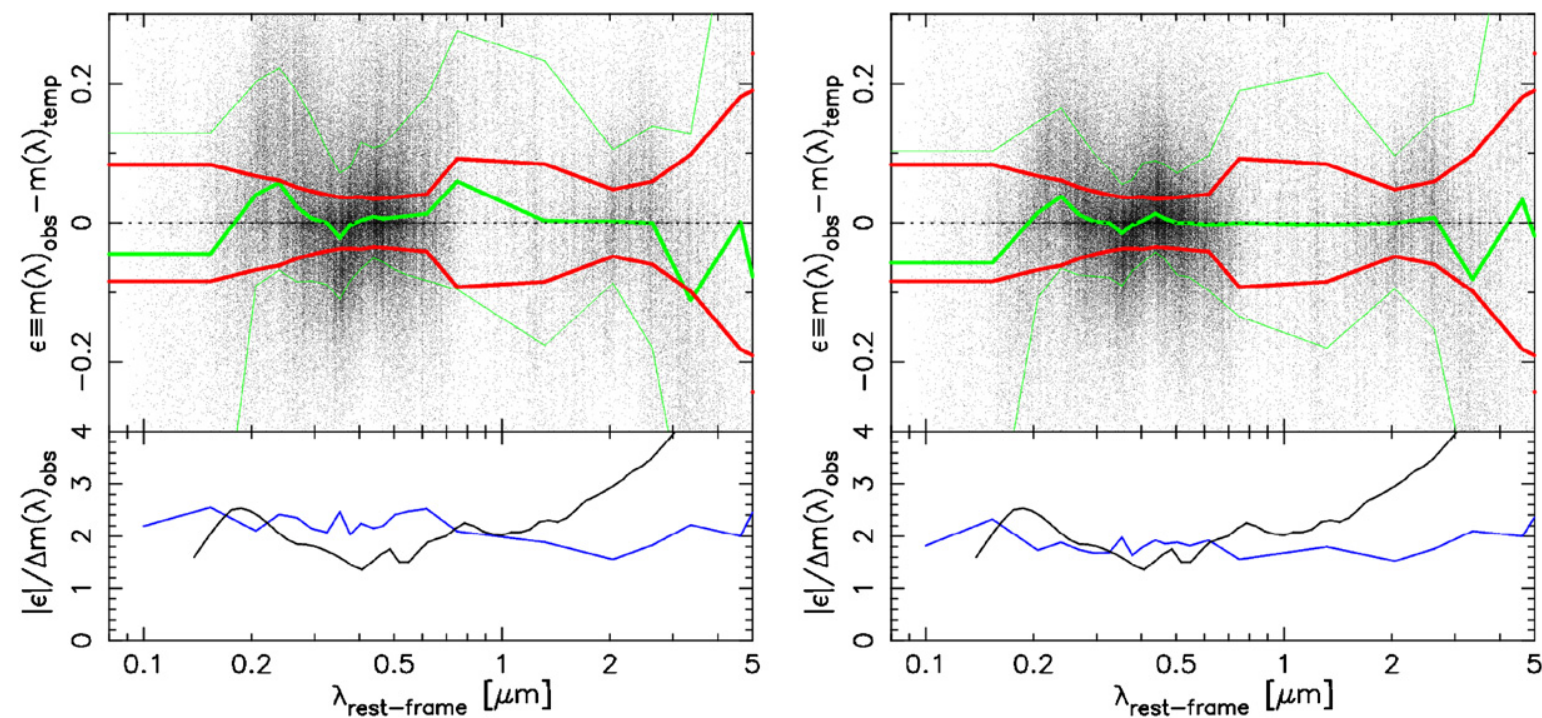

Figure 4. Top: residuals of the comparison between observed and synthetic magnitudes for a sub-sample of galaxies with spectroscopic redshift and SNR $>5$ photometry in the main region. The residuals are shifted into rest-frame wavelengths based on the effective wavelength of the filters and the redshift. The figure on the left shows the raw residuals before applying the zero-point corrections nor the template error function to the fitting procedure. The figure on the right shows the final result of the iterative process to compute the zero-point corrections and the template error function. The thick green line depicts the median value of the residuals per redshift bin. The upper and lower red lines indicate the median value of the photometric error at each redshift. The upper and lower thin green lines encompasses $68 \%(1 \sigma)$ of the residual distribution around the median value. Bottom: the blue line depicts the median absolute value of the residuals in the top panel divided by the photometric error and by 0.67 to scale the median (50\%) to a $1 \sigma(68 \%)$ confidence interval. The black line shows the template error function of Brammer et al. (2008) divided by the median photometric error (adapted from Figure 3 of their paper).
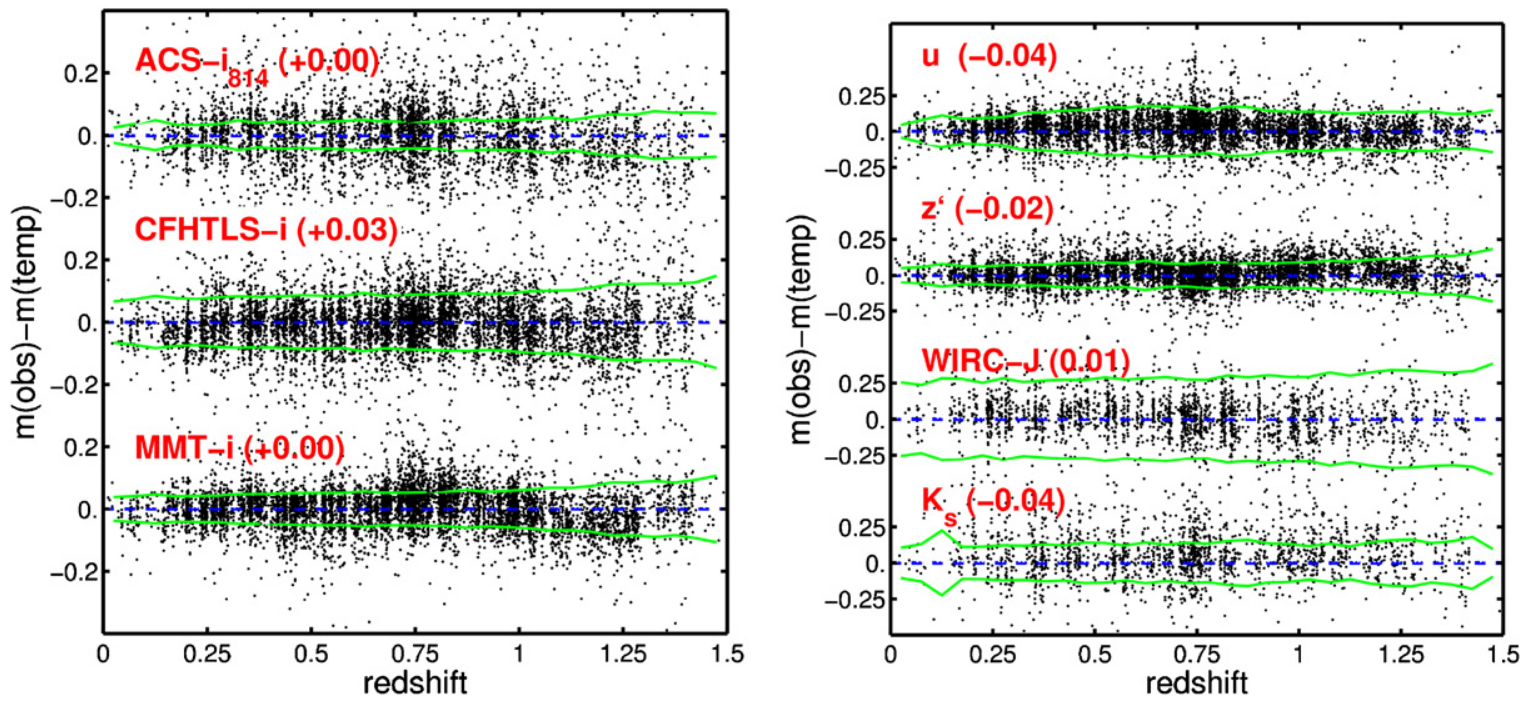

Figure 5. Left:differences between observed and synthetic magnitudes as a function of redshift in the ACS- $i_{814}$, CFHTLS- $i^{\prime}$, and MMT- $i$ bands. The values quoted in the parenthesis indicate the zero-point correction applied to these bands. The dashed blue line depicts the median difference between observed and synthetic photometry after the zero-point correction has been applied. The green lines show the median photometric uncertainty in each band as a function of redshift multiplied by a factor two. At $z \sim 1$ the residuals are dominated by a systematic offset in the templates instead of deviations in the photometric calibration. Right: Same as the left panel for the MMT- $u^{*}$, MMT- $z^{\prime}$, WIRC- $J$, and MOIRCS- $K_{s}$ bands.

uncertainties in some wavelength ranges. The bottom of the right panel of Figure 4 shows the median value of the absolute difference between observed and template fluxes divided by the photometric error and multiplied by 0.67 to scale the median $(50 \%)$ to a $1 \sigma(68 \%)$ confidence interval, as done in Brammer et al. (2008). Compared to the results of this work our combination of templates and filters present a slightly better agreement in the rest-frame UV and NIR (between 1 and $2 \mu \mathrm{m}$ ), probably as a result of our larger template set, which present more diversity in their spectral shapes.

In principle, the zero-point corrections and the effects of the template error function produce similar effects. Moreover, the re-calibration of adjacent (sometimes very similar) bands tend to modify the residual of both fits. Therefore, in order to obtain consistent results, both the template error function and the zero-point corrections are computed iteratively repeating the fitting process until we obtain variations smaller than $1 \%-2 \%$ (typically after a couple of iterations). The zero-point offsets are summarized in Table 1 . Virtually all of the corrections are smaller than $0.1 \mathrm{mag}$, and some of them are exactly zero. The final results of the procedure are shown in the right panel of Figure 4. The application of the zero-point offsets results in the flattening of the median difference between observed and template magnitudes for the whole wavelength range in our 

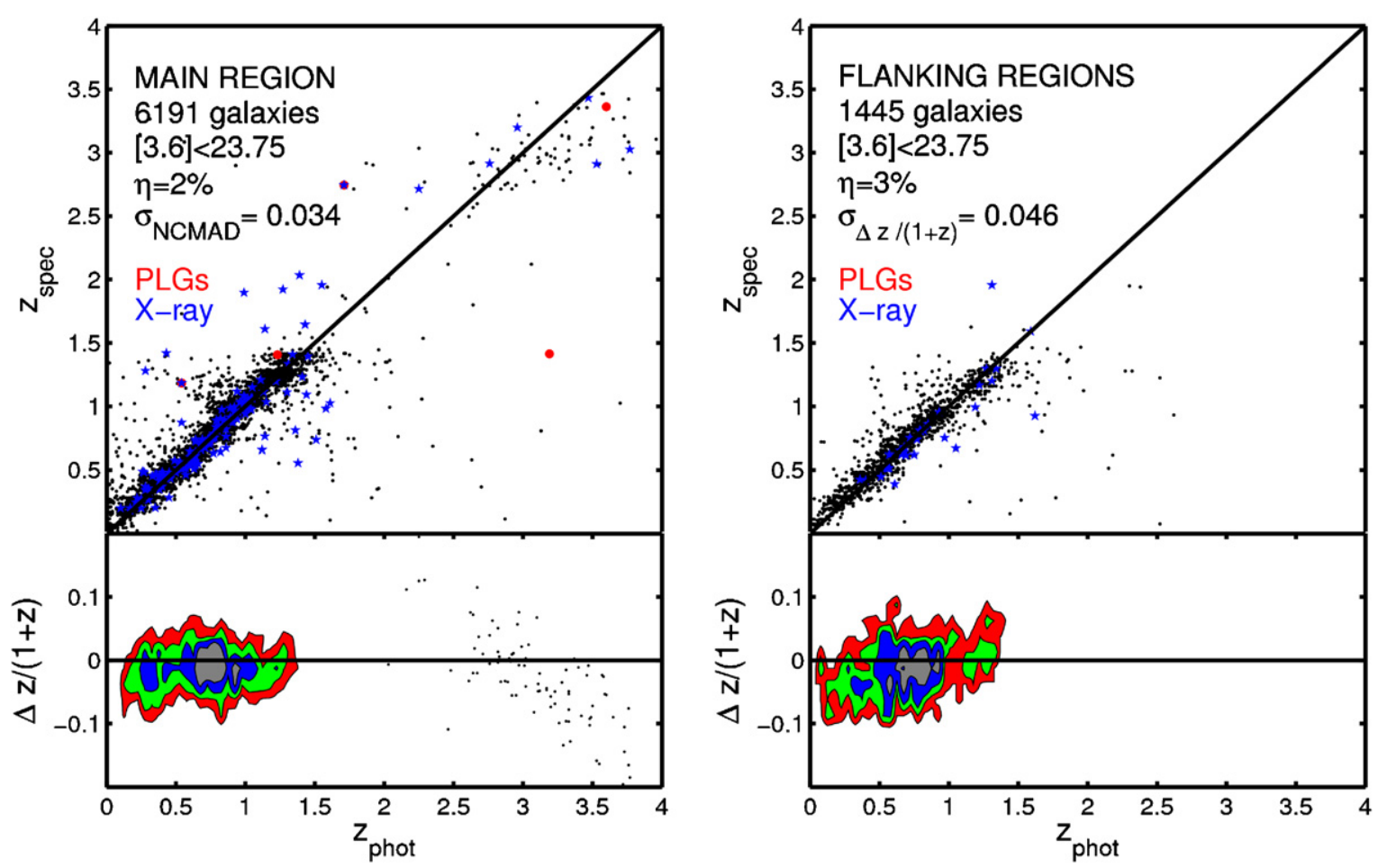

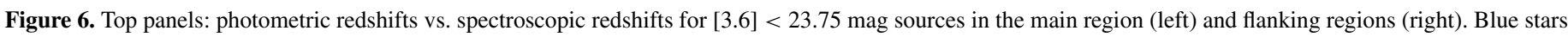

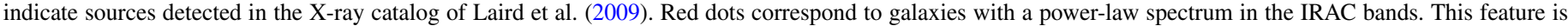

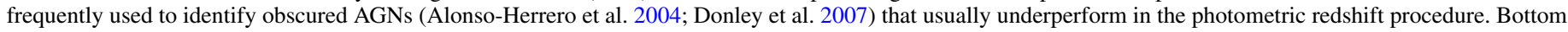

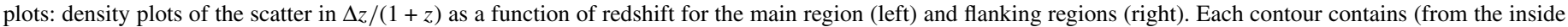
out) $25 \%, 50 \%, 75 \%$, and $90 \%$ of the spectroscopic sample, respectively.

SEDs except in two regions, one around $200 \mathrm{~nm}$ and the other at $\sim 3 \mu \mathrm{m}$.

The poor agreement at $3 \mu \mathrm{m}$ is most probably associated with limitations in the NIR-MIR range of stellar population templates and the contribution from PAH emission, which is also not properly taken into account in the SPS models nor the dust emission templates. In addition, there is a small peak/bump at $\sim 350-450 \mathrm{~nm}$, which is very similar to the feature reported by Wild et al. (2007) and Walcher et al. (2008). In these papers, they explain this effect with an excess in the strength of the Balmer break in the models by Bruzual \& Charlot (2003), relative to the observed values. The peak at $200 \mathrm{~nm}$ can be partly related with the use of a Calzetti et al. (2000) extinction curve in the modeling of the galaxy templates. This parameterization lacks the silicate absorption at $2175 \AA$, which appears in other extinction curves, such as that of Cardelli et al. (1989), which has been claimed to be produced by PAHs. The presence of this absorption bump has been reported on some studies of high- $z$ galaxies (Noll et al. 2007; Noterdaeme et al. 2009).

As an additional check of the accuracy of the method we compare our photometry against the fluxes of a control sample of 2300 bright unsaturated stars in common with the SDSS. In particular, we restrict the comparison to relatively blue sources ( $u^{\prime}-g=1.2$, in the MMT bands) in order to avoid large color corrections in the filter transformations. These color terms were computed by convolving the filter transmissions with the spectra of F, G, and K class stars (Kurucz 1992), which makes up for most of our sample of stars. The transformation with respect to the MMT bands, which present a filter system similar to that of SDSS, are

$$
\begin{aligned}
& u_{\mathrm{MMT}}=u_{\mathrm{SDSS}}-0.095 \cdot[u-g]_{\mathrm{SDSS}}+0.070 \\
& g_{\mathrm{MMT}}=g_{\mathrm{SDSS}}-0.063 \cdot[g-i]_{\mathrm{SDSS}}
\end{aligned}
$$

$$
\begin{aligned}
i_{\mathrm{MMT}} & =i_{\mathrm{SDSS}}-0.203 \cdot[i-z]_{\mathrm{SDSS}}-0.002 \\
z_{\mathrm{MMT}} & =z_{\mathrm{SDSS}}-0.087 \cdot[i-z]_{\mathrm{SDSS}}-0.002
\end{aligned}
$$

After applying these corrections, we find zero-point offsets of $\Delta u^{\prime}=-0.05, \Delta g=-0.10, \Delta i=-0.01$, and $\Delta z=$ -0.09 with respect to the SDSS. The values are roughly consistent with our previous results based on galaxy templates. Only the $u^{\prime}$ and $z$ bands present slightly lower values of the correction. These could be an additional effect of the template uncertainties (at 250 and $450 \mathrm{~nm}$ rest-frame), specially for the $u^{\prime}$ band. Also, it is worth noting that the zero-point offsets are estimated simultaneously and iteratively for all bands whereas the comparison to SDSS is done separately for each band.

\subsection{Photometric Redshift Accuracy}

In this section, we analyze the overall accuracy of the photometric redshifts $\left(z_{\text {phot }}\right)$ by comparing them against spectroscopic redshifts $\left(z_{\text {spec }}\right)$. In particular, we study the quality of our results as a function of the spectroscopic redshift and the observed magnitude in optical and NIR bands, and we provide specific results for different groups of galaxies such as X-ray, MIPS, or Radio sources. For the 76,936 galaxies $([3.6]<23.75)$ in the sample we identify 7,636 $(\sim 10 \%)$ spectroscopically confirmed sources from the DEEP2 catalog (mostly at $z<1$ ) and from a small sample of LBGs $(z \sim 3)$ presented in Steidel et al. (2003).

$$
\text { 3.4.1. } z_{\text {phot }} \text { versus } z_{\mathrm{spec}} \text { : DEEP2 Sample }
$$

Figure 6 shows the comparison between $z_{\text {phot }}$ and $z_{\text {spec }}$ for 6,191 and 1,445 sources with reliable spectroscopic redshift in the main and flanking regions, respectively.

Following Ilbert et al. (2006b), we quantified the redshift accuracy using the normalized median absolute deviation $\left(\sigma_{\mathrm{NMAD}}\right)$ 
Table 2

Photometric Redshift Accuracy in the Main Region [3.6] $<23.75$

\begin{tabular}{|c|c|c|c|c|c|c|c|c|c|c|}
\hline \multirow[b]{2}{*}{$\begin{array}{l}\text { Redshift } \\
\text { (1) }\end{array}$} & \multirow[b]{2}{*}{$\begin{array}{l}\text { No. } \\
\text { (2) }\end{array}$} & \multicolumn{4}{|c|}{ Rainbow } & \multicolumn{5}{|c|}{$E A Z Y$} \\
\hline & & $\begin{array}{c}\Delta z /(1+z) \\
\text { (3) }\end{array}$ & $\begin{array}{c}\sigma_{\mathrm{NCMAD}} \\
(4)\end{array}$ & $\begin{array}{c}\eta \\
(5)\end{array}$ & $\begin{array}{c}\Delta z_{\text {phot }} \\
\text { (6) }\end{array}$ & $\begin{array}{c}\Delta z /(1+z) \\
\text { (7) }\end{array}$ & $\begin{array}{c}\sigma_{\text {NCMAD }} \\
(8)\end{array}$ & $\begin{array}{c}\eta \\
(9)\end{array}$ & $\begin{array}{c}\Delta z_{\text {phot }} \\
(10)\end{array}$ & $\underset{(11)}{Q z}$ \\
\hline$\overline{\text { All }}$ & 6191 & 0.010 & 0.034 & $2 \%$ & 0.068 & 0.019 & 0.029 & $2 \%$ & 0.059 & $94 \%$ \\
\hline $0 .<z<0.5$ & 1637 & 0.015 & 0.040 & $2 \%$ & 0.070 & 0.019 & 0.031 & $1 \%$ & 0.049 & $97 \%$ \\
\hline $0.5<z<1.0$ & 3171 & 0.007 & 0.028 & $2 \%$ & 0.061 & 0.018 & 0.025 & $2 \%$ & 0.055 & $95 \%$ \\
\hline $1.0<z<2.5$ & 1292 & 0.017 & 0.035 & $5 \%$ & 0.083 & 0.021 & 0.032 & $4 \%$ & 0.078 & $89 \%$ \\
\hline$z>2.5$ (LBGs) & 91 & -0.023 & 0.063 & $10 \%$ & 0.110 & -0.014 & 0.043 & $15 \%$ & 0.107 & $42 \%$ \\
\hline$z>2.5($ LBGs $[3.6]<24.75)$ & 147 & -0.027 & 0.069 & $12 \%$ & 0.105 & 0.012 & 0.060 & $23 \%$ & 0.119 & $33 \%$ \\
\hline X-ray & 142 & 0.003 & 0.038 & $10 \%$ & 0.070 & 0.008 & 0.034 & $10 \%$ & 0.057 & $82 \%$ \\
\hline PLGs & 8 & 0.031 & 0.142 & $50 \%$ & 0.092 & 0.018 & 0.094 & $25 \%$ & 0.108 & $50 \%$ \\
\hline MIPS-24 $\mu \mathrm{m}$ & 1955 & 0.010 & 0.033 & $3 \%$ & 0.068 & 0.023 & 0.026 & $3 \%$ & 0.055 & $94 \%$ \\
\hline MIPS-70 $\mu \mathrm{m}$ & 262 & 0.015 & 0.045 & $1 \%$ & 0.071 & 0.031 & 0.028 & $2 \%$ & 0.050 & $95 \%$ \\
\hline Radio & 85 & -0.001 & 0.052 & $5 \%$ & 0.066 & 0.017 & 0.032 & $2 \%$ & 0.048 & $92 \%$ \\
\hline
\end{tabular}

Notes. Photometric redshift quality with the estimates with Rainbow and EAZY (see Section 3.5.2).

(1) Spectroscopic redshift range.

(2) Number of sources in the redshift bin.

$(3,7)$ Median systematic deviation in $\Delta z /(1+z) ; \Delta z=z_{\text {phot }}-z_{\text {spec }}$.

$(4,8)$ Normalized median absolute deviation.

$(5,9)$ Percentage of catastrophic outliers $(|\Delta z| /(1+z)>0.20)$.

$(6,10) 68 \%$ confidence interval in the $z \mathrm{PDF}$ around the most probable $z_{\text {phot }}$.

(11) Percentage of the sources with $Q z \leqslant 1$ in $E A Z Y$ (high quality flag according to Brammer et al. 2008).

Table 3

Photometric Redshift Quality in the Flanking Regions [3.6] $<23.75$

\begin{tabular}{|c|c|c|c|c|c|c|c|c|c|c|}
\hline \multirow[b]{2}{*}{$\begin{array}{c}\text { Redshift } \\
\text { (1) }\end{array}$} & \multirow[b]{2}{*}{$\begin{array}{l}\text { No. } \\
(2)\end{array}$} & \multicolumn{4}{|c|}{ Rainbow } & \multicolumn{5}{|c|}{$E A Z Y$} \\
\hline & & $\begin{array}{c}\Delta z /(1+z) \\
(3)\end{array}$ & $\begin{array}{c}\sigma_{\mathrm{NCMAD}} \\
(4) \\
\end{array}$ & $\begin{array}{c}\eta \\
(5) \\
\end{array}$ & $\begin{array}{c}\Delta z_{\text {phot }} \\
\text { (6) }\end{array}$ & $\begin{array}{c}\Delta z /(1+z) \\
(7)\end{array}$ & $\begin{array}{c}\sigma_{\text {NCMAD }} \\
(8) \\
\end{array}$ & $\begin{array}{c}\eta \\
(9) \\
\end{array}$ & $\begin{array}{c}\Delta z_{\text {phot }} \\
(10) \\
\end{array}$ & $\begin{array}{c}Q z \\
(11) \\
\end{array}$ \\
\hline All & 1445 & 0.013 & 0.046 & $3 \%$ & 0.065 & 0.027 & 0.050 & $4 \%$ & 0.073 & $87 \%$ \\
\hline $0 .<z<0.5$ & 373 & 0.021 & 0.058 & $2 \%$ & 0.065 & 0.037 & 0.058 & $5 \%$ & 0.052 & $83 \%$ \\
\hline $0.5<z<1.0$ & 785 & 0.015 & 0.040 & $3 \%$ & 0.059 & 0.014 & 0.069 & $8 \%$ & 0.077 & $75 \%$ \\
\hline $1.0<z<2.5$ & 274 & -0.001 & 0.058 & $8 \%$ & 0.082 & 0.009 & 0.043 & $8 \%$ & 0.079 & $70 \%$ \\
\hline X-ray & 33 & -0.019 & 0.031 & $9 \%$ & 0.057 & 0.000 & 0.035 & $9 \%$ & 0.061 & $88 \%$ \\
\hline PLGs & 0 & $\ldots$ & $\ldots$ & $\ldots$ & $\ldots$ & $\ldots$ & $\ldots$ & $\ldots$ & $\ldots$ & \\
\hline MIPS-24 $\mu \mathrm{m}$ & 416 & 0.011 & 0.046 & $4 \%$ & 0.061 & 0.028 & 0.048 & $5 \%$ & 0.068 & $88 \%$ \\
\hline MIPS-70 $\mu \mathrm{m}$ & 66 & 0.008 & 0.050 & $2 \%$ & 0.063 & 0.026 & 0.052 & $3 \%$ & 0.055 & $89 \%$ \\
\hline Radio & 13 & 0.000 & 0.055 & $8 \%$ & 0.071 & 0.035 & 0.015 & $0 \%$ & 0.060 & $85 \%$ \\
\hline
\end{tabular}

Note. Same as Table 2.

of $\Delta z=z_{\text {phot }}-z_{\mathrm{spec}}$

$$
\sigma_{\mathrm{NMAD}}=1.48 \times \operatorname{median}\left(\left|\frac{\Delta z-\operatorname{median}(\Delta z)}{1+z_{\mathrm{spec}}}\right|\right) \text {. }
$$

This quantity is equal to the rms for a Gaussian distribution and it is less sensitive to the outliers than the usual rms divided by $(1+z)$ (Ilbert et al. 2006b). We define $\eta$ as the fraction of catastrophic outliers (those sources having $|\Delta z| /(1+z)>0.20)$.

Tables 2 and 3 summarize the quality of $z_{\text {phot }}$ as a function of redshift in the main and flanking regions. The overall scatter and median systematic deviation are $\sigma_{\mathrm{NMAD}}=0.034$ and 0.046 , and $\Delta z /(1+z)=0.010$ and 0.013 for each region, respectively. As expected, the rms in the flanking regions, where the overall photometric quality is slightly lower, is higher $(\sim 20 \%)$ than in the main region. Nonetheless, the outlier fraction is only $1 \%$ worse.

The bottom panels of Figure 6 show the density plot of $\Delta z /(1+z)$ as a function of redshift. The subset of LBGs at $z_{\text {spec }}>2.5$ are shown as dots. These sources are explicitly discussed in the following section. The scatter distribution indicates that the accuracy of $z_{\text {phot }}$ does not depend strongly on the redshift up to the limit of the DEEP2 sample. The systematics in both regions are fairly similar presenting a minimum scatter at $0.5<z<1$, around the peak of the $z_{\text {phot }}$ distribution (see Section 3.7), and increasing by a factor of $\sim 1.3$ at lower and higher redshifts $\left(z_{\text {spec }} \lesssim 1.5\right)$. We find that the slightly worse performance at $z<0.5$ is associated with the use of four IRAC bands in the fitting of the SEDs. Although the template error out-weights the contribution of these bands (mostly at $\lambda>3 \mu \mathrm{m}$ rest-frame; see Section 3.3) their contribution cause a broadening of the $z$ PDF that tends to increase the scatter. Nonetheless, this effect does not increase the outlier fraction at $z<0.5$, which is comparatively lower than at $1<z<1.5$, for similar values of $\sigma_{\mathrm{NMAD}}$.

We also analyze the quality of $z_{\text {phot }}$ as a function of the optical and NIR magnitudes. As the efficiency of $z_{\text {phot }}$ mostly relies 

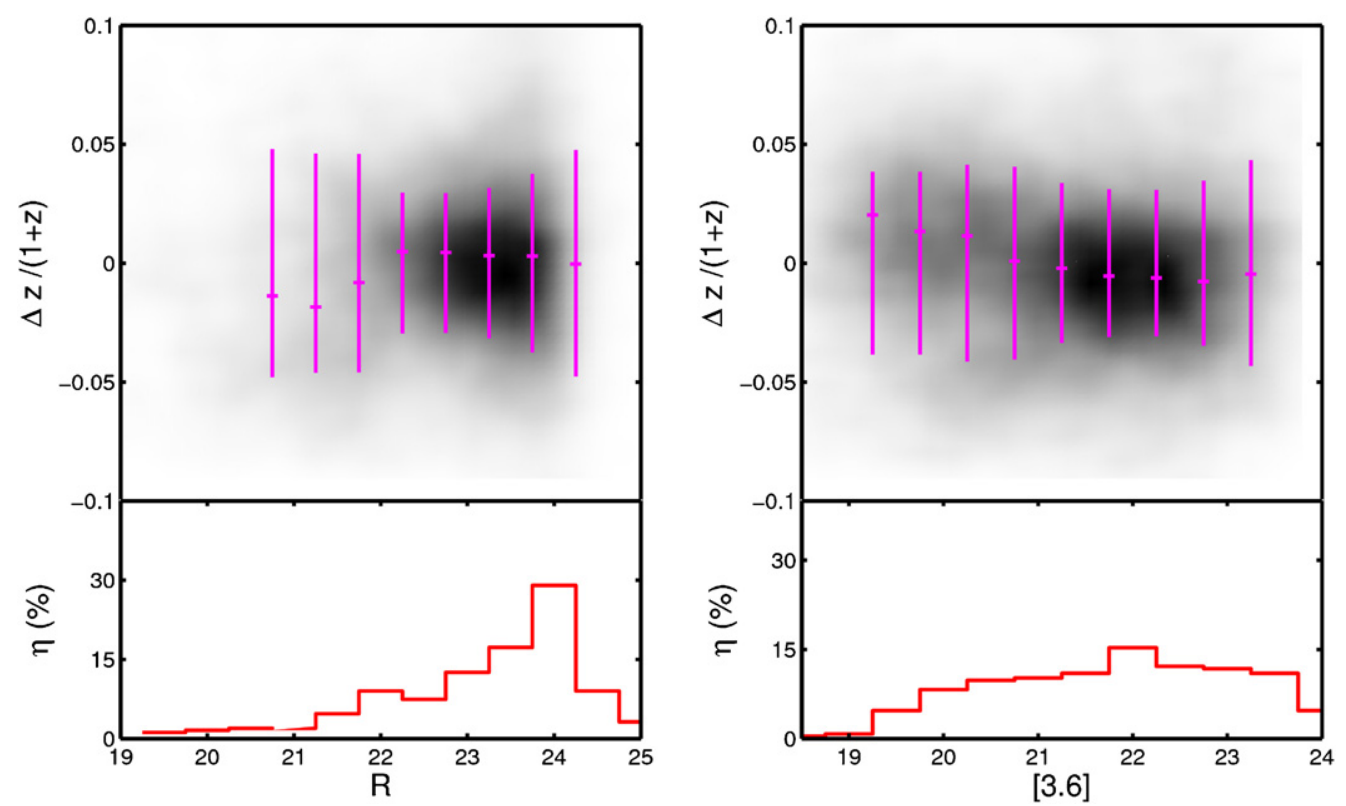

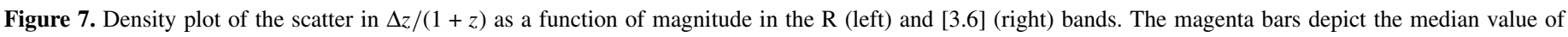

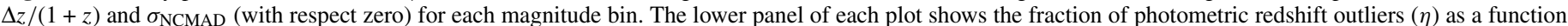
of magnitude.

(A color version of this figure is available in the online journal.)

on the detection of strong continuum features, the estimates are highly sensitive to overall consistency of the multi-band coverage. Figure 7 shows the scatter in $\Delta z /(1+z)$ as a function of the observed magnitudes in the $R$ and [3.6] bands for sources in the main region. The results in the flanking fields are similar, but with a larger scatter. We choose these bands to be representative of the brightness of the sources in the optical and NIR, and ultimately of the overall band coverage. Note that, although this is NIR-selected sample, most of the photometric coverage consist of optical bands. Thus, galaxies with faint optical magnitudes tend to present worse photometric redshifts. The magenta bars depict the median deviation and $\sigma_{\text {NCMAD }}$ per magnitude bin. We have corrected both plots by a median offset of $\Delta z /(1+z)=0.01$. In the $R$-band, the scatter increases monotonically with the optical magnitude from $\sigma_{\text {NCMAD }}=0.03-0.06$ for $R=22-25$ and $>50 \%$ of the outliers are located at $R>23.5$. The scatter is also wider at $R<22$. However, since most of these bright galaxies lie at low- $z$, this trend is essentially the same as one mentioned above for sources at $z<0.5$. Interestingly, there is weaker dependence in the scatter (and the outlier fraction) with the [3.6] mag than with the $R$-band magnitude. This is because the overall quality of the optical photometry is more relevant for constraining the shape of the SED and there is typically a wide range of optical brightnesses for any given [3.6] mag (see, e.g., Figure 6 of Paper I).

$$
\text { 3.4.2. } z_{\mathrm{phot}} \text { versus } z_{\mathrm{spec}} \text { : LBGs Sample }
$$

Given that the DEEP2 spectroscopic catalog consist mostly of low-redshift galaxies (68\% is located at $z \lesssim 0.9)$, we have included in our sample spectroscopic redshifts drawn from the LBG catalog of Steidel et al. (2003) to specifically study the accuracy of $z_{\text {phot }}$ beyond the classic spectroscopic limit. This catalog contains 334 LBGs galaxies, 193 of them with confirmed spectroscopic redshift. To check the quality of our $z_{\text {phot }}$ at $z>$ 2.5 , we first compare our results to their $z_{\text {spec }}$, and then we check that the our photometric redshift distribution for the whole LBG sample is consistent with the average redshift of this population.

We identify IRAC counterparts for 91(147) of the spectroscopic LBGs with [3.6] < $23.75 \mathrm{mag}$ (24.75). The rest were missed mainly because they lie out of the observed area in the IRAC survey; only 10 galaxies were lost due to their faintness in the IRAC bands. Note that, although these LBGs are relatively bright in the optical $(R<25.5 \mathrm{mag})$, most of them are intrinsically faint in the IRAC bands, $\sim 50 \%$ and $20 \%$ are fainter than [3.6] = $23.75 \mathrm{mag}$ and $24.75 \mathrm{mag}$, respectively. In general, LBGs are known to span a wide range of IRAC magnitudes (Huang et al. 2005; Rigopoulou et al. 2006), and they exhibit a clear dichotomy in the $R-[3.6]$ color, with red $(R-[3.6]>1.5)$ sources showing brighter IRAC magnitudes than blue sources (Magdis et al. 2008). We find that the median magnitudes and colors for the LBGs in our sample are [3.6] $=22.74, R-[3.6]=$ 2.06 and $[3.6]=23.80, R-[3.6]=0.88$ for red and blue galaxies, respectively, in good agreement with the values of Magdis et al. (2008) for a large sample of LBGs also drawn from the LBG catalog of Steidel et al. (2003).

The quality of $z_{\text {phot }}$ for the spectroscopic LBGs is summarized in Table 2. For the galaxies with [3.6] $<23.75$, both the scatter and the outlier fraction $\left(\sigma_{\mathrm{NCMAD}}=0.063, \eta=10 \%\right)$ are slightly worse than the median of the sample, as expected by their intrinsic faintness in several optical and NIR bands. Nonetheless, the statistics are similar to the results of other authors at high redshift (e.g., Wuyts et al. 2008) indicating that our $z_{\text {phot }}$ still provide reasonably consistent values beyond $z>$ 1.5. If we also consider the faintest sources ([3.6] $<24.75$ ), the statistics do not degrade much $\left(\sigma_{\mathrm{NCMAD}}=0.069\right)$, even though we are including $60 \%$ more sources. We have visually inspected the outliers and at least four of them present flux contamination from close-by sources and another three are strong AGNs detected in the X-rays. The rest of them present a high- $z$ solution in the $z \mathrm{PDF}$, but the flux at [5.8] and [8.0] is too faint to reliably identify the rapid decline of the stellar 


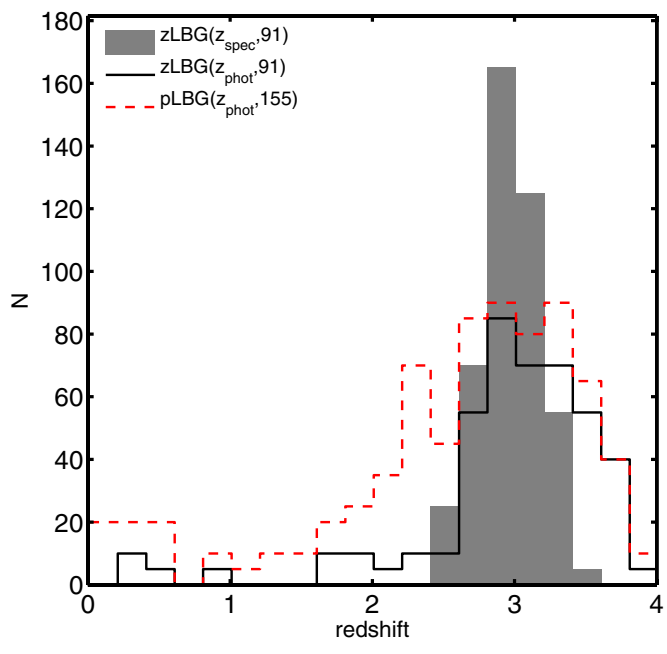

Figure 8. Photometric redshift distribution of the photometric (155, red dashed line) and spectroscopically confirmed (91, black line) LBGs (pLBG, zLBG) with [3.6] $<23.75$ in common with the sample of Steidel et al. (2003). The spectroscopic redshift distribution for the zLBG is shown as a filled gray histogram for comparison.

(A color version of this figure is available in the online journal.)

component at $\lambda>1.6 \mu \mathrm{m}$, which results on favoring the lowredshift solution.

We also compared the photometric redshift distribution of the 155 galaxies with [3.6] $<23.75$ identified in the whole LBG catalog. Figure 8 shows the redshift distribution of the photometric and spectroscopic LBGs in our sample. The median value and quartiles for the photometric LBGs with [3.6] $<23.75$ is $\tilde{z}_{\text {phot }}=2.8 \pm_{0.6}^{0.4}$ consistent with the median redshift of the spectroscopic sample $(\tilde{z}=2.95)$ and with the typical width of the redshift distribution for the LBG criteria $(\tilde{z}=3.0 \pm 0.3$; Steidel et al. 2004; Reddy et al. 2005). About $14 \%$ of these sources lie at redshift $z<1.5$, similarly to the outlier fraction of the spectroscopic sample.

\subsection{3. $z_{\text {phot }}$ versus $z_{\text {spec }}: X$-ray, Power Law, MIPS, and Radio Galaxies}

We analyze in detail the quality of the $z_{\text {phot }}$ for samples of galaxies that are known to present particularly different SEDs from the majority of the templates (e.g., X-ray or AGNs), which could cause a degradation of the redshift estimate. These sources are shown with different makers and colors in Figure 6 and their $z_{\text {phot }}$ statistics are summarized in Tables 2 and 3.

The blue stars show galaxies identified in the X-Ray Chandra/ACIS catalog in EGS (Laird et al. 2009), probably indicating the presence of an AGN. The SED of these sources is likely affected by the AGN emission, which in principle should decrease the efficiency of the template fitting procedure. In spite of showing a larger outlier fraction (particularly at $z>1.5$ ), the $z_{\text {phot }}$ for X-ray sources are quite accurate, with a scatter similar to that of the full sample.

The red dots in Figure 6 depict galaxies satisfying the powerlaw criteria (PLG) commonly used to identify obscured AGNs (Alonso-Herrero et al. 2004; Donley et al. 2007), a good fraction of them being undetected in the X-rays. We find a surface density of $0.26 \mathrm{arcmin}^{-2}$ for PLGs, in good agreement with the $0.22 \operatorname{arcmin}^{-2}$ given in Donley et al. (2007, we apply a similar criteria restricted to $P_{\chi}>0.1$ and a slope $\alpha<$ $-0.5)$. However, less than $2 \%$ of these sources present a spectroscopic redshift. Comparatively, PLGs present a lower accuracy and higher outlier fraction than the X-ray sources as a consequence of having their SED more contaminated by the AGN emission.

Similarly to the PLG, we find that sources with red colors in the IRAC bands $\left(f_{[3.6]}<f_{[4.5]}<f_{[5.8]}<f_{[8.0]}\right)$, but not strictly satisfying the PLG criteria, make up for up to $15 \%$ of the total outliers. This is not surprising given that, for the typical galaxy at $z \leqslant 2$, the presence of the stellar bump (at $1.6 \mu \mathrm{m}$ ) causes the flux in the last two IRAC bands to be lower than in the previous two. Thus, for these sources, the code will try to assign incorrect high-redshift values of $z_{\text {phot }}$.

Tables 2 and 3 also quote numbers and $z_{\text {phot }}$ statistics for the spectroscopic sub-samples of galaxies detected in MIPS $24 \mu \mathrm{m}(f(24)>60 \mu \mathrm{Jy})$, MIPS $70 \mu \mathrm{m}(f(70)>3.5 \mathrm{mJy})$, and in the catalog of Radio sources of Ivison et al. (2007). The latter present a slightly worse accuracy than the median of the sample, whereas the MIPS detected galaxies present essentially the same quality as the rest of the spectroscopic galaxies. This indicates that for most of them the IR emission does not contribute significantly to the NIR-MIR region fitted with the optical templates.

\subsubsection{Error Analysis}

The $1 \sigma$ uncertainty of the photometric redshifts, $\Delta z_{\text {phot }}$, is computed from the $z \mathrm{PDF}$ as the semi-width of the redshift range corresponding to a $68 \%$ confidence interval around the probability peak. This value allows to provide an estimate of the accuracy for sources without a spectroscopic redshift, which are $>90 \%$ of the sample.

Tables 2 and 3 quote the values of $\Delta z_{\text {phot }}$ as a function of redshift in the main and flanking regions. Based on these results, we find that $62 \%$ (approximately $1 \sigma$ ) of the galaxies present values of $\Delta z_{\text {phot }}<|\Delta z|$. The median value and quartiles of $\Delta z_{\text {phot }} /(1+z)=0.036_{0.021}^{0.056}$ in the main region are consistent with the statistics for $\sigma_{\text {NCMAD }}$ and also with $|\Delta z| /(1+z)=0.027_{0.013}^{0.050}$. A similar agreement is found for the sources in the flanking regions. Note that as the $\Delta z_{\text {phot }}$ is computed from the $z$ PDF its minimum value is limited by the step size of the redshift grid $(\Delta z=0.01)$, and thus it tends to present larger values than $|\Delta z|$, specially for very accurate $z_{\text {phot }}$. Therefore, it is not surprising that $\Delta z_{\text {phot }} /(1+z)$ is on average larger than all the other scatter estimates. In fact, this indicates that $\Delta z_{\text {phot }}$ provides a robust estimate of the uncertainty in $z_{\text {phot }}$, which can be underestimated if it assumed to be equal to $\sigma_{\mathrm{NCMAD}}$ (see, e.g., Cardamone et al. 2010).

In order to obtain a better characterization of the catastrophic outliers caused by a poor fit to the data, we analyze the distribution of sources as a function of the reduced $\chi^{2}$ of the SED fitting. Figure 9 shows the distribution of $-\log \left(\chi^{2}\right)$ for the full photometric sample, the spectroscopic sample, and the catastrophic outliers. Approximately $83 \%$ and $94 \%$ of the galaxies in the photometric and spectroscopic sample present values of $\chi^{2}$ lower than the median of the outlier distribution $\left(-\log \left(\chi^{2}\right)<-0.6\right)$, i.e., half of the outliers are located within the $\sim 20 \%$ and $5 \%$ of the sources in the photometric and spectroscopic samples with the worse values of $\chi^{2}$.

Finally, we also find that $58 \%$ of the sources with significantly different values of $z_{\text {best }}$ and $z_{\text {peak }}\left(\left|z_{\text {best }}-z_{\text {peak }}\right| /(1+z)>0.2\right)$ are outliers. These sources account for only $1 \%$ of the spectroscopic sample, but they represent $\sim 12 \%$ of the outliers. Therefore, the difference between both values is another useful indicator of possible outliers. 


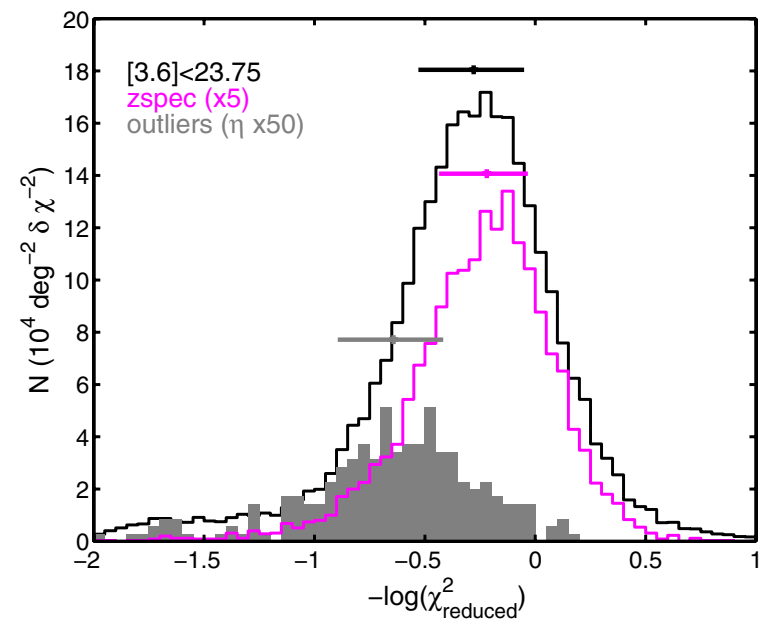

Figure 9. Distribution of the normalized $\chi^{2}$ resulting from the fit of the data to the templates during the calculation of $z_{\text {phot }}$. The black line is for the full sample $([3.6]<23.75)$, and the magenta line and gray area are for the spectroscopic sample $(\times 5)$ and photometric redshift outliers $(\eta \times 50)$, respectively. The corresponding lines depict the median value and quartiles of each distribution.

\subsection{Comparison of Photometric Redshift Catalogs}

Here, we compare the $z_{\text {phot }}$ computed with Rainbow to other previously published $z_{\text {phot }}$ catalogs and to the estimates obtained with a different code. The alternative $z_{\text {phot }}$ are also included in the our data release (see Section 6) in Table 7.

\subsubsection{Rainbow versus Ilbert et al. (2006a)}

We compare the $z_{\text {phot }}$ presented in this paper with those derived by Ilbert et al. (2006a, hereafter I06) based on optical data from the CFHTLS. These authors used an $i^{\prime}$-band selected sample with $i^{\prime}<24$ and obtained photometric redshifts for the four CFHTLS deep fields. The $z_{\text {phot }}$ were computed using the template fitting code Le phare (Arnouts \& Ilbert; e.g., Ilbert et al. 2009) for $\sim 500,000$ sources observed in five bands $u *, g^{\prime}, r^{\prime}, i^{\prime}, z^{\prime}$. Their template library is based on an upgrade of the empirical templates of Coleman et al. (1980) and Kinney et al. (1996) computed by applying zero-point corrections and interpolating between spectral types. Their $z_{\text {phot }}$ also include a Bayesian prior on the redshift distribution. The accuracy of their results for the D3 field (the EGS) is $\sigma_{\mathrm{NCMAD}}=0.035$ with $\eta=4 \%$ for sources with $i^{\prime}<24$ and $z<1.5$. More recently, Coupon et al. (2009) repeated essentially the same exercise using the latest data release of the CFHTLS T004, obtaining $z_{\text {phot }}$ of almost identical quality.

The source density in the $i^{\prime}$-band selected sample of I06 is 25,42 , and 96 sources $\operatorname{arcmin}^{-2}$ up to limiting magnitudes of $i^{\prime}=24,25$, and 26.5 (the estimated SNR $\sim 5$ level). The source density of the IRAC selected catalog is $\sim 44$ sources $\operatorname{arcmin}^{-2}$ at [3.6] $<23.75$. This means that their source density at $i^{\prime}<25$, which is essentially the spectroscopic limit $(R=25)$, is similar to ours. However, at $i^{\prime}<24$, the limiting magnitude for their best performing $z_{\text {phot }}$, the source density in $i^{\prime}$ is approximately $50 \%$ that in IRAC. At the faintest optical magnitudes, the source density in the $i^{\prime}$-band selected catalog is larger, although the quality of these $z_{\text {phot }}$ is worse than for the $i^{\prime}<24$ sample, given that many of the galaxies will also be undetected in the shallowest optical bands $\left(u^{*}, z^{\prime}\right)$.

Even presenting similar source densities, the nature of the galaxies in an $i^{\prime}$ band and an IRAC-selected samples is different, and some of the sources in one selection will be missed by the other. We find that the optically bright galaxies missed by the
Table 4

Rainbow $z_{\text {phot }}$ vs. I06 at $i^{\prime}<25$ and [3.6] $<23.75$

\begin{tabular}{|c|c|c|c|c|c|c|c|}
\hline \multirow[b]{2}{*}{$\begin{array}{c}\text { Redshift } \\
\text { (1) }\end{array}$} & \multirow[b]{2}{*}{$\begin{array}{l}\text { No. } \\
(2)\end{array}$} & \multicolumn{3}{|c|}{ Rainbow } & \multicolumn{3}{|c|}{ I06 } \\
\hline & & $\begin{array}{c}\sigma_{\text {NCMAD }} \\
\text { (3) }\end{array}$ & $\begin{array}{c}\eta \\
(4)\end{array}$ & $\begin{array}{c}R(\eta) \\
(5)\end{array}$ & $\begin{array}{c}\sigma_{\text {NCMAD }} \\
(6)\end{array}$ & $\begin{array}{c}\eta \\
(7) \\
\end{array}$ & $\begin{array}{c}R(\eta) \\
(8)\end{array}$ \\
\hline All & 5454 & 0.034 & $2 \%$ & $82 \%$ & 0.036 & $5 \%$ & $55 \%$ \\
\hline $0 .<z<0.5$ & 1444 & 0.040 & $2 \%$ & $83 \%$ & 0.032 & $5 \%$ & $43 \%$ \\
\hline $0.5<z<1.0$ & 2787 & 0.028 & $2 \%$ & $80 \%$ & 0.031 & $3 \%$ & $53 \%$ \\
\hline $1.0<z<2.5$ & 1143 & 0.035 & $4 \%$ & $80 \%$ & 0.054 & $8 \%$ & $63 \%$ \\
\hline$z>2.5(\mathrm{LBGs})$ & 80 & 0.063 & $9 \%$ & $91 \%$ & 0.345 & $46 \%$ & $42 \%$ \\
\hline
\end{tabular}

Notes.

Photometric redshift quality in the estimates with Rainbow and in I06.

(1) Spectroscopic redshift range. (2) Number of sources in the redshift bin.

$(3,6)$ Normalized median absolute deviation.

$(4,7)$ Percentage of catastrophic outliers $(|\Delta z| /(1+z)>0.20)$.

$(5,8)$ Fraction of catastrophic outliers in the other code presenting an accurate $z_{\text {phot }}$.

IRAC catalog ([3.6] > 23.75) present a median and quartile redshifts $z_{\text {phot }}=1.0_{0.6}^{1.3}$, while the infrared bright galaxies undetected in the optical $\left(i^{\prime}>26.5\right)$ present $z_{\text {phot }}=1.8_{2.3}^{1.1}$. The high- $z$ sources missed in the IR selection are typically low-mass galaxies (similar to LBGs), i.e., our catalog favors the detection of high- $z$ massive galaxies, as expected.

We cross-correlated the catalog of I06 to the IRAC selected sample using a search radius of $1^{\prime \prime} .5$. Due to small differences in the extraction of the catalogs, the comparison is restricted to a slightly smaller portion of the main region $(214.09<$ $\alpha<215.72$ and $52.20<\delta<53.16$ ). Out of the 49605 IRAC sources, $40 \%$ and $88 \%$ are detected in I06 to $i^{\prime}<24$ and 26.5, respectively. The cross-match to the DEEP2 spectroscopic redshifts contains 5454 galaxies simultaneously identified in all three catalogs $\left([3.6]<23.75, i^{\prime}<26.5\right)$. Approximately $6 \%$ of our spectroscopic subsample is missed due to a more conservative source removal around bright stars in I06.

The top panels of Figure 10 show the comparison of $z_{\text {phot }}$ versus $z_{\text {spec }}$ for the galaxies in common between the Rainbow, I06, and DEEP2 catalogs with [3.6] $<23.75$ and $i^{\prime}<25$, without any other requirement of band coverage. Table 4 summarizes accuracy of the $z_{\text {phot }}$ in Rainbow and I06 for these sources as a function of redshift. We also list the fraction of catastrophic outliers in each catalog that is recovered in the other (shown as green dots in Figure 10).

The overall scatter in I06 for the sources in common with the IRAC sample is consistent with their results for the whole D3 sample. The comparison as a function of redshift indicates that I06 estimates at lower redshift are slightly more precise, probably as a result of the template-optimization algorithm and the Bayesian prior (see Figure 6 of I06), but also because of our slightly lower performance at $z<0.5$. On the contrary, the fraction of catastrophic outliers in I06 is larger than in Rainbow for all redshifts, and particularly at $z>2.5$. Moreover, the $z_{\text {phot }}$ Rainbow is able to recover $\sim 80 \%$ of these outliers. At $z>1$, our larger band coverage, mostly in NIR bands, provides more accurate estimates. Note that the IRAC fluxes for the LBGs play a critical role in providing more accurate redshifts (and stellar parameters) for these sources.

\subsubsection{Rainbow versus $E A Z Y$}

Here, we check again the quality and overall consistency of our SEDs and $z_{\text {phot }}$ by computing an independent estimation of 

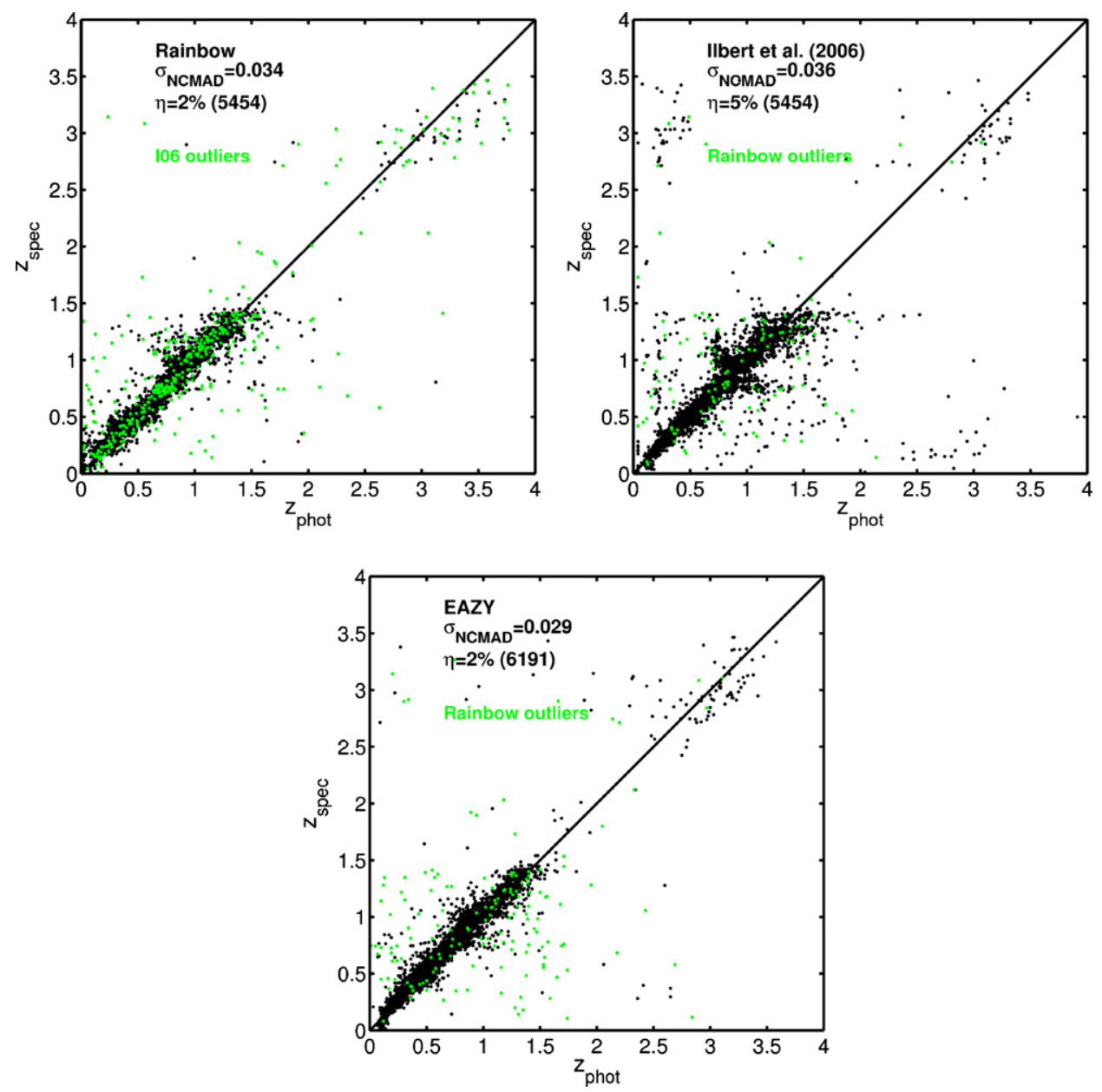

Figure 10. Top: comparison of $z_{\text {phot }}$ vs. $z_{\text {spec }}$ for the estimations presented in this paper (left) and the ones in I06 (right). The sample is drawn from the overlap region between the CFHTLS-D3 area and the IRAC mosaic (main region). Both figures contain the same 5454 sources simultaneously detected in both catalogs and in the DEEP2 sample at [3.6] $<23.75$ and $i^{\prime}<24.5$. Green points depict $z_{\text {phot }}$ in Rainbow for the outliers in I06 (left) and viceversa (right). Bottom: comparison of $z_{\text {phot }}$ computed with $E A Z Y$ vs. $z_{\text {spec }}$ for sources in the main region. This figure is equivalent to the left panel of Figure 6 for estimates based in $E A Z Y$. Green dots depict catastrophic outliers in the $z_{\text {phot }}$ estimated with Rainbow.

(A color version of this figure is available in the online journal.)

the $z_{\text {phot }}$ with a different code. A successful result using a different fitting code based on different template sets would certify that the catalog reproduces accurately the observed SEDs and is therefore suitable for galaxy population studies.

We computed alternative $z_{\text {phot }}$ using the photometric redshift code $E A Z Y$ (Brammer et al. 2008). The advantage of $E A Z Y$ is that it was conceived to provide accurate photometric redshift estimates for NIR-selected samples in absence of a representative calibration sample of spectroscopic redshifts. The code makes use of a new set of templates computed from a $K$-limited subsample of the Millennium Simulation (Springel et al. 2005; De Lucia \& Blaizot 2007) and modeled by fitting the synthetic SEDs with PEGASE models and applying an optimization algorithm. The final result is set of six templates that essentially reproduces the principal components of the catalog. Furthermore, a template error function was introduced to account for systematic differences between the observed photometry and the template photometry at different wavelengths. After trying different configurations for the input parameters, we find that the best results in the $z_{\text {phot }}-z_{\text {spec }}$ comparison are obtained using the template error function and incorporating a Bayesian prior on the redshift distribution similar to that of Benítez (2000; see Brammer et al. 2008 for more details). The use of the template error function is decisive to weight the contribution of the IRAC bands at lower redshifts as we have also verified in our own $z_{\text {phot }}$ (see Section 3.3).

In addition, we find that a critical issue to avoid a severe contamination from catastrophic $z_{\text {phot }}$ is the use of the purged photometric catalog produced by Rainbow. Prior to the fitting procedure, Rainbow carries out a first pass on the catalog where potential photometric outliers are removed. If we use the resulting catalog as input for $E A Z Y$ the outlier fraction is reduced by a factor $\sim 5$, illustrating the relevance on the photometric errors not only in the overall quality of the $z_{\text {phot }}$ but also in the catastrophic errors.

The bottom panel of Figure 10 depicts the comparison of the $z_{\text {phot }}$ with $E A Z Y$ versus spectroscopic redshifts for the galaxies in the main region (i.e., the same galaxies depicted in the left panel of Figure 6). As in the previous section green markers indicate outliers in $z_{\text {phot }}$ Rainbow. Tables 2 and 3 summarize the quality of $z_{\text {phot }}$ for Rainbow and $E A Z Y$ in different redshift bins for the main and flanking regions, respectively. The overall scatter and outlier fractions of both estimates are roughly similar, with the estimates of $E A Z Y$ presenting a slightly higher accuracy in the main region, but lower in the flanking fields. We also note that $z_{\text {phot }}$ with $E A Z Y$ perform better at $z<0.5$, being less sensitive to the mild broadening of the $z \mathrm{PDF}$ present in Rainbow. The tables also quote the $68 \%$ confidence intervals of $z_{\text {phot }}$, which 
are similar for both codes and are in good agreement with other results based on $E A Z Y$ (e.g., Cardamone et al. 2010). We show that the fraction of sources with a quality parameter $Q z \leqslant 1$ in $E A Z Y$ (good photometric redshifts; Brammer et al. 2008) is typically $>90 \%$ except for the highest redshift bin, where the photometry is more uncertain, due to the intrinsic faintness of these sources, and thus there is larger fraction of outliers. In addition, we find that roughly $40 \%-50 \%$ of the outliers in $E A Z Y$ present a poor $z_{\text {phot }}$ estimate in Rainbow and vice versa. Therefore, similarly to the galaxies with different values of $z_{\text {peak }}$ and $z_{\text {prob }}$, galaxies with significantly different estimates both catalogs are frequently $(\sim 50 \%)$ outliers.

From the good agreement between the different $z_{\text {phot }}$ estimates we conclude that the photometric catalog provides accurate SEDs suitable for studies of galaxy populations irrespective of the code used for the analysis.

\subsection{Number Densities and Redshift Distribution of NIR-selected Galaxies}

As an additional test of the accuracy of $z_{\text {phot }}$ we compare the number densities and redshift distributions of NIR colorselected populations with the results from other authors. In order to facilitate the comparison to the references, the magnitudes in this section are given in Vega system.

Given the highly non-uniform band coverage of the field, we have chosen to compute galaxy colors based on synthetic magnitudes. An advantage of this method is that synthetic photometry behaves better than directly observed values when deep data are not available in some of the required bands, allowing us to assign robust fluxes for undetected sources in the shallower bands. This is the same procedure that we used in PG08, and is similar to that presented in Grazian et al. (2007) and Quadri et al. (2007). We restrict the analysis in this section to the $0.35 \mathrm{deg}^{2}$ of the main region which count with better photometry.

For obvious reasons, the success of this method depends critically on the quality of the synthetic fluxes. In Section 3.3, we showed that these fluxes provide an accurate representation of the observed values in the magnitude range covered by the observations. The median offsets are very small and the scatter is consistent within a factor $\sim 2$ with the photometric errors at different magnitudes (see Figure 5).

In order to avoid possible selection effects, we restrict the analysis to NIR-selected galaxies which would be fully represented in the IRAC-selected sample. We selected distant red galaxies (DRGs; Franx et al. 2003) as galaxies with $[J-K]>$ 2.3, and BzK galaxies, both star forming (s-BzK) and passively evolving (p-BzK), following the equations in Daddi et al. (2004). Both criteria were proposed to target massive galaxies at $z \sim$ 2, although DRG and p-BzK are best at selecting galaxies with a significant fraction of evolved stars, whereas s-BzK select star-forming galaxies similar to those found by the low-redshift equivalent of the LBG criteria (LBG/BX; Steidel et al. 2004). For the DRG we convolved the templates with the VLT/ISAAC $J$ and $K$ filters, whereas for the BzK we used VLT/FORS B, $H S T /$ ACS $z$, and VLT/ISAAC $K$ which are the same filters used in Daddi et al. (2004).

The top left panel of Figure 11 shows the $K$-band ${ }^{6}$ number counts for the IRAC-selected catalog compared to other results from the bibliography. Our counts are in very good agreement

\footnotetext{
6 We used the following transformations when required $\Delta \mathrm{K}_{\mathrm{VEGA}-\mathrm{AB}}(\mathrm{UKIRT}, \mathrm{CFHT}, \mathrm{SOFI})=1.90,1.85,1.87$.
}

Table 5

Surface Density of DRG and BzKs

\begin{tabular}{llccc}
\hline \hline Criteria & \multicolumn{2}{c}{$K_{\text {VEGA }}<21$} & \multicolumn{2}{c}{$K_{\text {VEGA }}<20$} \\
& $\rho^{\mathrm{a}}$ & $\tilde{z}^{\mathrm{b}}$ & $\rho^{\mathrm{a}}$ & $\tilde{z}^{\mathrm{b}}$ \\
\hline DRG & 1.4 & 2.47 & 0.5 & 2.24 \\
s-BzK & 5.0 & 1.89 & 1.5 & 1.70 \\
p-BzK & 0.5 & 1.85 & 0.3 & 1.73 \\
\hline
\end{tabular}

Notes.

a Surface density of DRG and BzK in $\operatorname{arcmin}^{-2}$.

b Median photometric redshift of each sub-sample.

with the values of Quadri et al. (2007) for the MUSYC survey, and with our previous results in Barro et al. (2009) for the south region of EGS ( $\sim 30 \%$ overlap with the IRAC sample). The overall agreement with the counts of the Palomar-WIRC catalog (Conselice et al. 2008) is slightly worse. However, the complete Palomar-WIRC catalog covers a total area of $1.47 \mathrm{deg}^{2}$ out of which EGS is only a small fraction $\left(0.20 \mathrm{deg}^{2}\right)$. At $K_{\text {VEGA }} \sim$ 20 our results are also consistent with those in Hartley et al. (2008), McCracken et al. (2010), and Förster Schreiber et al. (2006), following the same trend as the latter up to $K_{\mathrm{VEGA}}=$ 21 . From this comparison, we conclude that the IRAC catalog limited to [3.6] $=23.75$ is a good proxy of a $K$-limited sample with at least $K_{\mathrm{VEGA}}<21 \mathrm{mag}$.

The top right and the bottom panels of Figure 11 shows the comparison of the number counts for DRG and BzK (restricted to $z>1.4$ ) galaxies with other values from the literature. There is good agreement within the typical scatter (0.1-0.2 dex), generally associated with cosmic variance. Our counts reproduce the most representative features of the overall distribution, namely, the plateau in DRG and p-BzK around $K_{\mathrm{VEGA}} \sim 20.5$ and the steep slope in the counts of s-BzK. We note that our s-BzK counts are slightly above those from Blanc et al. (2008) and McCracken et al. (2010) which count with very large surveyed areas $\left(0.71 \mathrm{deg}^{2}\right.$ and $2 \mathrm{deg}^{2}$, respectively). On the contrary, our results are in excellent agreement with Hartley et al. (2008, $\left.0.63 \mathrm{deg}^{2}\right)$. In McCracken et al. (2010) the authors argue that their disagreement with the counts of Hartley et al. (2008) is the result of an incorrect color correction in the filter system. However, for this work we used the exact same filters as in Daddi et al. (2004) obtaining similar results to Hartley et al. Thus, the most plausible explanation is that there is an excess of galaxies at $z \sim 1.5$ in our region.

For p-BzK, our results lie between those of Hartley et al. (2008) and McCracken et al. (2010). However, the counts of p-BzK exhibit the largest scatter of the three populations. This is not surprising given that $\mathrm{p}-\mathrm{BzK}$ target a more constrained population, prone to stronger clustering (Grazian et al. 2006b; Kajisawa et al. 2006; Hartley et al. 2008; McCracken et al. 2010) and hence significantly affected by the large-scale structure (LSS). Table 5 summarizes the accumulated surface densities of DRG and BzK galaxies up to $K_{\mathrm{VEGA}}=20$ and $K_{\mathrm{VEGA}}=$ 21 . The values are roughly consistent with the results of the studies shown in Figure 11, and with other values from the literature (0.89 DRG arcmin $^{-2}$ in Quadri et al. 2007; $3.1 \mathrm{~s}-\mathrm{BzK}$ $\operatorname{arcmin}^{-2}$ and $0.24 \mathrm{p}-\mathrm{BzK} \operatorname{arcmin}^{-2}$ in Reddy et al. 2005; 3.2 $\mathrm{s}-\mathrm{BzK} \operatorname{arcmin}^{-2}$ and $0.65 \mathrm{p}-\mathrm{BzK} \operatorname{arcmin}^{-2}$ in Grazian et al. 2007). As mentioned above, the excess of s-BzK by a factor of $\sim 1.5$ could be caused by a source overdensity in the area. Note that this excess does not necessarily affect s-BzK and p-BzK in the same manner due to the different clustering properties of each population (Hartley et al. 2008; McCracken et al. 2010; 

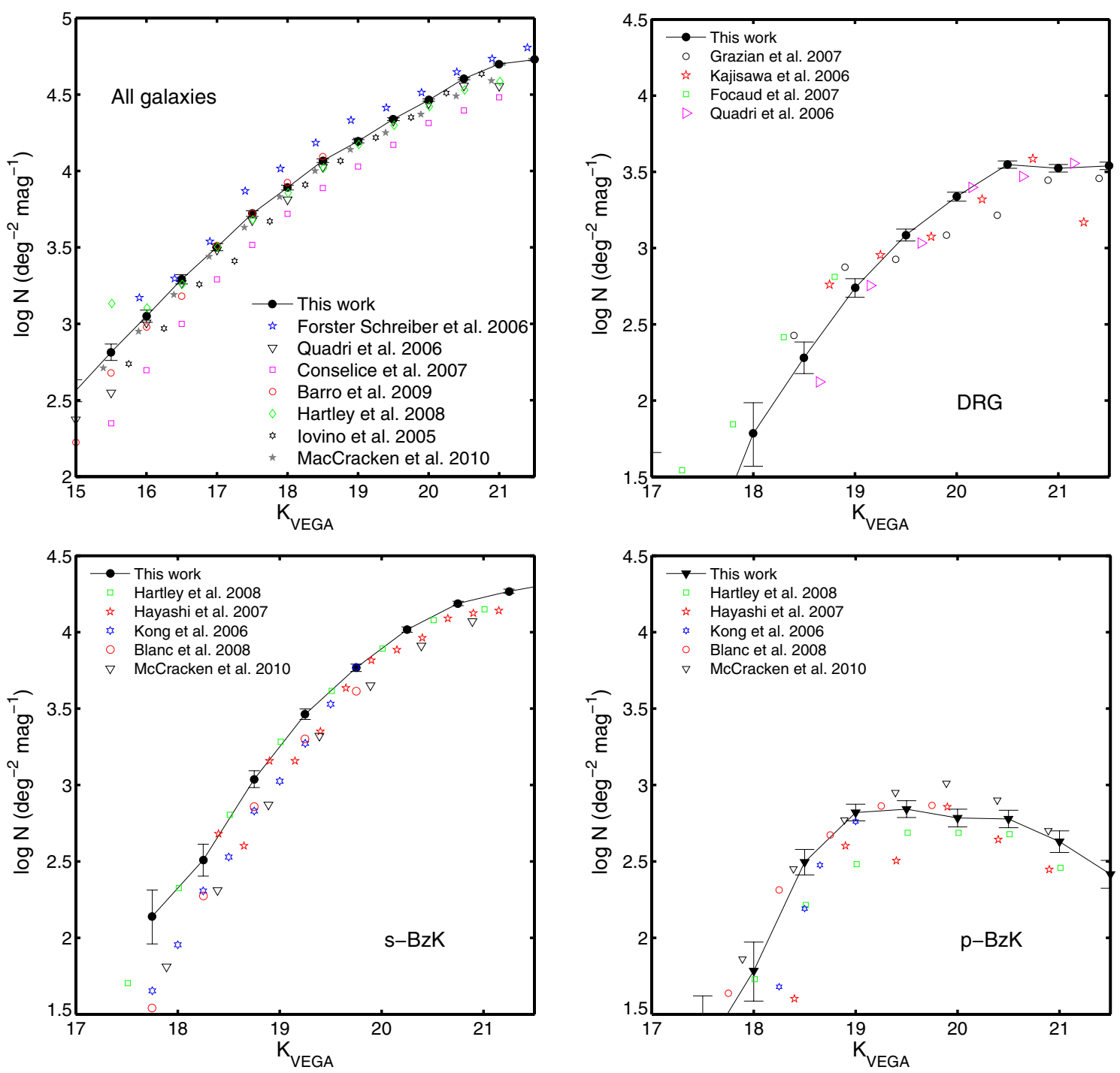

Figure 11. Galaxy number counts of our sample in the $K$-band, derived from synthetic observed magnitudes, compared to results from the literature. The black dots connected with a solid line depict the values derived in the present work. The other symbols show the results from other authors. Top left: number counts for the complete galaxy sample $([3.6]<23.75)$ in the main region. Top right: number counts for DRG. Bottom left: number counts for s-BzK galaxies. Bottom right: number counts for $\mathrm{p}$-BzK galaxies.

Hartley et al. 2010). In fact, our density of p-BzK is not among the lowest values.

Figure 12 shows the $z_{\text {phot }}$ distribution of DRG, s-BzK, and pBzK galaxies with $K_{\mathrm{VEGA}}<20$ and $K_{\mathrm{VEGA}}<21$, compared to some results from the literature. The distributions are convolved with a $\delta z=0.1$ kernel in order to account for the $z_{\text {phot }}$ uncertainties. The redshift range spanned by the different galaxy populations is in good agreement with the usual distributions observed in other studies, i.e., $z>2$ for DRG and $1.4<z<2.5$ for BzKs (Daddi et al. 2004). DRG present a secondary redshift peak around $z \sim 1$, that accounts for a significant fraction of the total population at bright $\left(K_{\mathrm{VEGA}}<20\right)$ magnitudes (as already pointed out by other authors, e.g., Quadri et al. 2007; Conselice et al. 2007). Nevertheless, our surveyed area $\left(0.35 \mathrm{deg}^{2}\right)$ is not large enough to make (bright) low- $z$ DRG the dominant fraction, as in the $0.70 \mathrm{deg}^{2}$ of the $K$-band Palomar survey where $\sim 70 \%$ of these galaxies are found at $z<1.4$ Conselice et al. (2007). As expected, s-BzK and p-BzK present almost identical redshift distributions, although the latter seems to have a more extended high-redshift tail, being also less prone to low redshift interlopers (probably as a consequence of the more restrictive color criteria). In summary, our results about the number density and redshift distribution of color-selected $z>1$ galaxy samples are consistent with previous studies (Reddy et al. 2005; Grazian et al. 2007; PG08), indicating that the photometric redshift estimates are generally robust at high redshift.

\subsection{Photometric Redshift Distribution}

Figure 13 shows the $z_{\text {phot }}$ distribution for the IRAC selected sample in the main region, limited to [3.6] $<23.75$. In addition, we also plot the redshift distributions of the galaxies detected at $24 \mu \mathrm{m}, 70 \mu \mathrm{m}$, and the sub-sample with spectroscopic redshifts. In order to derive a realistic distribution, accounting for the uncertainties in $z_{\text {phot }}$, the distribution was convolved with the typical width of the $z$ PDFs. We used a conservative upper limit of $\Delta z /(1+z)=0.07$. The shape of the distribution is consistent with that expected for a magnitude limited sample. At low redshift the number density increases as we probe larger volumes, and then an exponential decay is observed as the sources get fainter and the detection probability decreases. 


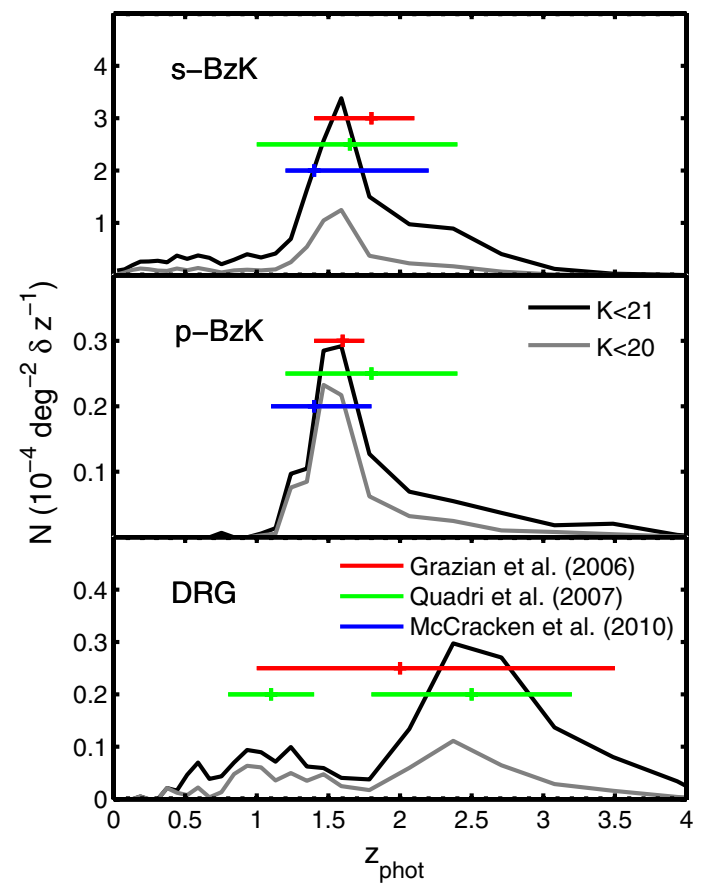

Figure 12. Photometric redshift distributions of s-BzK, p-BzK, and DRG galaxies (from top to bottom) drawn from the IRAC-selected sample with [3.6] < 23.75 . The black line and gray line show the distributions at $K_{\mathrm{VEGA}}<21$ and $K_{\text {VEGA }}<20$, respectively. Our results are compared with the median (and quartile) of the redshift distributions in Grazian et al. (2007, red), Quadri et al. (2007, green), and McCracken et al. (2010, blue; also top to bottom). The two (green) intervals in the redshift distribution of DRG indicate the median values of the distribution at redshifts lower and higher than $z=1.5$ in the work Quadri et al. (2007).

The positions of the minor prominences in the $z_{\text {phot }}$ distribution are roughly consistent with the most remarkable peaks observed in the spectroscopic redshift distribution at $z \sim 0.3$, $z \sim 0.7, z \sim 1$. The median redshift of the photometric redshift distribution is $z=1.2,75 \%$ of the sources are below $z=2.1$, and $90 \%$ below $z=2.7$. The median of the distribution is consistent with the results of Ilbert et al. (2009) in the COSMOS field for an $i^{\prime}+3.6 \mu \mathrm{m}$ selected sample $\left(i^{\prime}<25, f(3.6)>1 \mu \mathrm{Jy}\right)$. Although the IRAC S-COSMOS catalog is shallower than ours, with a $\sim 50 \%$ completeness level at $f(3.6)=1 \mu \mathrm{Jy}$, the median redshift limited to their faintest magnitude bin $\left(24.5<i^{\prime}<25\right)$, $z=1.06$, is similar to ours. Note that the small differences could arise from the presence of underlying LSS in EGS, whereas this effect is largely reduced in the COSMOS sample due the larger area of field $\left(\sim 1.73 \mathrm{deg}^{2}\right)$. Finally, the distribution is also in good agreement with our results in PG08 for the averaged redshift distribution of a combination of IRAC-selected catalogs in the HDFN, CDFS, and Lockman Hole fields. The total combined area in PG08 is approximately that of the main region of EGS, and the limiting magnitude of the catalog was slightly lower $(f(3.6)<1.6 \mu$ Jy $)$. However, the redshift distribution also peaks around $z=0.8-1$, consistently with ours.

\section{SED ANALYSIS: STELLAR MASSES}

In this section, we describe the method used to estimate stellar masses based on the SED fitting. In addition, we analyze the goodness of our stellar mass estimations quantifying the systematic and random errors linked to assumptions in the input parameters for the stellar population modeling. For the discussion in Section 4.2.1, we use only the spectroscopic sample in

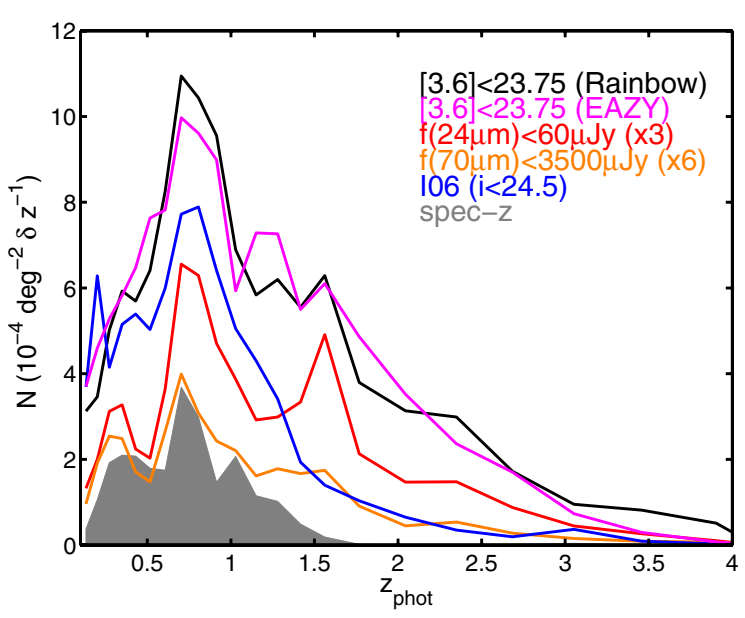

Figure 13. Photometric redshift distribution for the IRAC ([3.6] < 23.75; black), MIPS $24 \mu \mathrm{m}(\times 3$, red), MIPS $70 \mu \mathrm{m}(\times 6$, orange), and spectroscopic (gray area) samples in the main region of the EGS. The distribution of $z_{\text {phot }}$ in I06 $\left(i^{\prime}<24.5\right)$ for the overlapping area with the IRAC mosaic is shown in blue for comparison.

the main region (which count with better photometry) and we force the $z_{\text {phot }}$ to the spectroscopic value.

\subsection{Stellar Mass Estimates}

The stellar mass of each galaxy is estimated from the wavelength-averaged scale factor required to match the template monochromatic luminosities to the observed fluxes. This is possible because our templates are obtained from SPS models which are expressed in energy density per stellar mass unit. Note that the stellar mass estimate is not obtained from a single rest-frame luminosity and its corresponding mass-tolight ratio, which has been a typical procedure seen in the literature, but from the whole SED. In our method, the fit to the multi-band data implicitly constraints the mass-to-light ratio by determining the most suitable template. Then, we estimate the mass from the averaged template normalization, weighted with the photometric errors. This approach is less sensitive to the effects of the star formation history (SFH) or the photometric and template uncertainties in a single band. Objects fitted with pure AGN templates have no stellar mass estimate, as their SED is dominated by non-stellar emission. The random uncertainty of the stellar mass is estimated with a bootstrap method by randomly varying the photometric redshift and observed fluxes based on their quoted errors.

\subsection{Accuracy of the Stellar Masses}

In addition to the uncertainties inherited from the probabilistic nature of $z_{\text {phot }}$ and the intrinsic photometric errors, there is another source of systematic uncertainty associated with the assumptions in the SED modeling. Although significant effort has gone into providing accurate SPS models, key ingredients of the theoretical predictions are still poorly understood. As a result, there can be substantial differences in the physical properties estimated with many of the well-tested SPS models available in the literature. Most of these differences arise from the different parameterizations of potentially uncertain phases of the stellar evolution, such us the asymptotic giant branch (AGB) or the thermally pulsating AGB (Maraston 2005; Bruzual 2007; Kannappan \& Gawiser 2007). Another critical aspect is the choice of an IMF. Although this is essentially assumed to 

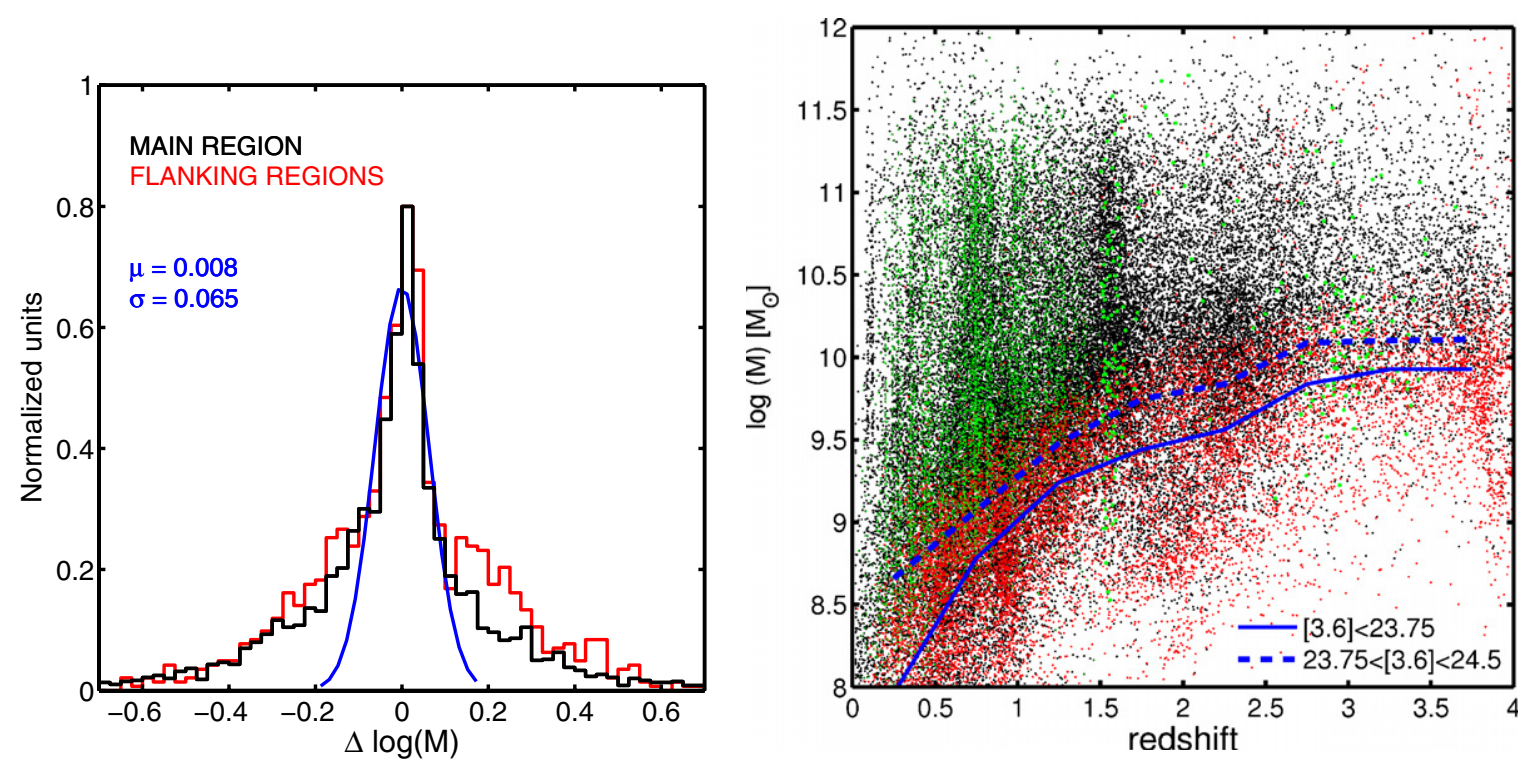

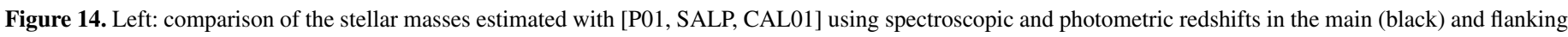

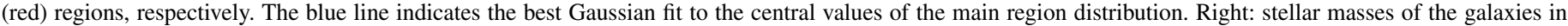

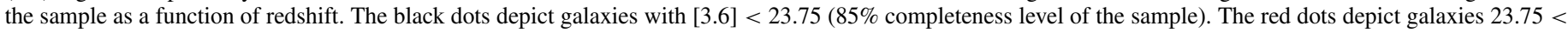

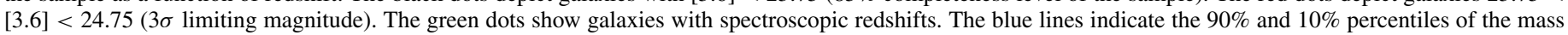
distribution as a function of redshift for the galaxies with [3.6] $<23.75$ (solid) and $23.75<[3.6]<24.75$ (dashed), respectively.

introduce a change in the overall normalization of the stellar mass, there are additional effects attached, e.g., a change in the balance between low-mass and high-mass stars varies the relative fraction of stars in different points of the isochrones. Thus, modifying the colors and $\mathrm{M} / \mathrm{L}$ of the modeled galaxies at different evolutionary stages (Maraston 1998; van Dokkum et al. 2008).

Apart from the choice of SPS models and IMF, additional effects might arise from the assumed $\mathrm{SFH}$, usually parameterized with $\tau$-models, or the choice of a dust extinction law and metallicity. As recently shown in Muzzin et al. (2009) (also Kriek et al. 2008 or Marchesini et al. 2009), due to all these effects, the physical properties of galaxies estimated from broadband photometry often presents large uncertainties (typically within a scatter of 0.2 dex for stellar masses), in addition to systematic offsets. Moreover, these uncertainties can be even larger (up to $0.6 \mathrm{dex}$ ) for particularly sensitive galaxy populations at high- $z$, such as bright red galaxies. See for example the series of paper by Conroy et al. (2009, 2010) and Conroy \& Gunn (2010) for a detailed discussion of all these issues.

Taking these considerations into account, in the following sections, we analyze the accuracy of our stellar mass estimates quantifying the uncertainty budgets associated with different effects. First, we study the effect of photometric redshift uncertainties. Then, we evaluate the impact from the choice of SPS models, IMF, and dust extinction law restricting the analysis to the spectroscopic sample. For the sake of clarity, we refer all comparisons to a default choice of SED modeling parameters (as described in 3) characterized by SPS models, IMF, and extinction law [P01, SALP, CAL01]. Finally, we verify that our stellar masses provide realistic values by comparing them to other stellar mass catalogs available in the literature.

Note that although the a priori assumptions on the SFH can also introduce systematic effects in the estimated stellar masses, an in depth analysis of these issues is clearly beyond the scope of this paper (see, e.g., Maraston et al. 2010 for a detailed discussion). Nonetheless, a comparison of the results obtained with a single exponentially declining stellar population (1-POP) and with a single population plus a second burst (2-POP) is presented in PG08 (Appendix B) along with similar tests to the ones presented in the next section.

The catalog of stellar masses presented in this paper (see Section 6) contains the different values obtained with all the modeling configurations discussed in the next sections.

\subsubsection{Effects of the Photometric Redshifts, SPS Models, $I M F$, and Extinction Law}

The left panel of Figure 14 shows the scatter in the stellar masses estimated using $z_{\text {phot }}$ and $z_{\text {spec }}$ for the 7636 spectroscopic galaxies in the main (black) and flanking regions (red). Approximately $68 \%$ and $90 \%$ of the sources are confined within an rms of 0.16, 0.34 dex and 0.20, 0.39 dex in each region, respectively. Nonetheless, the distribution shows a pronounced central peak that it is well reproduced by a Gaussian distribution (blue line) with extended wings, indicating that for the most accurate redshifts, the scatter is substantially reduced $(\sim 0.065 \mathrm{dex})$. This is in good agreement with the results of Ilbert et al. (2010, see Figure 3) scaled to the overall accuracy of our photometric redshifts, which is slightly lower. The right panel of Figure 14 shows the range of stellar masses as a function of redshift for the whole sample (black). In order to illustrate the approximate limiting stellar mass inherited from the magnitude limit ([3.6] $<23.75 ; 85 \%$ completeness), we also depict the galaxies up to the $3 \sigma$ limiting magnitude $(23.75<[3.6]$ $<24.75$, red dots). Approximately $90 \%$ of the galaxies with [3.6] $<23.75$ present $\log (M)>10 M_{\odot}$ at $z \gtrsim 2.5$ (blue line) in agreement with our results in PG08 for a similar limiting magnitude. Similarly, $\sim 10 \%$ of the faintest galaxies $(23.75<$ [3.6] < 24.75), absent in our main sample, present stellar masses larger than $\log (M)>10 M_{\odot}$ (blue dashed line). Note however that the completeness in stellar mass cannot be directly extraploted from these limits because for any given redshift, galaxies with different ages present different mass-to-light ratios. Hence, the completeness is an age (or color) dependent value. In particular, magnitude limited samples are known to be incomplete against the oldest (red) galaxies (see, e.g., Fontana 
Table 6

Comparison of Stellar Masses Computed with Different Modeling Assumptions

\begin{tabular}{lcccc}
\hline \hline \multicolumn{1}{c}{$\begin{array}{c}\text { IMFs } \\
(1)\end{array}$} & $\begin{array}{c}\text { SPS model } \\
(2)\end{array}$ & $\begin{array}{c}\text { Dust } \\
(3)\end{array}$ & $\begin{array}{c}\Delta \log (M) \\
(4)\end{array}$ & $\begin{array}{c}\Delta \log (M) \\
(5)\end{array}$ \\
\hline SALP-KROU & CB09 & CAL01 & $0.19 \pm_{0.12}^{0.07}$ & $0.19 \pm_{0.10}^{0.12}$ \\
SALP-KROU & P01 & CAL01 & $0.03 \pm_{0.17}^{0.20}$ & $0.13 \pm_{0.21}^{0.19}$ \\
KROU-CHAB & CB09 & CAL01 & $0.04 \pm_{0.09}^{0.11}$ & $0.07 \pm_{0.10}^{0.24}$ \\
\hline SPS model & IMF & Dust & $\Delta \log (M)$ & $\Delta \log (M)$ \\
\hline BC03-CB09 & CHAB & CAL01 & $0.04 \pm_{0.15}^{0.28}$ & $0.07 \pm_{0.21}^{0.30}$ \\
P01-CB09 & KROU & CAL01 & $0.15 \pm_{0.29}^{0.23}$ & $0.08 \pm_{0.27}^{0.28}$ \\
P01-M05 & KROU & CAL01 & $0.39 \pm_{0.28}^{0.36}$ & $0.30 \pm_{0.27}^{0.35}$ \\
CB09-M05 & KROU & CAL01 & $0.16 \pm_{0.28}^{0.26}$ & $0.20 \pm_{0.29}^{0.27}$ \\
\hline Dust & IMF & SPS model & $\Delta \log (M)$ & $\Delta \log (M)$ \\
\hline CAL01-CF00 & SALP & P01 & $-0.03 \pm_{0.23}^{0.20}$ & $0.00 \pm_{0.21}^{0.20}$ \\
\hline
\end{tabular}

Notes. Comparison of the stellar masses obtained under different combinations of the modeling assumptions.

(1), (2), and (3) SPS model, IMF, and dust extinction law, alternatively. The first column indicate the parameters being compared.

(4) Log of median value and quartiles of the difference for galaxies with $\log (M)<10 \mathcal{M}_{\odot}$.

(5) Same as (4) for galaxies with $\log (M)>10 \mathcal{M}_{\odot}$.

et al. 2006). A detailed analysis of the completeness limit as a function of the galaxy type will be included in a forthcoming paper.

The first test on the effect of the SED modeling assumptions consist of a comparison of the stellar masses computed with three different choices of the IMF: SALP, Kroupa (2001), and Chabrier (2003, hereafter KROU and CHAB, respectively) IMFs. The naive expectation is that the stellar masses obtained with a SALP IMF are on average larger than those obtained with the other two, as it predicts a larger number of low-mass stars. On the contrary, the IMFs of KROU and CHAB are quantitatively very similar and therefore the differences are expected to be small and mass independent. Table 6 summarizes the median value and quartiles of the comparison of stellar masses obtained with each IMF in combination with the P01 and S. Charlot \& G. Bruzual (2011, in preparation, referred as CB09) models and a CAL01 extinction law against the reference values. The top panels in Figure 15 show this comparison for SALP or KROU IMFs (left) and a KROU or CHAB IMFs (right) and the CB09 models. In both cases the difference is essentially a constant value of factor $\sim 1.6$ and 1.2 , respectively, consistently with the results of the literature (e.g., Salimbeni et al. 2009; Muzzin et al. 2009; Marchesini et al. 2009). On the contrary, the difference in the values obtained with a SALP or KROU IMFs for the P01 models is significantly smaller than that for the CB09 models, showing also a larger scatter and a dependence on the stellar mass. This effect seems to be related with a difference in the age dependency of the mass-to-light ratio for each IMF in these particular models (see, e.g., Maraston 1998; van Dokkum et al. 2008 for a description of these effects).

The second test on the modeling parameters is the comparison of stellar masses obtained with the models of P01, Bruzual \& Charlot (2003, BC03), Maraston (2005, M05), and CB09 for the same IMFs. The models of M05 were the first to account for the contribution of the TP-AGB phase in the SPSs, a consideration that is expected to lead to lower stellar masses compared to those obtained with P01 and BC03. However, this difference should be reduced in the $\mathrm{CB} 09$ models, the updated version of $\mathrm{BC} 03$, which include an improved treatment of this particular phase. The overall results of the comparison between models are also summarized in Table 6 and in the panels of Figure 16.

Interestingly, we find that the difference between [BC03, $\mathrm{CHAB}]$ and $[\mathrm{CB} 09, \mathrm{CHAB}]$ is relatively small, $\sim 0.04 \mathrm{dex}$, and mostly independent of the stellar mass. This suggests that, at least for the present sample, taking into account the TP-AGB phase does not introduce significant differences. A possible explanation could be that, since the spectroscopic sample consist mostly on $z<1$ galaxies, the available photometric coverage is not probing the rest-frame NIR with sufficient detail. Only at higher redshifts $(z \gtrsim 1)$ the IRAC bands would start probing the region of the SED that is heavily affected by the TP-AGB phases. Note also that the spectroscopic sample analyzed here might not be a critical population to constraint the effect of the TP-AGB, as, for example, the post-starburst galaxies studied in Conroy et al. (2010).

In addition, we find that the estimates with [P01, KROU] are larger than those obtained with [CB09, KROU] and [M05, KROU] with an average offset of 0.15 dex and 0.39 dex, respectively. The difference with respect to M05 is consistent with previous results (e.g., Maraston et al. 2006; van der Wel et al. 2006; Bruzual 2007) in spite of the slight dependence on the mass. However, the 0.16 dex offset between [CB09, KROU] and [M05, KROU] (illustrated for completeness in bottom-right panel of Figure 16) is larger than expected revealing a more complex relative difference between the two libraries beyond the treatment of the TP-AGB phase.

Finally, the bottom-right panel of Figure 15 shows the comparison of the stellar masses estimates obtained with a CAL01 and a Charlot \& Fall (2000, CF00) dust extinction laws for the P01 models and a KROU IMF. The most relevant differences between CAL01 and CF00 extinction laws are that the latter presents a larger attenuation of the stellar component, which effectively leads to lower fluxes (mostly in the UV) for similar values of the extinction. Furthermore, the wavelength dependence of the attenuation in CF00 is grayer (i.e., shallower) than in CAL01. The overall result of the comparison is a small offset of -0.03 dex with a $\sim 0.2$ dex rms, similar to what we found in PG08. This is also in good agreement with the results of Muzzin et al. (2009), indicating that the treatment of the extinction law does not play a major role in the estimate of the stellar mass (although it is more relevant for other estimated parameters).

In summary, we find that after accounting for the different systematic offsets, all models seem to be roughly consistent within a factor two $(\sim 0.3 \mathrm{dex})$. However, there are mass dependent systematics that should be taken into account in the analysis of overall properties of galaxy samples (e.g., Marchesini et al. 2009; Taylor et al. 2009; Ilbert et al. 2010).

\subsubsection{Comparison to other Stellar Mass Catalogs}

In this section, we compare our stellar masses with the estimates from Bundy et al. (2006) and Trujillo et al. (2007). In the former, the authors derived stellar masses for a large sample of galaxies with spectroscopic redshifts from the DEEP2 survey in the EGS. In the latter, the authors combined spectroscopic and photometric redshifts to study the properties of a mass limited sample $\left(\log M>11 \mathcal{M}_{\odot}\right)$. Both works used the same photometric data set consisting of five bands: $B R I$ from the CFHT survey, and $J K$ from the Palomar NIR survey. The stellar masses in both cases were essentially computed based 

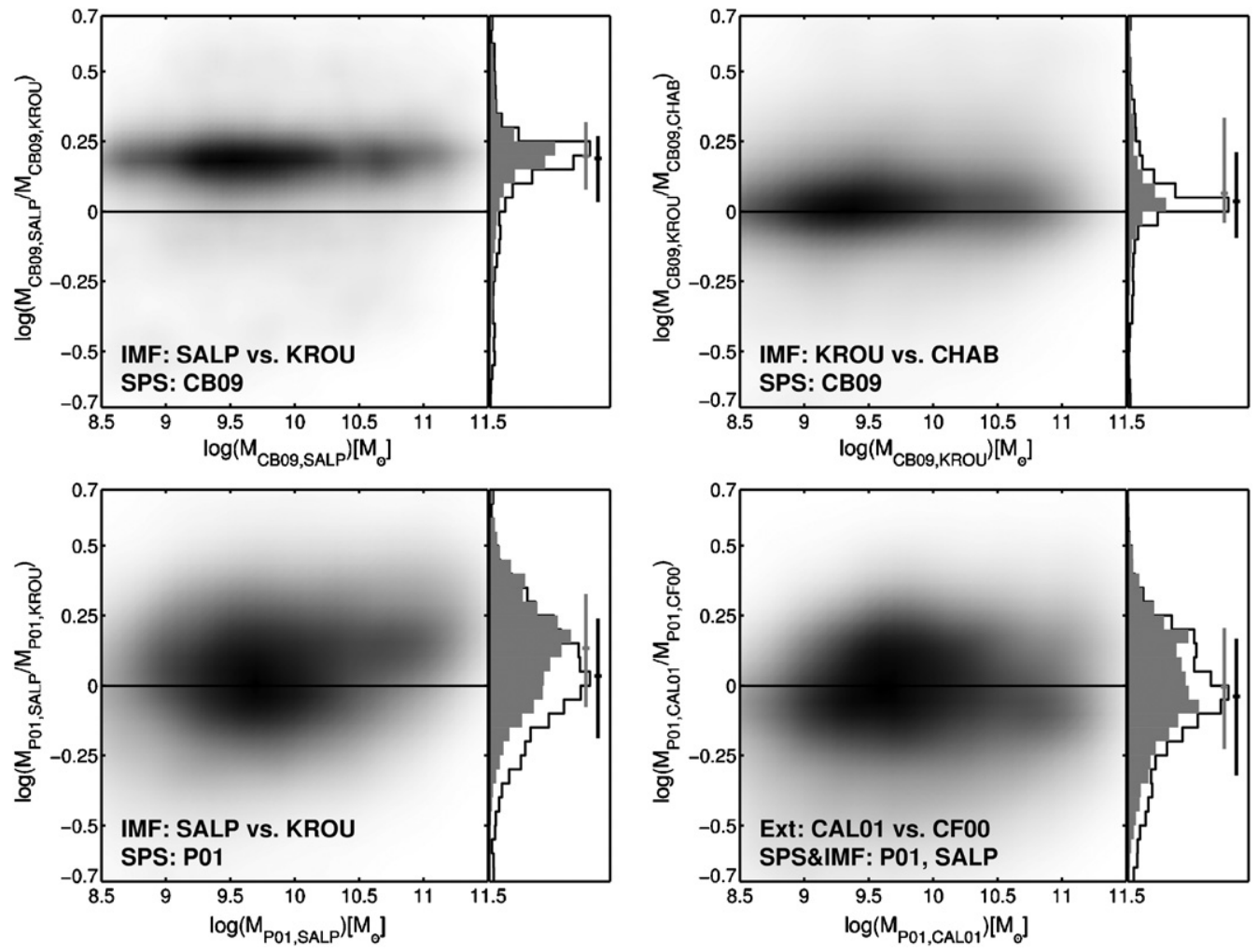

Figure 15. Comparison of the stellar masses obtained using different IMFs and dust extinction laws for a given SPS library. Top left: P01 models with a SALP and KROU IMFs. Top right: CB09 models with a SALP and KROU IMFs. Bottom: CB09 models with a KROU and CHAB IMFs. Bottom right: P01 models with a CAL01 and CF00 extinction laws. The histograms in the right part of the plot depict the ratio of the stellar masses obtained with each IMF for galaxies with $M_{\text {model }}<$ $10 \mathcal{M}_{\odot}$ (empty) and $M_{\text {model }}>10 \mathcal{M}_{\odot}$ (filled). The solid lines above the histogram show the median value and $1 \sigma$ of the distribution at both sides of the median.
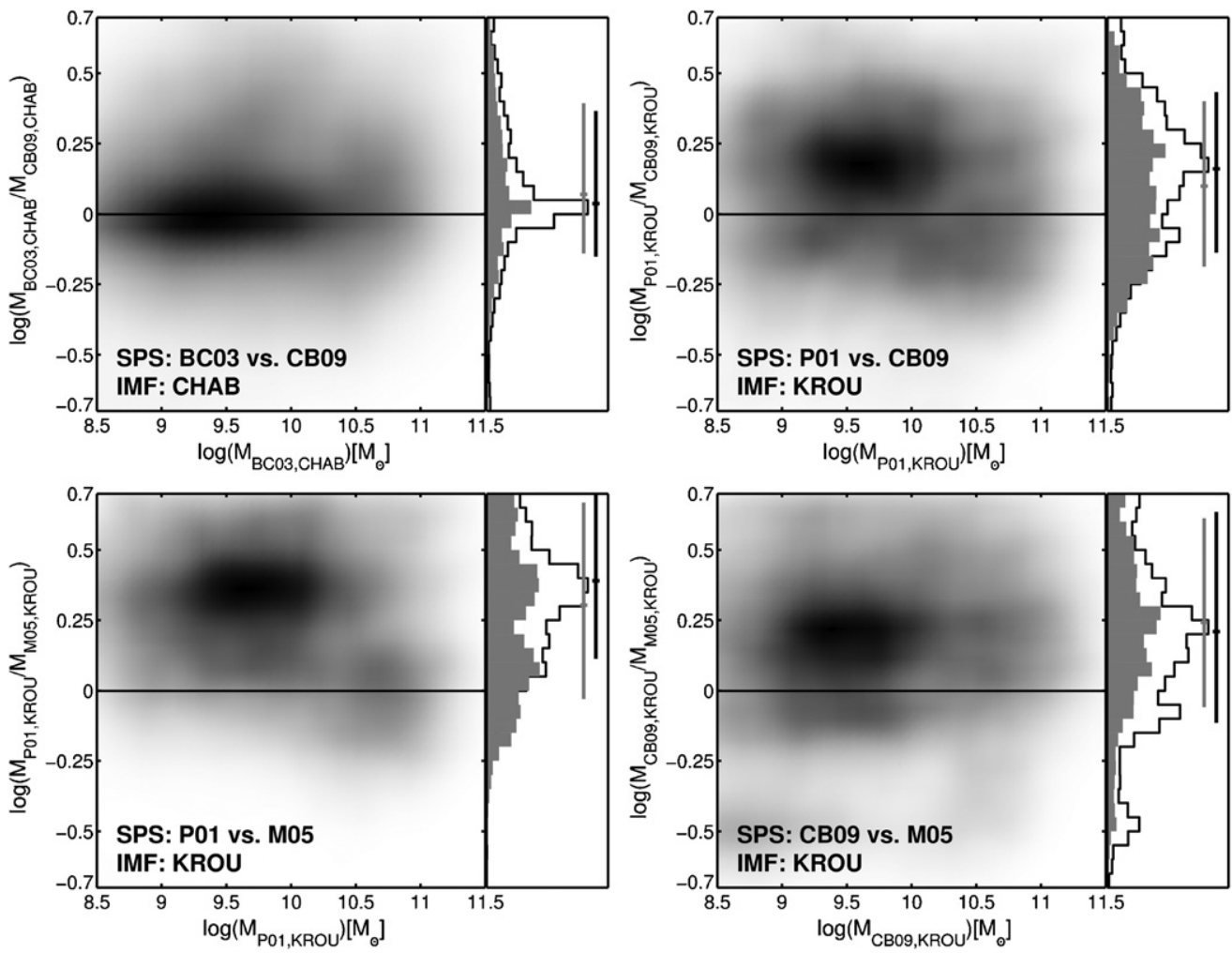

Figure 16. Comparison of the stellar masses obtained using different SPS models with the same IMF. Top left: BC03 and CB09 models with a CHAB IMF. Top right: P01 and CB09 models with a KROU IMF. Bottom left: P01 and M05 models with a KROU IMF. Bottom right: CB09 and M05 models with a KROU IMF. The histograms in the right part of the plot depict the ratio of the stellar masses obtained with each model for galaxies with $M_{\text {model }}<10 \mathcal{M}_{\odot}$ (empty) and $M_{\text {model }}>$ $10 \mathcal{M}_{\odot}$ (filled). The solid lines above the histogram show the median value and $1 \sigma$ of the distribution at both sides of the median. 

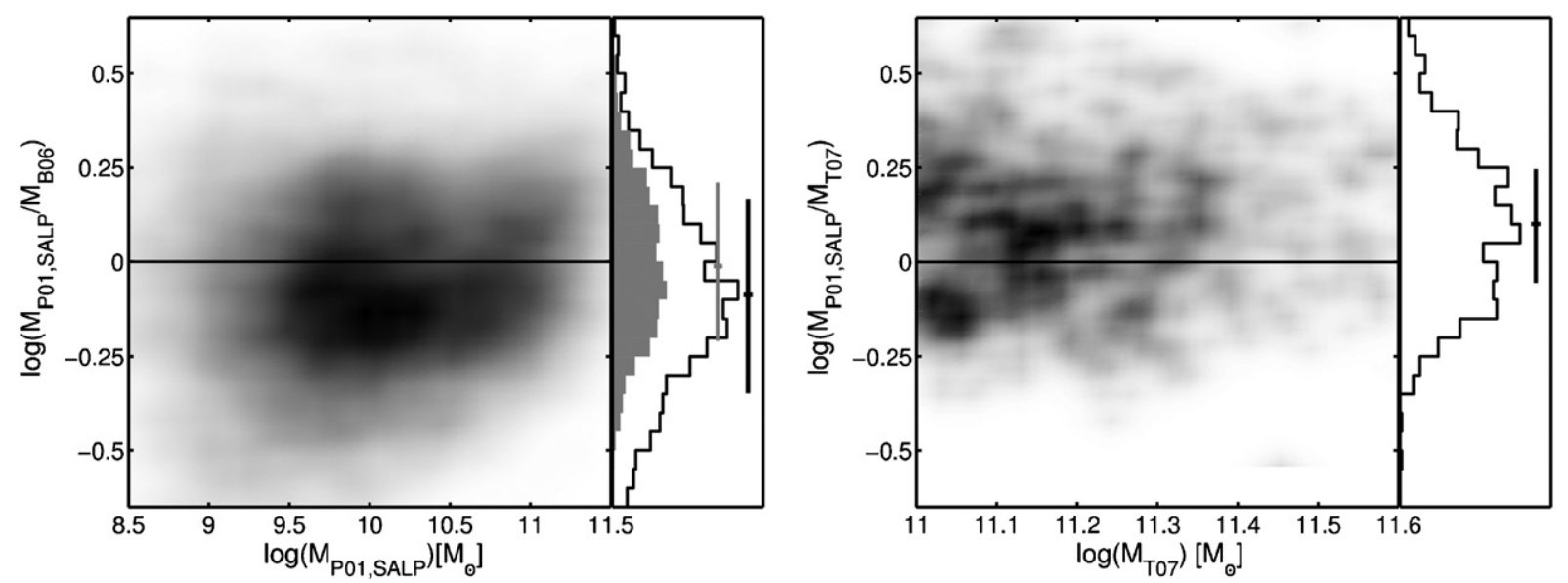

Figure 17. Difference between our best-fit stellar mass using [P01, SALP] and the stellar masses of Bundy et al. (2006) (left), and the mass limited sample (log $M>$ $11 \mathcal{M}_{\odot}$ ) of Trujillo et al. (2007). The histograms in the right side are the same as in Figure 15. Note that the comparison to Trujillo et al. is limited to log $M>11 \mathcal{M}_{\odot}$.

on the fitting of the SEDs to a grid of templates derived from BC03 models with a Chabrier (2003) IMF and exponentially decreasing SFHs. In particular, Bundy et al. used the rest-frame $K$-band luminosity and mass-to-light ratio to scale the templates and compute the probability distribution of the stellar mass and the most likely value. On the contrary, Trujillo et al. (based on the results of Conselice et al. 2007) did not renormalize the templates in a single band but used the whole SED to scale the fluxes, similarly to our approach but restricted to only five bands.

We cross-correlate the catalogs using a $2^{\prime \prime}$ radius, and we double check the validity of the match ensuring that the spectroscopic redshifts (independently matched) are the same. The final sample contains 4706 and 791 galaxies detected in the catalogs of Bundy et al. and Trujillo et al., respectively. For the comparison to Bundy et al. the photometric redshifts were forced to the spectroscopic value and for the comparison to Trujillo et al. the photometric redshifts were forced to the values quoted in their paper.

The left panel of Figure 17 shows the comparison of the stellar masses with Bundy at al. for our default modeling assumptions. Our estimates are slightly lower with a median difference of $\Delta \log (M)=-0.07 \pm 0.21$ dex. Also, we find that the stellar masses computed with [P01,SALP] are in better agreement with Bundy et al. than those obtained using the same modeling configuration as in their work, [BC03, CHAB], which would increase the difference in smaller masses to $\Delta \log (M)=$ -0.12 dex. We further investigate if this offset is caused by a difference in the photometry by comparing our $K$-band magnitudes to those of Bundy et al. that were computed using $2^{\prime \prime}$ radius apertures (for the SED fitting). The sources in Bundy et al. are on average $\Delta K=0.12$ mag fainter than in our catalog, which would imply a larger difference in the stellar masses if we simply scale their magnitudes to our photometry. Thus, the most plausible explanation for this small offset is the use of different techniques for estimating the stellar masses, and specifically the use of IRAC data in our study. The right panel of Figure 17 shows the comparison of the stellar masses with Trujillo at al. The overall comparison presents a good agreement with a median difference of $\Delta \log (M)=0.10 \pm$ 0.25 dex, slightly larger than the offset to masses of Bundy et al. However, the scatter of the distribution is quite similar to that of the comparison to Bundy et al. for the highest stellar masses $\log (M)>11 \mathcal{M}_{\odot}$.

\section{SED ANALYSIS: STAR FORMATION RATES}

In this section, we present the estimations of the SFRs of the galaxies in our IRAC sample based on their UV-to-FIR SEDs. We also discuss the quality of these estimates as well as their associated systematic uncertainties.

The SFR of a galaxy is frequently computed from the UV and IR luminosities through theoretical or empirical calibrations. As young stellar populations emit predominantly in the UV, this wavelength range is highly sensitive to recent events of star formation. However, this UV emission is usually attenuated by dust, which re-emits the absorbed energy in the thermal IR. Consequently, the ongoing SFR can be estimated either by correcting the UV luminosity for extinction or combining the IR emission and the unobscured UV flux.

Here, we focus on the latter approach making use of the high quality FIR fluxes observed with MIPS at 24 and $70 \mu \mathrm{m}$. Thus, assuming that the total SFR of a galaxy can be estimated by summing up two components (see, e.g., Bell et al. 2005; Iglesias-Páramo et al. 2007): the part of the star formation that is probed by a tracer affected by dust attenuation, so we only are able to observe directly a fraction of it (i.e., the unobscured component), and the part of the star formation that is hidden by dust (obscured component). The unobscured star formation can be measured with the rest-frame UV emission, which can be estimated from the optical/NIR SEDs for the galaxies in our sample. The obscured component can be estimated from the total IR thermal emission (thus, we will refer to it as IR-SFR or IR-based SFR). However, its calculation is usually affected by the choice of template libraries fitting the IR part of the SED and, more significantly, by the photometric coverage in the MIR-to-mm spectral range. In this section, we will focus on the analysis of the IR-based SFR and the random and systematic uncertainties associated with the different procedures used to estimate it.

The structure of this section is as follows. First, we describe how we fit the IR part of the SEDs to dust emission models and present the different methods used to estimate an IR-based SFR from monochromatic and integrated luminosities in the MIRto-mm range. Then, we compare these different methods and discuss the systematic and random uncertainties inherent to the calculation of IR-based SFRs.

In this section, the reader must have in mind that the most useful information to estimate IR-based SFRs comes from the 
MIPS $24 \mu \mathrm{m}$ fluxes. The reason is simple: these observations are the deepest in the MIR-to-mm range, so we only have this SFR tracer for the vast majority of sources in our sample. Ideally, it would be desirable to have other fluxes in the IR to constrain the fits to dust emission models, but this is only possible for a very small fraction of galaxies which have MIPS $70 \mu \mathrm{m}$ data, or other photometric points in the (sub)mm. Even with Herschel data, there will be a significant population of galaxies that will only count with the MIPS $24 \mu \mathrm{m}$ flux. Therefore, an important part of our discussion will be assessing the reliability of IR-based SFRs based only on MIPS $24 \mu \mathrm{m}$ data. For that purpose, we will take advantage of the very deep observations carried out at $70 \mu \mathrm{m}$ within the Spitzer FIDEL Legacy Project, studying the variations in the estimated IR-based SFRs fitting MIPS $24 \mu \mathrm{m}$ and MIPS $70 \mu \mathrm{m}$ simultaneously.

\subsection{IR SED Fitting}

Typically, IR-based SFR are computed either from the total IR luminosity, i.e., the integrated emission from 8 to $1000 \mu \mathrm{m}$ [L(TIR)] or from monochromatic luminosities at different wavelengths. Both methods require a detailed characterization of the IR SED, which is usually obtained by fitting the observed fluxes to dust emission templates. However, as mentioned above, these estimates are largely dependent on the choice of templates. An issue that is usually aggravated by the fact that typically the only measurement of the MIR emission comes from the $24 \mu \mathrm{m}$ data, and occasionally $70 \mu \mathrm{m}$, whereas the total IR luminosity is commonly dominated by the emission at longer wavelengths $\lambda \sim 100 \mu \mathrm{m}$.

Thus, in order to study in detail the intrinsic uncertainties in the IR-based SFRs arising from these issues, we follow two different approaches to fit the IR data to the dust templates: (1) we study the galaxies detected at MIPS $24 \mu \mathrm{m}$ fitting only this flux to models of Chary \& Elbaz (2001, CE01 hereafter), Dale \& Helou (2002, DH02 hereafter), which is a usual scenario in studies of the IR-emission at high-z (see e.g., Santini et al. 2009; Wuyts et al. 2008). In this case, we asses the differences between IR-based SFR (hereafter $\operatorname{SFR}_{i}(24)$ ) estimated with several methods, and the impact of using different models; (2) we restrict the analysis to galaxies simultaneously detected in IRAC and MIPS 24 and $70 \mu \mathrm{m}$, fitting all fluxes at rest-frame wavelengths $\lambda>5 \mu \mathrm{m}$ (where the luminosity of a galaxy must present a significant non-stellar contribution; see, e.g., PérezGonzález et al. 2006; Smith et al. 2007) to the models of CE01, DH02, and also Rieke et al. (2009, R09 hereafter). We refer to these galaxies as the best-effort sample and their SFRs (8, $24,70)$. The notation indicates that the fit essentially includes 8,24 , and $70 \mu \mathrm{m}$ data up to $z \sim 0.6,24$, and $70 \mu \mathrm{m}$ data at higher redshift. Based on this sample we can study the impact of having a better constrained IR SED against the MIPS $24 \mu \mathrm{m}$ only scenario (e.g., as in Kartaltepe et al. 2010). In both cases, the fitting is carried out by fixing the redshift to $z_{\text {phot }}$ or $z_{\text {spec }}$ (if available). Then, the excess resulting from subtracting the predicted contribution from the stellar flux (given by the bestfitting optical template) to the MIR bands is fitted to each set of models. In the case when only MIPS $24 \mu \mathrm{m}$ data are used, the templates are not fitted but rather scaled, i.e., we obtain the rest-frame monochromatic luminosity for that flux and redshift and we select the most likely template based in their absolute normalization in the total IR luminosity (as in, e.g., Papovich et al. 2006; Santini et al. 2009). Moreover, for sources undetected in the $24 \mu \mathrm{m}$ data, we set an upper limit of $f(24)=60 \mu \mathrm{Jy}$, the approximate SNR $=5$ level of the MIPS data in EGS (see Paper I for more details), which allows us to provide an upper limit of the IR-based SFR. Figure 2 shows an example of the IR SED fitting jointly with the optical template.

Based on the best-fitting templates, we computed several IR monochromatic and the integrated luminosities $[L(\lambda)$ and $L$ (TIR), respectively] as the median value of all the fitted template sets. In the following, we describe various possibilities for IR-based SFRs based on $L(\lambda)$ at different wavelengths. These relations are calibrated from galaxy samples counting with extensive IR coverage (at least more than 3-4 bands), and provides an alternative estimate of the SFR based on milder template extrapolation than $L(\mathrm{TIR})$, which in principle makes them more robust when only few bands are available for the fitting. Note however that the rest-frame wavelengths around $10-30 \mu \mathrm{m}$ are wildly variable and thus extrapolating luminosities in this region involve significant uncertainties, e.g., $L(8)$ based on $24 \mu \mathrm{m}$ data at $z \sim 2$ (see Section 5.3.2 for more details).

\subsection{Total SFR and IR-based SFRs Estimates}

Our method to estimate the total SFRs is based on a combination of the IR emission and the unobscured UV flux (similarly to Pérez-González et al. 2006; Kennicutt et al. 2009; Santini et al. 2009). In particular, we use the prescription of Bell et al. (2005, see also Papovich et al. 2007), which is based on the calibration for the total IR luminosity of Kennicutt (1998) and parameterizes the contribution of radiation that escapes directly in the UV:

$$
\mathrm{SFR}=\mathrm{SFR}_{\mathrm{TIR}}+\mathrm{SFR}_{\mathrm{UV}, \mathrm{obs}}
$$

$\operatorname{SFR}\left(M_{\odot} \mathrm{yr}^{-1}\right)=1.8 \times 10^{-10}[L(\mathrm{TIR})+3.3 \times L(0.28)] / L_{\odot}$,

where $L(\mathrm{TIR})$ is the integrated total IR luminosity and $L(0.28)$ is the rest-frame monochromatic luminosity at $0.28 \mu \mathrm{m}$ (uncorrected for extinction). The well-sampled SEDs of our galaxies at optical wavelengths allow a robust estimation of $L(0.28)$ by interpolating in the best-fitting optical template. However, as described in the previous section, the value of $L$ (TIR) is strongly model dependent, as it is based on an extrapolation from one or a few MIR fluxes to the total emission from 8 to $1000 \mu \mathrm{m}$. An alternate possibility is to obtain other IR-based SFRs based on $L(\lambda)$, thus reducing the template dependence. In the following, we will refer to the IR-based SFR derived from $L$ (TIR) as $\mathrm{SFR}_{\mathrm{TIR}}$. In addition, we compute four other IR-based estimates.

The first estimate is based on rest-frame monochromatic luminosity at $8 \mu \mathrm{m}$ (hereafter $\mathrm{SFR}_{\mathrm{B} 08}$ ). These estimate make use of the empirical relation between $L(8 \mu \mathrm{m})$ and $L(\mathrm{TIR})$ described in Bavouzet et al. (2008) and the Kennicutt factor to transform to SFR:

$$
\operatorname{SFR}_{\mathrm{B} 08}\left(M_{\odot} \mathrm{yr}^{-1}\right)=1.8 \times 10^{-10} \times\left(377.9 \times L(8)^{0.83}\right) / L_{\odot} .
$$

The second method in based on Equation (14) of Rieke et al. (2009) that relates the SFR (hereafter $S_{F R}$ R9) to the observed flux in the MIPS $24 \mu \mathrm{m}$ band and the redshift. The redshift dependent coefficients of the relation were computed using averaged templates derived from a set of empirical IR-SEDs fitting local galaxies. This estimation of the SFR is independent of the rest, as it is based on different templates. The conversion from IR luminosities to SFRs is also computed through the Kennicutt factor. However, the authors scaled the factor to a Kroupa-like (2001) IMF (the original factor is for a SALP IMF) 
multiplying it by 0.66 (a similar conversion is obtained in Salim et al. 2007). Here, we undo that change for consistency with the other methods that are computed using the factor for a SALP IMF.

The last method is not strictly an IR-based SFRs but an estimate the global SFR. It is based on the empirical relation given in Alonso-Herrero et al. (2006) between the rest-frame monochromatic luminosity at $24 \mu \mathrm{m}$ and the SFR (hereafter, $\mathrm{SFR}_{\mathrm{A}-\mathrm{H} 06}$ ),

$$
\operatorname{SFR}_{\mathrm{A}-\mathrm{H} 06}\left(M_{\odot} \mathrm{yr}^{-1}\right)=1.51 \times 10^{-8} \times L(24)^{0.871} / L_{\odot} .
$$

This formula is based on the calibration of $L(\operatorname{Pa} \alpha)$ versus $L(24 \mu \mathrm{m})$ obtained for a set of local ULIRGS using the Kennicutt (1998) relation between $L(\mathrm{~Pa} \alpha)$ and SFR. A similar result was obtained by Calzetti et al. (2007) for resolved star-forming regions in local starburst (see also Kennicutt et al. 2009). This estimation refers to the global SFR, not the IRbased SFR, because the empirical relation in Alonso-Herrero et al. (2006) already takes into account the unobscured star formation (measured through the observed $\mathrm{Pa} \alpha$ emission) and the extinction correction (applied to calculate $L(\mathrm{~Pa} \alpha)$ in that paper).

\subsection{Accuracy of the IR-based SFRs}

In the following sections, we analyze the systematic uncertainties in the IR-based SFRs associated with the use of different models and indicators, and also the number of photometric bands available for IR SED fitting.

First, we compare the values obtained with each of the methods presented in the previous section for a sub-sample of MIPS $24 \mu \mathrm{m}$ detected galaxies. In this case, the SED is fitted to $24 \mu \mathrm{m}$ data only (Section 5.3.1). Note that we have chosen several methods for estimating the IR-based SFR that present intrinsically different approaches, using either integrated and monochromatic luminosities or observed fluxes. Here, we also test the differences introduced by the use of the CE01, DH02, or R09 models. For simplicity, in this case, the comparison to the R09 models is done through the SFRs obtained with their empirical relation $\left(\mathrm{SFR}_{\mathrm{R} 09}\right)$ instead of fitting the data to the three models.

Second, we study the differences in the SFRs obtained for MIPS $24 \mu \mathrm{m}$ sample and the best-effort sample, which count with a better IR coverage based on IRAC-8.0 plus MIPS 24 and $70 \mu \mathrm{m}$ data (Section 5.3.2). With this test we quantify the systematic effects associated with use of limited IR data. Finally, we repeat the comparison of values obtained with each method for the best-effort sample including also highly accurate SFRs drawn from other authors based on a more detailed IR coverage (Section 5.3.3). Based on this comparison we asses the goodness of our best-effort SFRs and the reliability of the different methods studied here.

For the sake of clarity, we will refer all the comparisons between the SFRs estimated with each method to SFR TIR $_{\text {which, }}$ as explained in Section 5.1, is computed from the average total infrared luminosity of all the fitted template sets. In addition, we will refer to them just as SFRs (dropping the IR prefix). In the case of $\mathrm{SFR}_{\mathrm{A}-\mathrm{H} 06}$, the proper IR-based SFRs are obtained by subtracting the contribution of the SFR $\mathrm{UV}_{\text {, obs. Nevertheless, as }}$ our working samples are composed by strong IR-emitters, we are biased toward dust obscured galaxies where this contribution is presumably small. For example, Figure 18, which shows the ratio $\mathrm{SFR}_{\mathrm{UV} \text {,obs }} / \mathrm{SFR}_{\mathrm{TIR}}$, indicates that $\mathrm{SFR}_{\mathrm{UV} \text {,obs }}$ is lower than $\mathrm{SFR}_{\mathrm{TIR}}$ (in most cases), with a clear trend for galaxies

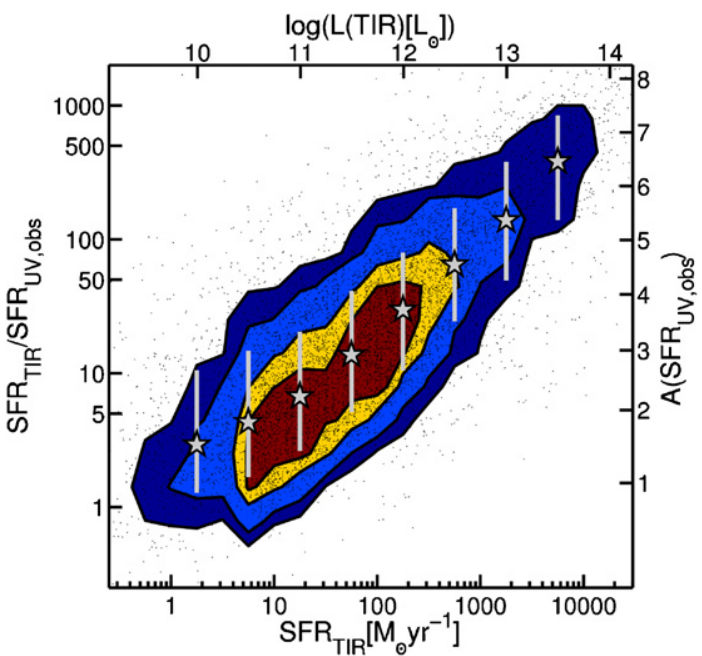

Figure 18. Ratio of the two components of the total SFR (SFR $=\mathrm{SFR}_{\mathrm{UV} \text {,obs }}+$ $\mathrm{SFR}_{\mathrm{IR}}$; Equation (4)) as a function of the $\mathrm{SFR}_{\mathrm{TIR}}$ for a sub-sample galaxies detected in MIPS $24 \mu \mathrm{m}(f(24)>60 \mu \mathrm{Jy})$. Each contour contains (from the inside out) $25 \%, 50 \%, 75 \%$, and $90 \%$ of the sample, respectively. The underlying black dots depict the individual values of the ratio of SFRs. The gray stars with error bars depict the median and $1 \sigma$ of the ratio of SFRs in bins of SFRTIR.

with intense star formation to present more and more extincted starbursts.

\subsubsection{Analysis of IR-based SFRs: MIPS $24 \mu \mathrm{m}$ Sample}

Figure 19 shows the comparison of the IR-based SFRs obtained with the different methods presented in Section 5.2 with respect to $\mathrm{SFR}_{\mathrm{TIR}}$ as a function of SFR and redshift. All the estimates discussed in this section are based on $24 \mu \mathrm{m}$ data only, i.e., $\operatorname{SFR}_{i}(24)$. We omit the parentheses for simplicity. The $\mathrm{SFR}_{\mathrm{TIR}}$ estimated separately with the models of CE01 (green dots) and DH02 (blue dots) are shown jointly with the median and rms of both values in several SFR and luminosity bins. The typical scatter of the SFRs estimated with both libraries is smaller than $\sim 0.3$ dex, consistent with the results by other authors (Marcillac et al. 2006; Papovich et al. 2007). When we compare the CE01 and DH02 libraries as a function of redshift, the maximum differences are observed for galaxies at $z=1-2$. For these sources, the estimates with the DH02 models are larger than those with CE01 models, as found by Santini et al. (2009). In this redshift range, the $24 \mu \mathrm{m}$ band is probing the spectral region where the $9.6 \mu \mathrm{m}$ silicate absorption is found, jointly with the prominent PAHs around 7-9 $\mu \mathrm{m}$. The shape of the models in CE01 and DH02 template sets is very different in this region, with the former presenting less prominent PAH features than the latter. In fact, all the DH02 models are identical below $\sim 9 \mu \mathrm{m}$, while CE01 models present a wide variety of spectral shapes, with a rising warm-dust continuum hiding the PAH features between 6 and $20 \mu \mathrm{m}$ as we move to models with higher IR luminosities.

The values of $\mathrm{SFR}_{\mathrm{A}-\mathrm{H} 06}$ are systematically smaller than $\mathrm{SFR}_{\mathrm{TIR}}$. For $\mathrm{SFR}_{\mathrm{TIR}}>100 M_{\odot} \mathrm{yr}^{-1}$, we find $\Delta \mathrm{SFR}=-0.18 \pm$ 0.05 dex. For smaller values of the SFR, where the unobscured and obscured star formation are comparable, $\mathrm{SFR}_{\mathrm{A}-\mathrm{H} 06}$ is down to a factor of 0.6 dex smaller than $S_{\text {SFR }}$, with a larger scatter.

The comparison of $\mathrm{SFR}_{\mathrm{TIR}}$ and $\mathrm{SFR}_{\mathrm{B} 08}$ clearly indicates that the empirical relations $L[8]$-to- $L_{\mathrm{TIR}}$ in Bavouzet et al. (2008) (Equation (5)) and in the models of CE01 and DH02 (see, e.g., Figure 8 of Daddi et al. 2007) are substantially different. The ratio of the two SFRs as a function of SFR $\mathrm{TIR}_{\text {in }}$ is tiled 

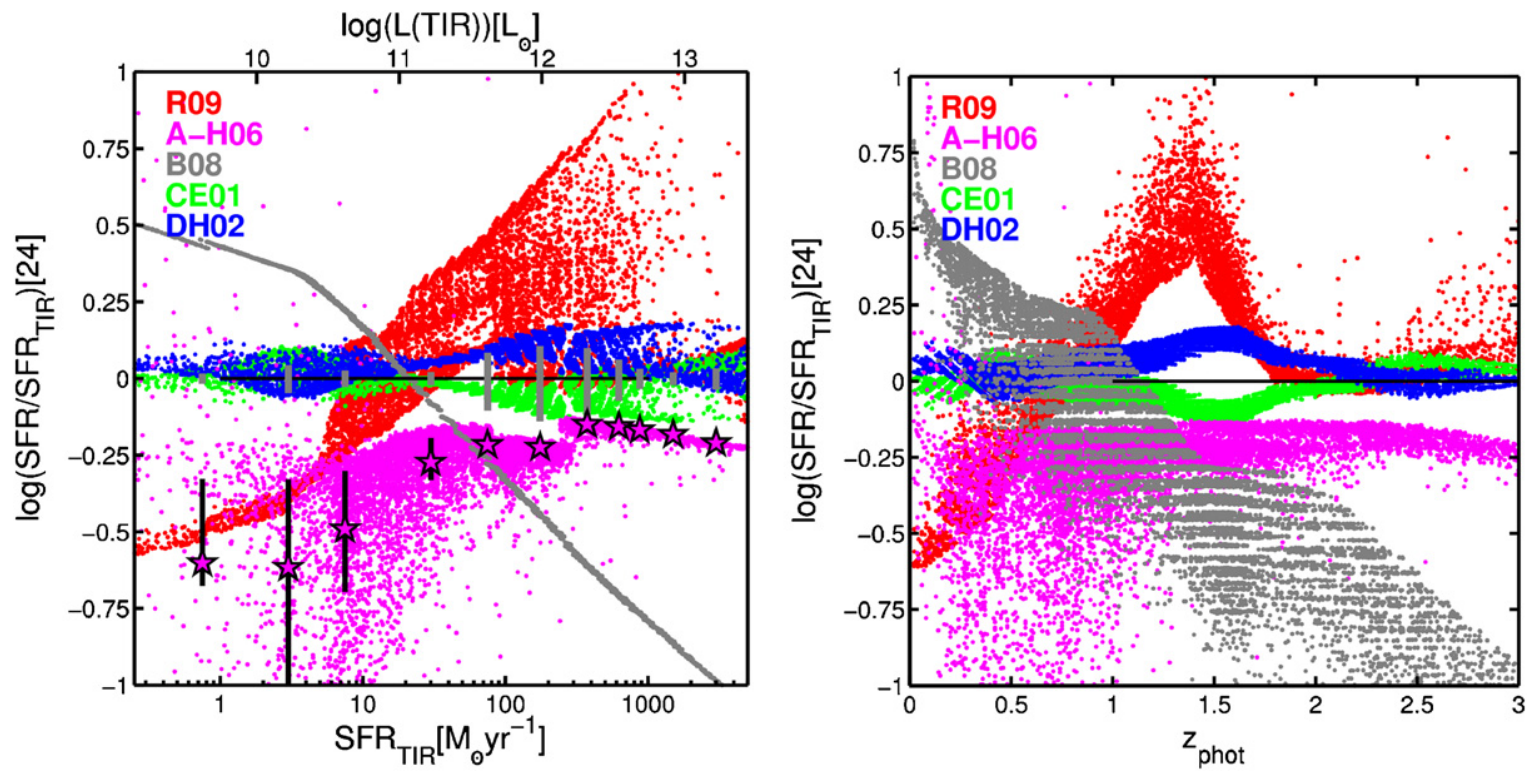

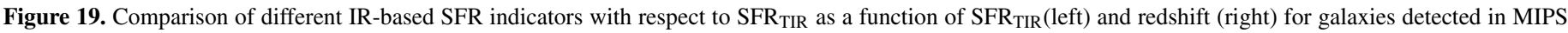

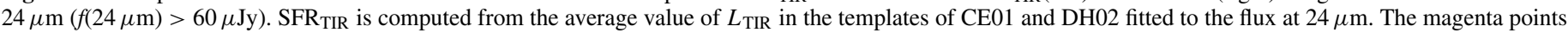

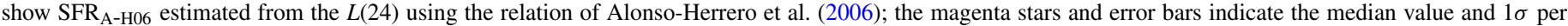

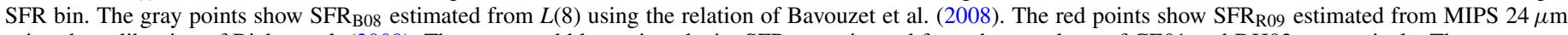

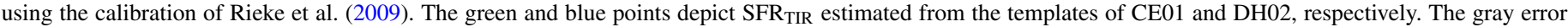
bars depict the $1 \sigma$ uncertainty in $\mathrm{SFR}_{\mathrm{TIR}}$ per SFR bin.

with respect to the unity line, and consequently, both estimates are only consistent within a narrow interval around SFR $20 M_{\odot} \mathrm{yr}^{-1}$ (or $z \sim 1$ ). For SFR $\mathrm{TIR}>100$ and $1000 M_{\odot} \mathrm{yr}^{-1}$ (the latter being the typical value for the $z \sim 2$ galaxies detected by MIPS) the $\mathrm{SFR}_{\mathrm{B} 08}$ values are 0.3 and 0.8 dex lower than the SFR $\mathrm{TIR}_{\mathrm{T}}$ estimates, respectively. In contrast, for $\mathrm{SFR}_{\mathrm{TIR}} \lesssim$ $10 M_{\odot} \mathrm{yr}^{-1}, \mathrm{SFR}_{\mathrm{B} 08}$ is larger than $\mathrm{SFR}_{\mathrm{TIR}}$ by $>0.2$ dex.

The equation to calculate $\mathrm{SFR}_{\mathrm{R} 09}$ (Rieke et al. 2009) varies with redshift. Consequently, the $\mathrm{SFR}_{\mathrm{R} 09} / \mathrm{SFR}_{\mathrm{TIR}}$ ratio presents different trends as a function of both luminosity and redshift. In terms of redshift, we distinguish three regions: $0<z<$ $1.4,1.4<z \lesssim 1.75$, and $z \gtrsim 1.75$. At $z=0-1.4$, the ratio increases with redshift from an average value of -0.5 dex at $z=0$ to 0.5 dex at $z \sim 1.4$, being close to unity at $z \sim 0.75$. In the interval from $1.4<z \lesssim 1.75$, the ratio decreases from 0.5 dex to nearly $\sim 0$. Finally, at $z>1.75, \mathrm{SFR}_{\mathrm{R} 09}$ values become roughly consistent with $\mathrm{SFR}_{\mathrm{TIR}}$ with little scatter up to $z=3$, $\Delta \mathrm{SFR}=0.02_{0.04}^{0.06}$ dex. These large differences are related to the distinct shapes of the R09 and CE01/DH02 templates. At $z \lesssim 0.5$, the $24 \mu \mathrm{m}$ band probes a spectral range dominated by warm dust and emission features found by Spitzer at $\lambda \sim 17 \mu \mathrm{m}$ and identified with PAH or nanoparticles (Werner et al. 2004). At these redshifts, our sample is dominated by galaxies with $L(\mathrm{TIR}) \sim 10^{10} L_{\odot}$, and the CE01 models for these luminosities differ from the corresponding R09 templates by up to 0.5 dex in the $\lambda=16-20 \mu \mathrm{m}$. This explains the differences at low redshift in the right panel of Figure 19. At $z=0.5-1.0$, our sample is dominated by LIRGs, and CE01 and R09 models for $L$ (TIR) $10^{10.75} L_{\odot}$ and $L\left(\right.$ TIR) $\sim 10^{11.25} L_{\odot}$ are very similar (up to $\lambda=1.5 \mathrm{~mm}$ ), resulting on very similar estimates of the SFR. At $z \sim 1.4$, the $24 \mu \mathrm{m}$ band probes the spectral region around $10 \mu \mathrm{m}$, and the galaxies detected by MIPS in this range have $L(\mathrm{TIR}) \gtrsim 10^{11.5} L_{\odot}$. For this luminosity, the CE01 and R09 models differ considerably due to the relative strength of the silicate absorption. For example, for an $L($ TIR $) \gtrsim 10^{12.25} L_{\odot}$, the R09 template predicts a luminosity at $10 \mu \mathrm{m}$ which is a factor of $\sim 0.7$ dex smaller than the CE01 model corresponding to the same $L$ (TIR). Below $8 \mu \mathrm{m}$, rest-frame, the CE01 and R09 models are almost identical for LIRGs and ULIRGs, explaining the good match between $\mathrm{SFR}_{\mathrm{TIR}}$ and $\mathrm{SFR}_{\mathrm{R} 09}$ at $z \gtrsim 2$.

In summary, we conclude that whereas $\mathrm{SFR}_{\mathrm{A}-\mathrm{H} 06}$ and $\mathrm{SFR}_{\mathrm{TIR}}$ are roughly consistent within $\sim 0.3$ dex (modulo a constant offset), regardless of the models used to fit the IR-SED, the values of $\mathrm{SFR}_{\mathrm{B} 08}$ and $\mathrm{SFR}_{\mathrm{R} 09}$ present systematic deviations with respect to those that are not consistent within the typical rms. Moreover, these differences are not constant, but present a dependence of both redshift and SFR. As a result, large systematic offsets (of \pm 0.5 dex) with respect to $\mathrm{SFR}_{\mathrm{TIR}}$ are expected at certain redshifts, e.g., $\Delta \mathrm{SFR} \sim+0.5$ and $-0.5 \mathrm{dex}$ for $\mathrm{SFR}_{\mathrm{R} 09}$ and $\mathrm{SFR}_{\mathrm{B} 08}$ at $z \sim 1.4$, respectively.

\subsubsection{Analysis of IR-based SFRs: Best-effort versus MIPS $24 \mu \mathrm{m}$}

Here, we study the impact on the IR-based SFRs of modeling the IR-SED with limited photometric data. For that matter, we quantify the differences in the SFRs estimated with each of methods compared in the previous section using the sample characterized with MIPS $24 \mu \mathrm{m}$ data and with 8,24 , and $70 \mu \mathrm{m}$ data, i.e., the best-effort sample (note that $\lesssim 2 \%$ of the sample in detected in MIPS $70 \mu \mathrm{m}$ for $\sim 20 \%$ in MIPS $24 \mu \mathrm{m}$ ). In principle, the inclusion of additional mid-IR fluxes must improve the quality of the estimates given that there is a better sampling of the IR SED from which better $k$-corrections to the monochromatic luminosities can be obtained. On the downside, the spectral range probed by the MIPS bands gets narrower with redshift, and the $24 \mu \mathrm{m}$ channel shifts progressively into PAH region, where models are more uncertain and different libraries differ significantly. Also, as the observed $70 \mu \mathrm{m}$ moves further away from the tip of the IR-emission $(\sim 100 \mu \mathrm{m})$, the uncertainty in the extrapolated $L(T I R)$ increases.

Figure 20 shows the ratio $\operatorname{SFR}(24) / \operatorname{SFR}(8,24,70)$ as a function of redshift for each of the different methods to estimate the SFR, except for $\mathrm{SFR}_{\mathrm{R} 09}$, that only depends on the observed 

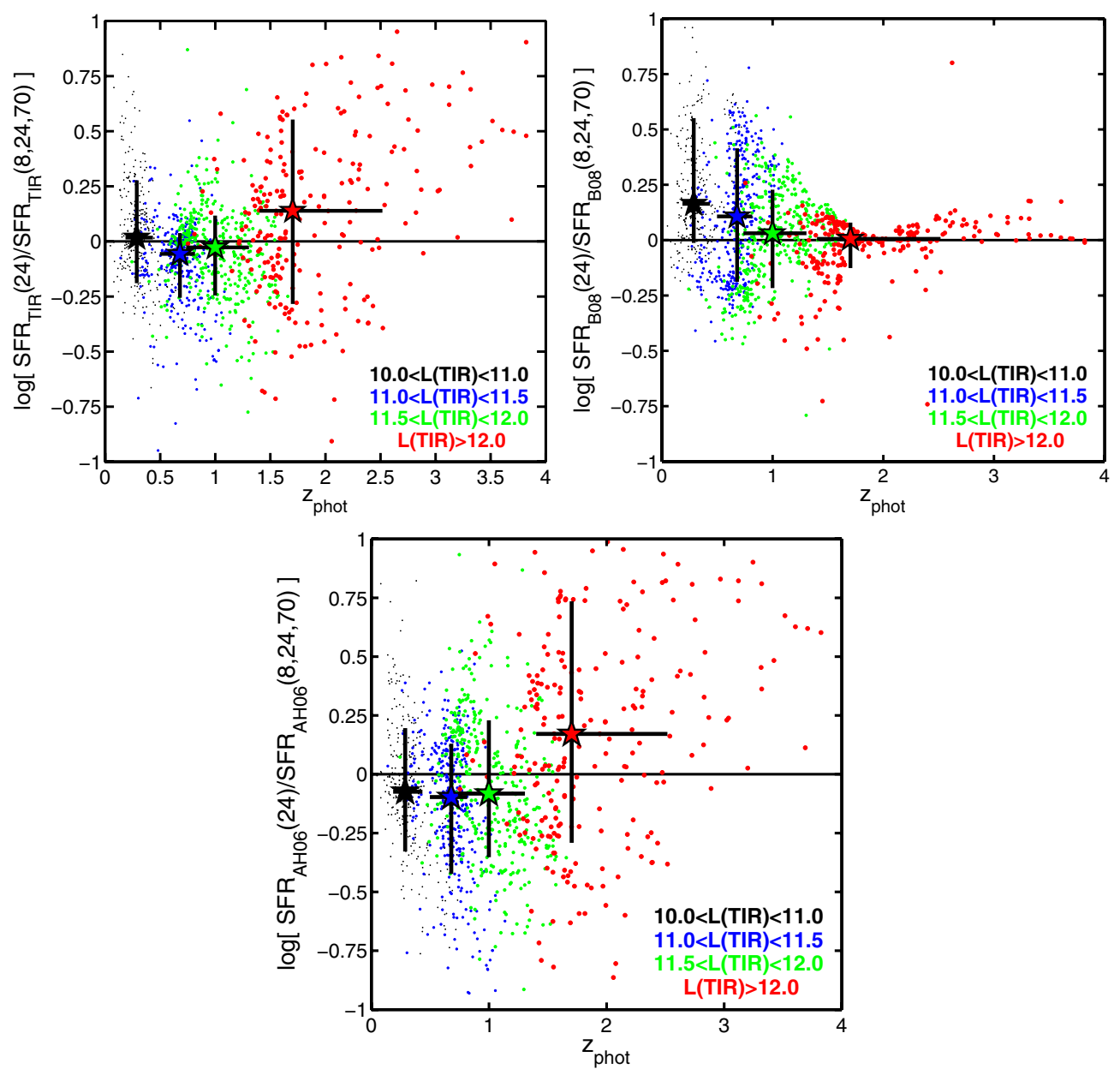

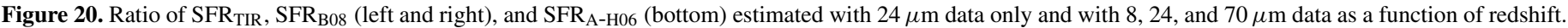

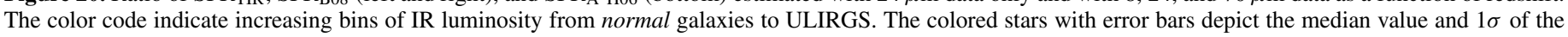
ratio of SFRs and the redshift per luminosity bin.

flux at $24 \mu \mathrm{m}$ and the redshift. The color code indicates four different bins of infrared luminosity. The colored stars with error bars depict the median value and $1 \sigma$ of the redshift and the ratio of SFRs $(24 / 8,24,70)$ for different luminosity bins.

The upper-left panel of Figure 20 shows that $\operatorname{SFR}_{\mathrm{TIR}}(24)$ and $\operatorname{SFR}_{\text {TIR }}(8,24,70)$ are $\sim 1 \sigma$ consistent within $\sim 0.20$ dex up to ULIRG luminosities, showing a small offset (mostly at $z \gtrsim$ 0.5 ) in $\operatorname{SFR}_{\mathrm{TIR}}(24)$ toward underestimating the SFR by $\Delta \mathrm{SFR}=$ $-0.05 \pm 0.20$ dex. On the other hand, ULIRGs (typically at $z \gtrsim$ 1.5) present values of $\operatorname{SFR}_{\mathrm{TIR}}(24)$ larger than $\operatorname{SFR}_{\mathrm{TIR}}(8,24,70)$ with an average difference of $\triangle \mathrm{SFR}=0.15 \pm 0.40 \mathrm{dex}$. This is consistent with the results found for ULIRGs at this redshift by several authors, who report excesses of a factor of 2-10 in the SFRs estimated from MIPS $24 \mu \mathrm{m}$ only (Daddi et al. 2007; Papovich et al. 2007; Rigby et al. 2008). Note that estimating the IR-luminosities for these galaxies based on MIPS $24 \mu \mathrm{m}$ data alone is intrinsically difficult as this band is probing the most variable region of the IR-SED, featuring emission from PAHs and silicate absorptions. In fact, further motive for these discrepancies could associated with a change in the relative strength of these components in high- $z$ galaxies with respect to the local templates, particularly for the ULIRG templates.

Figure 21 presents further evidence of this issue. The left and right panels of the Figure show the rest-frame SED normalized to the flux at $24 \mu \mathrm{m}$ for galaxies at $\tilde{z}=0.7$ and $\tilde{z}=1.5$ in different $L$ (TIR) ranges. The red and black lines are the dust- emission templates of Rieke et al. (2009). The black templates are those corresponding to the IR-luminosity range shown in the legend. Note that we have selected the redshift ranges and template normalization with the specific aim of stressing the differences in warm-to-cold dust colors between the R09 models and the actual observations. The same differences apply to other template sets. The vertical line to the right of the MIPS $70 \mu \mathrm{m}$ data depicts the median and $1 \sigma$ of the distribution of MIPS $70 \mu \mathrm{m}$ fluxes (normalized to $24 \mu \mathrm{m}$, i.e., the $\mathrm{S}_{70} / \mathrm{S}_{24}$ color). At $z \sim 0.7$, the models for an $L(\mathrm{TIR})=10^{10.5}-10^{11.0} L_{\odot}$ nicely predict the actual colors observed for galaxies (the templates plotted in black match the median and $1 \sigma$ range of observed colors). However, at $z \sim 1.5$, galaxies present smaller colors than what the models for the appropriate luminosity range predict. This suggests that the excess in $\operatorname{SFR}_{\mathrm{TIR}}(24)$ could be related to a difference in spectral shapes for ULIRGs at high redshift in comparison with local ULIRGs either due to the strength of the PAH and the silicate features (Elbaz et al. 2010; PérezGonzález et al. 2010) or due to additional continuum emission by an obscured AGN (Daddi et al. 2007).

The lower panel of Figure 20 shows $\operatorname{SFR}(24) / \operatorname{SFR}(8,24,70)$ for the B08 recipe. The ratio of SFRs presents just the opposite trend of what we find for $\mathrm{SFR}_{\mathrm{TIR}}$, i.e., the offset and rms of the comparison are larger at lower redshifts and almost nonexistent $(<0.1 \mathrm{dex})$ at high $-z$. This is not surprising considering that MIPS $24 \mu \mathrm{m}$ shifts toward $8 \mu \mathrm{m}$ with increasing redshifts, 

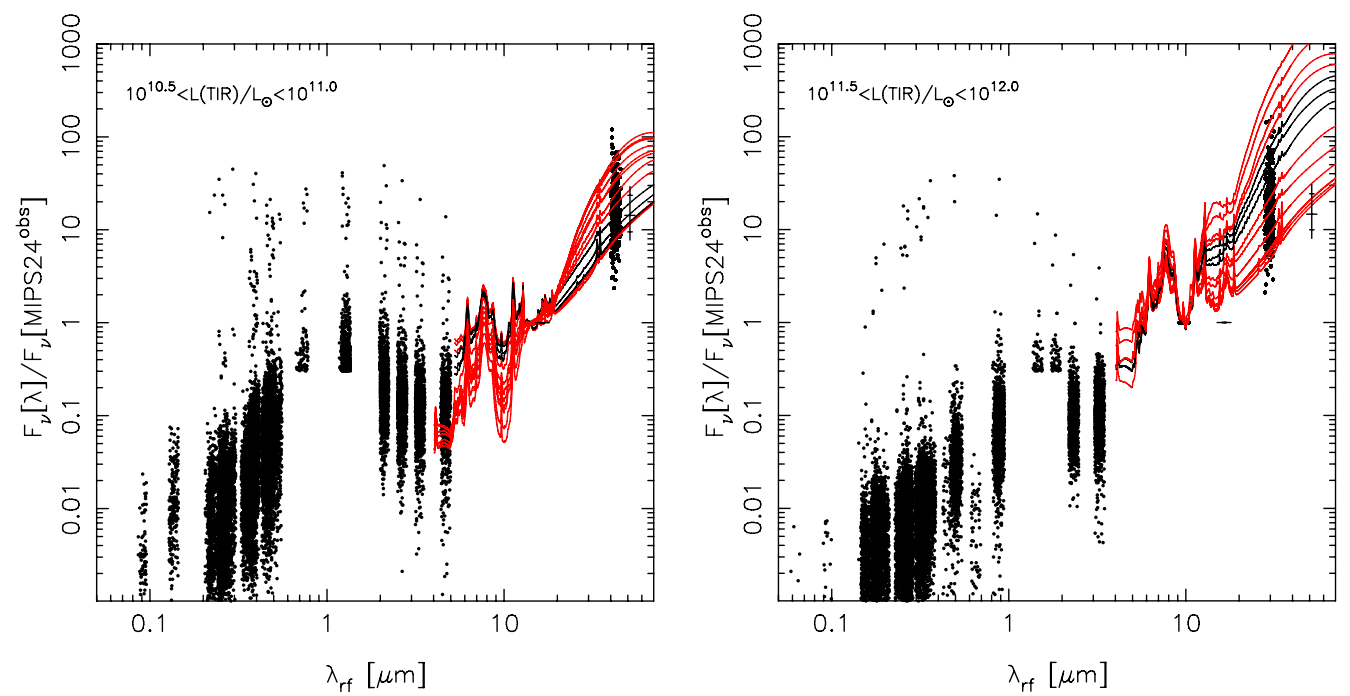

Figure 21. Rest-frame SEDs of galaxies detected in MIPS 24 and $70 \mu \mathrm{m}$ at $0.6<z<0.8$ with $10^{10.5}<L$ (TIR) $<10^{11.0}$ (left panel) and $1.3<z<1.6$ with $10^{11.5}<$ $L(T I R)<10^{12}$ (LIRGS) (right panel). The fluxes are normalized to the flux in MIPS $24 \mu \mathrm{m}$. The solid lines depict the templates of Rieke et al. (2009): black lines are for templates with $L$ (TIR) within the corresponding IR-luminosity range, red lines are for the rest of the templates. The vertical line and marks on the right of the MIPS $70 \mu \mathrm{m}$ fluxes show the median, quartiles, and $1 \sigma$ of the distribution of observed fluxes.
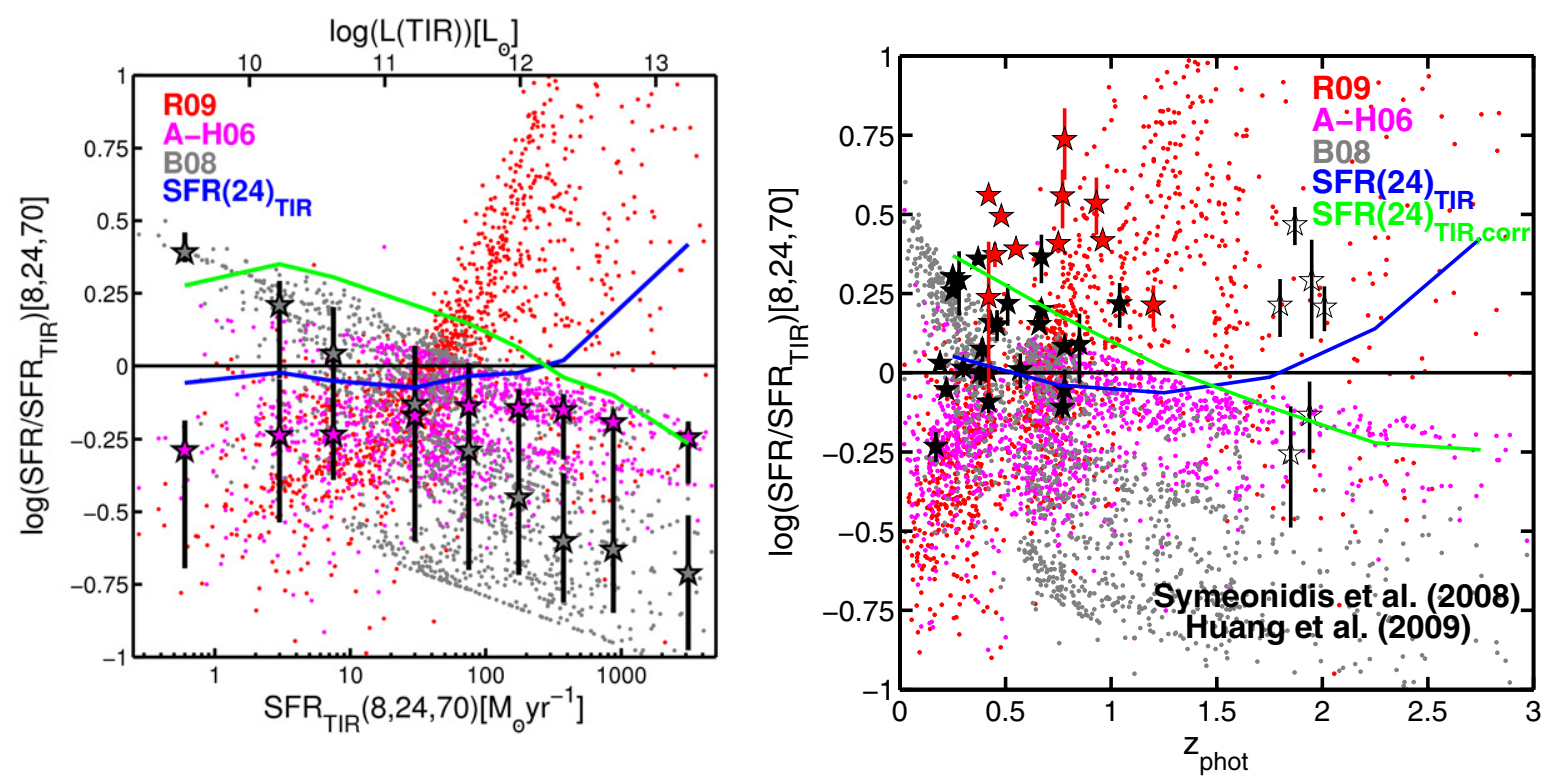

Figure 22. Comparison of different IR-based $\operatorname{SFR}(8,24,70)$ indicators with respect to $\operatorname{SFR}_{\mathrm{TIR}}(8,24,70)$ as a function of $\operatorname{SFR}_{\mathrm{TIR}}$ (left) and redshift (right) for galaxies detected in MIPS $24 \mu \mathrm{m}(f(24 \mu \mathrm{m})>60 \mu \mathrm{Jy})$ and MIPS $70 \mu \mathrm{m}(f(70 \mu \mathrm{m})>3500 \mu \mathrm{Jy})$. The color code is the same as in Figure 19. Here we also show the median values and $1 \sigma$ of the comparison to $\operatorname{SFR}_{\mathrm{B} 08}$ (gray stars with error bars). The blue line joins the median values of $\operatorname{SFR}_{\operatorname{TIR}}(24) / \operatorname{SFR}_{\operatorname{TIR}}(8,24,70)$ per luminosity bin (similarly to the colored stars in the top-left panel of Figure 19). The black stars depict the ratio of SFRs for galaxies in common with the sample of Symeonidis et al. (2008, filled stars at $z<1.2)$ and Huang et al. (2009, open stars at $z \sim 2)$. The IR-based SFR for these sources is one of the most accurate available at the moment, and it is in overall good agreement with our estimates with SFR $\mathrm{TIR}_{\text {. }}$

reducing the impact of the $k$-corrections. As a result, $\mathrm{SFR}_{\mathrm{B} 08}$ is nearly insensitive to the inclusion of $70 \mu \mathrm{m}$ data at $z \sim 2$. Note however that this does not mean that it is a better estimation of the SFR. At $z \lesssim 0.5, \mathrm{SFR}_{\mathrm{B} 08}(24)$ is larger than $\operatorname{SFR}_{\mathrm{B} 08}(8,24$, 70) by $\Delta \mathrm{SFR}=0.18 \pm 0.23 \mathrm{dex}$.

Finally, the right panel of Figure 20 shows the comparison of $\operatorname{SFR}(24) / \operatorname{SFR}(8,24,70)$ for the A-H06 recipe. The overall trends are analogous to those observed for SFR $_{\text {TIR }}$ but with a larger scatter $(\sim 0.30$ dex $)$, i.e., the offset and rms increases with redshift up from $\Delta \mathrm{SFR}=-0.10 \pm 0.26$ dex at $z<1$ to $\Delta \mathrm{SFR}=$ $0.19 \pm 0.47$ dex at $z=2-4$.

\subsubsection{Analysis of IR-based SFRs: Best-effort Sample}

In this Section, we compare again the IR-based SFRs obtained with different methods, but this time for the best-effort sample, i.e., with estimates based on 8,24 , and $70 \mu \mathrm{m}$ data. We also present a comparison of IR-based SFRs to galaxies in common with other authors counting with better IR SED coverage (e.g., MIPS-160 or IR-spectroscopy) and therefore more reliable SFRs.

Figure 22 shows a comparison of the $\operatorname{SFRs}(8,24,70)$ obtained with each method with respect to $\operatorname{SFR}_{\mathrm{TIR}}(8,24,70$ ) (our 
reference value), as a function of SFR (left panel) and redshift (right panel). The color code is the same as in Figure 19. To simplify the comparison to the results of the previous section, the Figure also shows the ratio of $\operatorname{SFR}_{\mathrm{TIR}}(24)$ to $\operatorname{SFR}_{\mathrm{TIR}}(8,24$, $70)$, i.e., basically the values shown in the upper-left panel of Figure 20. The blue line joins the median values of the ratio of SFRs per bin of luminosity (and redshift).

The relative differences with respect to $\operatorname{SFR}_{\text {TIR }}(8,24,70)$ remain mostly unchanged with respect to what is shown in Figure 19 for estimates based on $24 \mu \mathrm{m}$ data. For SFR $\mathrm{A}-\mathrm{H} 06_{\text {the }}$ most noticeable differences are that the overall rms increases by $\sim 50 \%$ for the highest SFRs and the median ratio for $\operatorname{SFR}_{\mathrm{TIR}}(8$, $24,70)>100 M_{\odot} \mathrm{yr}^{-1}$ decreases to $\Delta \mathrm{SFR}=-0.12 \pm 0.12$ dex. For $\mathrm{SFR}_{\mathrm{B} 08}$, the median ratio $\mathrm{SFR}_{\mathrm{B} 08} / \mathrm{SFR}_{\mathrm{TIR}}$ presents a smaller tilt and a significant increment of the rms with respect to the values in the MIPS $24 \mu \mathrm{m}$ sample. Finally, SFR $_{\mathrm{R} 09}$, which only depends on the observed flux in MIPS $24 \mu \mathrm{m}$, presents the same trend at low $-z$ as in Figure 19. However, at $z \sim 2$ it tends to overestimate $\operatorname{SFR}_{\mathrm{TIR}}(8,24,70)$ because the former present similar values to $\operatorname{SFR}_{\mathrm{TIR}}(24)$ that, as shown in the previous section, overpredicts $\operatorname{SFR}_{\mathrm{TIR}}(8,24,70)$ at $z \sim 2$.

As the relative trends between the estimates of the $\operatorname{SFR}(8,24,70)$ have barely changed, our conclusions from Section 5.3.1 still apply, i.e., the values of $\mathrm{SFR}_{\mathrm{A}-\mathrm{H} 06}$ are roughly

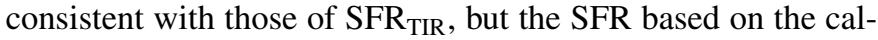
ibrations of B08 and R09 presents systematic deviations with respect to these that can be significant (up to 0.75 dex) in certain SFR and redshift intervals. In addition, we find that the typical rms of the comparison of $\operatorname{SFR}(8,24,70)$ estimates is $20 \%$ larger with respect to the previous comparison based on $24 \mu \mathrm{m}$ data. This is not surprising considering that Figure 19 shows only functional relation of each method but none of the uncertainties attached to the fit of data.

Note that the comparisons shown in Figures 19 and 22 only illustrate the expected uncertainty budget associated to the use of different SFR estimates, but they do not demonstrate that any of them provides intrinsically more accurate results. Nonetheless, having MIPS $70 \mu \mathrm{m}$ data to constrain the shape of the IR SED, it is reasonable to assume that the values of $\operatorname{SFR}_{\mathrm{TIR}}(8,24,70)$ would provide more reliable values than the other three methods. In order to verify this statement and to asses the accuracy of $\operatorname{SFR}_{\text {TIR }}(8,24,70)$, we compare the SFRs to the results from other authors based on better photometric data sets. In particular, we compare our SFRs against the values of Symeonidis et al. (2008, S08) and Huang et al. (2009). The latter studied the SFRs of a spectroscopic sample of high- $z(z \sim 1.9)$ galaxies with strong IR-emission $(f(24)>0.5 \mathrm{mJy})$. For these galaxies, the authors provide accurate SFRs estimated from a very detailed coverage of the IR SED including Spitzer/IRS spectroscopy and data at 24, 70, and $160 \mu \mathrm{m}, 1 \mathrm{~mm}$, and $1.4 \mathrm{GHz}$. In S08 the authors describe the IR properties of a $70 \mu \mathrm{m}$ selected sample restricted to galaxies detected at $160 \mu \mathrm{m}$ and having reliable spectroscopic redshifts ranging from $0.1<z<1.2(z=0.5)$.

The sources in common with S08 and Huang et al. (2009) are shown in the right panel of Figure 22 as black stars (open and closed, respectively). In addition, we show as red stars those sources which were poorly fitted to the models of CE01 and DH02 in the work of S08. We find that our values of $\operatorname{SFR}_{\mathrm{TIR}}(8,24,70)$ for the majority of the $z<1.2$ galaxies tend to underestimate the SFRs of S08 with a median (considering only black stars) difference and scatter of $\Delta \mathrm{SFR}=0.09 \pm_{0.14}^{0.20}$ dex. However, there is small group of sources for which the SFRs are systematically underestimated by $\sim 0.5 \mathrm{dex}$ or more (red stars).
In $\mathrm{S} 08$, the authors showed that, for these galaxies, the IR SED fitting to the models of CE01 and DH02 severely underfitted the data at $160 \mu \mathrm{m}$, whereas the models of Siebenmorgen \& Krügel (2007, SK07) allowed a better fit to the data (see also Symeonidis et al. 2010). As a result, the values of $L$ (TIR) obtained from the fit to models of CE01 and DH02 would be systematically lower than the estimates for SK07. These strong discrepancies in the fitting of CE01 and DH02 models do not seem to be the usual scenario (e.g., Kartaltepe et al. 2010), although some issues fitting the MIPS $160 \mu \mathrm{m}$ fluxes of local galaxies with the models of CE01 has been reported (Noll et al. 2009).

A possible explanation for this issue could be related to the fact that S08 makes use of the four IRAC bands in the SED fitting. As a result, these bands contribute significantly to the $\chi^{2}$ (more than the MIPS bands), whereas they only represent a minimum fraction of the total IR luminosity. Nonetheless, some intrinsic differences in the IR-SED of MIPS $160 \mu \mathrm{m}$ selected samples are expected, given that these are usually biased toward cold galaxies, i.e., galaxies with a relatively large (and probably not very frequent) cold dust content in comparison with the amount and emission of the warm dust featured in the models of CE01 and DH02. In summary, the differences in the SFR(8, $24,70)$ with respect to the values of S08 for these sources are most likely the result of combined SED modeling issues and selection effects.

For the rest of the sources, the $\sim 0.10$ dex offset in $\operatorname{SFR}(8$, $24,70)$ toward underestimating the values of S08 is in good agreement with the results of Kartaltepe et al. (2010, K10) for a sample of galaxies selected at $70 \mu \mathrm{m}$ (and counting with $160 \mu \mathrm{m}$ data for $\sim 20 \%$ them). The authors indicate that the estimates of $L$ (TIR) for $160 \mu \mathrm{m}$ detected sources computed without fitting that flux can be underestimated by up to 0.20 dex at $z<1$ and $\sim 0.3$ dex at higher redshifts. The authors also point out that these effect could be related to a bias in $160 \mu \mathrm{m}$ selected samples toward selecting intrinsically cooler objects (as opposed to $70 \mu \mathrm{m}$ selections). A similar statement is made in S08 based on the 160/70 flux ratios of their sample.

The comparison to the SFRs of Huang et al. (2009) for galaxies at $z \sim 2$ presents too few sources to provide a significant result. However, the overall results are in relatively good agreement within 0.3 dex. We find again that $\operatorname{SFR}_{\text {TIR }}(8$, $24,70)$ slightly underpredicts the SFRs of some galaxies, consistently with the conclusions of $\mathrm{K} 10$ for $160 \mu \mathrm{m}$ detected galaxies. Moreover, we find that the values of $\operatorname{SFR}_{\mathrm{TIR}}(24)$ for the galaxies of Huang et al. (2009) overestimates $\operatorname{SFR}(8,24$, 70 ) by a factor of $\sim 4$, following the trend shown by the blue line.

Finally, we further check the accuracy of our estimates at high- $z$, comparing them to the values of $\operatorname{SFR}_{\mathrm{TIR}}(24)$ corrected with the empirical relation of Papovich et al. (2006). This correction was conceived to mitigate the excess in the IRSFRs of high-redshift galaxies estimated from $24 \mu \mathrm{m}$ data. The correction was computed by matching the SFR(24) to the SFRs estimated from the average stacked fluxes in MIPS 24, 70, and $160 \mu \mathrm{m}$ of a sample of $z \sim 2$ galaxies. The green line in Figure 22 joins the median ratios of $\operatorname{SFR}_{\mathrm{TIR}, \mathrm{CORR}}(24) / \operatorname{SFR}_{\mathrm{TIR}}(8,24,70)$ as a function of redshift. The overall results are that the values of $\operatorname{SFR}_{\mathrm{TIR}, \mathrm{CORR}}(24)$ are a factor of $\sim 3-4$ lower than $\operatorname{SFR}_{\mathrm{TIR}}(24)$ at $z \sim 2$. As a result these estimates are also slightly lower than our predictions for $\operatorname{SFR}_{\mathrm{TIR}}(8,24,70)$. Nonetheless, the values of $\operatorname{SFR}_{\mathrm{TIR}, \mathrm{CORR}}(24)$ also agree within 0.3 dex with the SFRs of the galaxies in common with Huang et al. (2009). 
Table 7

The IRAC-3.6 + 4.5 $\mu \mathrm{m}$ Sample: Photometric Redshifts

\begin{tabular}{|c|c|c|c|c|c|c|c|c|c|c|c|}
\hline $\begin{array}{l}\text { Object } \\
\text { (1) }\end{array}$ & $\begin{array}{c}\alpha \\
(2)\end{array}$ & $\begin{array}{c}\delta \\
(3)\end{array}$ & $\begin{array}{c}\text { zphot-best } \\
\text { (4) }\end{array}$ & $\begin{array}{c}\text { zphot- } E A Z Y \\
\text { (5) }\end{array}$ & $\begin{array}{c}\text { zphot-I06 } \\
\text { (6) }\end{array}$ & $\begin{array}{c}\text { zspec } \\
(7)\end{array}$ & $\begin{array}{l}\text { qflag } \\
(8)\end{array}$ & $\begin{array}{c}\text { zphot-err } \\
\text { (9) }\end{array}$ & $\begin{array}{c}\mathrm{Qz} \\
(10)\end{array}$ & $\begin{array}{c}\mathrm{N}(\text { band }) \\
\text { (11) }\end{array}$ & $\begin{array}{c}\text { Stellarity } \\
\text { (12) }\end{array}$ \\
\hline irac003270_1 & 215.43910540 & 53.08468920 & 1.09 & 1.06 & 1.04 & 0.00000 & 2 & 0.07 & 0.31 & 16 & 0 \\
\hline irac003278 & 215.42614011 & 53.09447161 & 0.49 & 0.39 & 0.58 & 0.00000 & 0 & 0.20 & 2.58 & 16 & 7 \\
\hline irac003291_1 & 215.44058360 & 53.08123980 & 0.83 & 0.80 & 0.84 & 0.85700 & 4 & 0.02 & 0.11 & 16 & 0 \\
\hline irac003310 & 215.42129738 & 53.09430607 & 0.08 & 0.14 & 0.15 & 0.00000 & 0 & 0.07 & 0.13 & 18 & 0 \\
\hline irac003313 & 215.43553774 & 53.08200958 & 1.06 & 1.01 & 1.09 & 0.00000 & 0 & 0.08 & 0.37 & 16 & 1 \\
\hline
\end{tabular}

Notes. (1) Object unique identifier in the catalog.

$(2,3)$ Right Ascension and Declination (J2000) in degrees.

(4) Probability weighted photometric redshift. This is our default value of photometric redshift for SED fitting based estimates.

(5) Photometric redshift estimated with the code EAZY (Brammer et al. 2008) using the default template configuration and the $K$-band luminosity prior applied to the [3.6] band. The input photometric catalog is the same as for the other redshifts.

(6) Photometric redshifts as estimated in Ilbert et al. (2006a) from the (five band) $i^{\prime}$ selected catalog of the CFHTLS. This catalog overlaps with the IRAC sample in the central portion of the mosaic $\left(52.16<\delta<53.20 \& 214.04<\alpha<215^{\circ} .74\right)$.

(7) Spectroscopic redshift determination drawn from DEEP2 (Davis et al. 2007; 8000 galaxies) and (Steidel et al. 2003; LBGs at $z \gtrsim 3$ ).

(8) Quality flag of the spectroscopic redshift $(4=>99.5 \%, 3=>90 \%, 2=$ uncertain, $1=$ bad quality). Only redshifts with qflag $>2$ have been used in the analysis.

(9) Uncertainty in zphot-best(4) estimated from the $1 \sigma$ width of the probability distribution function.

(10) Reliability parameter of the photometric redshift estimated with EAZY (see Brammer et al. 2008 for more details); Good quality redshifts are in general $Q_{z} \leqslant 1$.

(11) Number of different photometric bands used in to estimate the photometric redshift with Rainbow, Column (4).

(12) Sum of all the stellarity criteria satisfied (see Section 5.4 of Paper I). A source is classified as star for Stellarity $>2$.

(This table is available in its entirety in a machine-readable form in the online journal. A portion is shown here for guidance regarding its form and content.)

\subsection{Summary of the SFRs}

The accuracy of the SFRs estimated from IR tracers up to intermediate redshifts has been demonstrated by the good agreement with the estimates based on other tracers such us dust corrected UV/optical indicator (Iglesias-Páramo et al. 2007; Salim et al. 2007, 2009). On the other hand, the systematic effects in the IR-based SFRs of the most luminous galaxies (ULIRGS) at high redshift are quite significant. Some of these issues arise from the assumptions made in the estimation of IR-based SFRs, such as the validity of the local templates at high redshift or the contribution of obscured AGNs to the IR luminosity. However, the most relevant issues arise from the lack of enough data to constrain the full IR SED, particularly for studies based on $24 \mu \mathrm{m}$ data alone. Nonetheless, the breadth and quality of the MIPS $24 \mu \mathrm{m}$ data ensure that it will continue leading multiple studies of IR-based SFR for the foreseeable future. Thus, quantifying the systematic effects between the SFRs(24) computed with different methods, and the differences in the SFRs(24) with respect to the SFRs computed from more IR data, provides a useful information.

Our analysis shows that although the values of $\operatorname{SFR}_{\mathrm{TIR}}(24)$ are consistent with those of $\mathrm{SFR}_{\mathrm{A}-\mathrm{H} 06}(24)$ within $0.3 \mathrm{dex}$ (the usual uncertainty quoted for IR-based SFRs) the values of $\mathrm{SFR}_{\mathrm{B} 08}(24)$ and $\mathrm{SFR}_{\mathrm{R} 09}(24)$ can be significantly deviated (up to $\pm 0.5 \mathrm{dex}$ ) with respect to $\operatorname{SFR}_{\mathrm{TIR}}(24)$ for certain redshift and luminosity ranges. The differences in the SFRs obtained with these methods remain mostly unchanged for $\operatorname{SFR}(8,24,70)$, and we find that the discrepancies in $\mathrm{SFR}_{\mathrm{B} 08}$ or $\mathrm{SFR}_{\mathrm{R} 09}$ with respect to $\operatorname{SFR}_{\mathrm{TIR}}(24)$ do not provide a better agreement to the SFRs of other authors computed from very detailed IR photometric data. Therefore, out of the four methods to estimate the IR-SFR discussed here, $\mathrm{SFR}_{\mathrm{TIR}}$ present (after accounting for intrinsic systematics) the more accurate results.

From the analysis of sample of MIPS $70 \mu \mathrm{m}$ detected galaxies, we find that $\operatorname{SFR}_{\mathrm{TIR}}(24)$ is reasonably consistent with the values of $\operatorname{SFR}_{\mathrm{TIR}}(8,24,70)$ up to ULIRG luminosities (typically at $z \lesssim 1.4)$ showing only a small deviation toward underestimating $\operatorname{SFR}(8,24,70)$ by 0.05 dex with an rms of 0.2 dex. However, at $z>1.5$ the agreement is significantly worse. The values of $\operatorname{SFR}_{\mathrm{TIR}}(24)$ tend to overestimate $\operatorname{SFR}(8,24,70)$ by a median value of $0.15 \pm 0.40 \mathrm{dex}$. As already pointed out by other authors, the best approach to solve this issue is to apply a correction factor that reduces the estimated values at high- $z$ (Papovich et al. 2006 or Santini et al. 2009).

The comparison of $\operatorname{SFR}_{\mathrm{TIR}}(8,24,70)$ (our best-effort SFRs) to the SFRs computed by other authors based on a better IR photometric coverage (including MIPS $160 \mu \mathrm{m}$ ) also shows an excellent agreement, proving that these estimates are robust. The overall results are consistent within 0.3 dex presenting only a small systematic deviation in $\operatorname{SFR}_{\mathrm{TIR}}(8,24,70)$ toward underestimating the values including MIPS $160 \mu \mathrm{m}$ data by -0.09 dex (mostly $z<1.2$ galaxies). Note that since this comparison is restricted to MIPS $160 \mu \mathrm{m}$ detected sources there could be some selection effects, and thus this offset might not apply for all galaxies (see, e.g., the results of K10 based on stacked fluxes in MIPS $160 \mu \mathrm{m}$ for a $70 \mu \mathrm{m}$ selected sample).

\section{DATA ACCESS}

All the data products for the 76,936 IRAC $3.6+4.5 \mu \mathrm{m}$ selected $([3.6]<23.75)$ sources in the EGS are presented here. These include (1) the photometric redshift catalog containing the estimates with Rainbow, EAZY, and from I06, when available (Table 7); (2) the stellar mass catalog containing the values estimated with each of the different modeling configurations described in Section 4 (Table 8); and (3) the SFR catalog containing the UV- and IR-based SFRs obtained with the different methods and calibrations discussed in Section 5 (Table 9). A table containing the UV-to-FIR SEDs for all these sources is presented in Paper I. The number of objects and unique identifier of this table and the tables presented in the following is the same. 
A larger version of these catalogs containing all galaxies down [3.6] $<24.75$ ( $3 \sigma$ limiting magnitude) is available through the web utility Rainbow Navigator ${ }^{7}$ (see Paper I for a more detailed description) that provides a query interface to the database containing all the data products of the multiple Rainbow tasks that we have used in the papers. Rainbow Navigator has been conceived to serve as a permanent repository for future versions of the data products in EGS, and also to similar results in other cosmological fields (such as GOODS-N and GOODS-S, presented in PG08).

\subsection{Table 7: Photometric Redshift Catalog}

These are the fields included in Table 7.

1. Object. Unique object identifier starting with irac000001. Objects labeled with an underscore plus a number (e.g., irac000356_1) are those identified as a single source in the IRAC catalog built with SExtractor, but deblended during the photometric measurement carried out with the Rainbow software (see Section 2.1). Note that, although the catalog contains 76,936 elements, the identifiers do not follow the sequence irac000001 to irac076185. This is because the catalog is extracted from a larger reference set by imposing coordinate and magnitude constraints. The table is sorted according to this unique identifier.

2. $\alpha, \delta . \mathrm{J} 2000.0$ right ascension and declination in degrees.

3. zphot-peak. Maximum likelihood photometric redshift.

4. zphot-best. Probability weighted mean photometric redshift. This is the value of $z_{\text {phot }}$ used along the paper.

5. zphot-err. $1 \sigma$ uncertainty in the photometric redshift as estimated from the $z$ PDF.

6. zphot-EAZY. Photometric redshift estimated using the $E A Z Y$ code (Brammer et al. 2008) on our SEDs with the default templates and including the $K$-band luminosity prior.

7. $Q z$. Estimate of the quality of the photometric redshifts computed with $E A Z Y$. Reliable photometric redshifts present values of $Q z \leqslant 1$ (Brammer et al. 2008).

8. zphot-I06. Photometric redshift from Ilbert et al. (2006a). These are only available for galaxies in the main region.

9. zspec. Spectroscopic redshift (set to -1 if not available).

10. qfag. Spectroscopic redshift quality flag from 1 to 4 . Sources with qfag $>3$ have a redshift reliability larger than $80 \%$.

11. $N$ (bands). Number of photometric bands used to derive the photometric redshift.

12. Stellarity. Total number of stellarity criteria satisfied. A source is classified as a star if it satisfies three or more criteria. A description of all the stellarity criteria and the accuracy of the method is given in Section 5.4 of Paper I.

\subsection{Table 8: Stellar Mass Catalog}

The stellar masses are estimated from the same templates used to compute the photometric redshifts. These templates were computed using several combination of SPS library, IMFs, and extinction laws. Our reference stellar masses are those obtained with [P01, SALP, CAL01] (see Section 3). We provide two different stellar mass estimates based on these templates depending on the redshift used during the fitting procedure, namely, zphot-best and zspec. In addition, we obtained estimates with (1) the stellar population models of Bruzual \& Charlot (2003, BC03), Maraston (2005, M05), and S. Charlot \& G.

\footnotetext{
http://rainbowx.fis.ucm.es
}

Bruzual (2011, in preparation, CB09); (2) the IMFs of Kroupa (2001, KROU) and Chabrier (2003, CHAB); and (3) the dust extinction law of Charlot \& Fall (2000, CF00). In Table 8, we give six additional stellar mass estimates obtained under different stellar population modeling assumptions, namely, [P01, KROU], [BC03, CHAB], [M05, KROU], [CB09, CHAB], [CB09, SALP], and [P01, SALP, CF00]. The extinction law in all cases except for the last is CAL01. For these stellar mass estimates we use z-fit, which is equal to zphot-best unless zspec is available.

These are the fields included in Table 8.

1. Object. Unique object identifier as in the photometric catalog.

2. $\alpha, \delta . \mathbf{J} 2000.0$ right ascension and declination in degrees.

3. Mass (best). Stellar mass $\left[\log M_{\odot}\right]$ with the associated uncertainty estimated with zphot-best using our default modeling parameters [P01, SALP, CAL01].

4. Mass (zspec). Stellar mass $\left[\log M_{\odot}\right]$ with the associated uncertainty, estimated with zspec using our default modeling parameters [P01, SALP, CAL01].

5. $z$-fit. Value of the photometric redshift used during the SED fitting with the [P01, KROU], [BC03, CHAB], [M05, KROU], [CB09, CHAB], [CB09, SALP], [P01, SALP, CF00] models. It is equal to zphot-best unless zspec is available.

6. Mass (P01, KROU). Stellar mass $\left[\log M_{\odot}\right]$ with the associated uncertainty, estimated with the modeling parameters [P01, KROU, CAL01] and zphot-fit.

7. Mass $(B C 03, C H A B)$. Stellar mass $\left[\log M_{\odot}\right]$ with the associated uncertainty, estimated with the modeling parameters [BC03, CHAB, CAL01] and zphot-fit.

8. Mass $(M 05, K R O U)$. Stellar mass $\left[\log M_{\odot}\right]$ with the associated uncertainty, estimated with the modeling parameters [M05, KROU, CAL01] and zphot-fit.

9. Mass $(C B 09, C H A B)$. Stellar mass $\left[\log M_{\odot}\right]$ with the associated uncertainty, estimated with the modeling parameters [CB09, CHAB, CAL01] and zphot-fit.

10. Mass $(C B 09, S A L P)$. Stellar mass $\left[\log M_{\odot}\right]$ with the associated uncertainty, estimated with the modeling parameters [CB09, SALP, CAL01] and zphot-fit.

11. Mass (PO1, CFO0). Stellar mass $\left[\log M_{\odot}\right]$ with the associated uncertainty, estimated with the modeling parameters [P01, SALP, CF00] and zphot-fit.

\subsection{Table 9: SFR Catalog}

The SFR catalog is presented in Table 9. The unobscured UVSFR is obtained from the best-fitting optical template modeled with [P01, SALP, CAL01]. The rest-frame IR luminosities and IR-based SFRs are computed either from the average value of the best-fitting templates from the dust emission models of Chary \& Elbaz (2001, CE01) and Dale \& Helou (2002, DH02) to MIPS $24 \mu \mathrm{m}$ data only, or the average value of the best-fitting dust emission models of CE01, DH02 and Rieke et al. (2009, R09) to IRAC-8.0, MIPS 24 and $70 \mu \mathrm{m}$ data. Only fluxes at rest-frame $\lambda>5 \mu \mathrm{m}$ are considered in this method. In both cases we use zphot-fit, which is equal to zphot-best unless zspec is available. For sources un-detected in MIPS $70 \mu \mathrm{m}$ at $z \gtrsim 0.6$ both methods provide similar results modulo the effect of the R09 templates.

Note that the SFRs has been computed for all the MIPS $24 \mu \mathrm{m}$ and $70 \mu \mathrm{m}$ detections, but only sources with $f(24)>60 \mu \mathrm{Jy}$ and $f(70)>3.5 \mathrm{mJy}$ (the $5 \sigma$ detection limit) are discussed in Section 5. In addition, sources un-detected in MIPS $24 \mu \mathrm{m}$ are 
The IRAC-3.6 + $4.5 \mu \mathrm{m}$ Sample: Stellar Mass Estimates

\begin{tabular}{|c|c|c|c|c|c|c|c|c|c|c|c|}
\hline Object & $\begin{array}{l}\alpha \\
\text { (2) }\end{array}$ & $\begin{array}{l}\delta \\
(3)\end{array}$ & $\begin{array}{c}\text { M(best) } \\
\text { M-err } \\
(4) \\
(13) \\
\end{array}$ & $\begin{array}{c}\text { M(zspec) } \\
\text { M-err } \\
(5) \\
(14) \\
\end{array}$ & $\begin{array}{l}\text { z-fit } \\
(6)\end{array}$ & $\begin{array}{c}\mathrm{M}(\mathrm{P} 01, \mathrm{KROU}) \\
\text { M-err } \\
(7) \\
(15) \\
\end{array}$ & $\begin{array}{c}\mathrm{M}(\mathrm{BC} 03, \mathrm{CHAB}) \\
\text { M-err } \\
(8) \\
(16) \\
\end{array}$ & $\begin{array}{c}\text { M(M05,KROU) } \\
\text { M-err } \\
(9) \\
(17) \\
\end{array}$ & $\begin{array}{c}\mathrm{M}(\mathrm{CB} 09, \mathrm{CHAB}) \\
\text { M-err } \\
(10) \\
(18) \\
\end{array}$ & $\begin{array}{c}\mathrm{M}(\mathrm{CB} 09, \mathrm{SALP}) \\
\text { M-err } \\
(11) \\
(19) \\
\end{array}$ & $\begin{array}{c}\mathrm{M}(\mathrm{P} 01, \mathrm{CF} 00) \\
\text { M-err } \\
(12) \\
(20) \\
\end{array}$ \\
\hline irac003270_1 & 215.43892696 & 53.08455063 & 9.79 & 9.79 & 1.09 & 9.67 & 9.64 & 9.61 & 9.61 & 9.84 & 9.98 \\
\hline & & & 0.05 & 0.05 & & 0.04 & 0.08 & 0.07 & 0.08 & 0.08 & 0.07 \\
\hline irac003278 & 215.42614011 & 53.09447161 & $\begin{array}{r}11.69 \\
0.15\end{array}$ & $\begin{array}{r}11.69 \\
0.15\end{array}$ & 0.49 & $\begin{array}{r}11.55 \\
0.14\end{array}$ & $\begin{array}{r}11.66 \\
0.17\end{array}$ & $\begin{array}{r}11.35 \\
0.16\end{array}$ & $\begin{array}{r}11.48 \\
0.18\end{array}$ & $\begin{array}{r}11.74 \\
0.15\end{array}$ & $\begin{array}{r}11.75 \\
0.17\end{array}$ \\
\hline irac003291_1 & 215.44043562 & 53.08128671 & $\begin{array}{r}10.84 \\
0.03\end{array}$ & $\begin{array}{r}10.84 \\
0.04\end{array}$ & 0.86 & $\begin{array}{r}10.97 \\
0.04\end{array}$ & $\begin{array}{r}10.64 \\
0.04\end{array}$ & $\begin{array}{r}10.30 \\
0.03\end{array}$ & $\begin{array}{r}10.57 \\
0.03\end{array}$ & $\begin{array}{r}10.63 \\
0.04\end{array}$ & $\begin{array}{r}10.48 \\
0.04\end{array}$ \\
\hline irac003310 & 215.42129738 & 53.09430607 & $\begin{array}{l}8.80 \\
0.06\end{array}$ & $\begin{array}{l}8.80 \\
0.06\end{array}$ & 0.08 & $\begin{array}{l}8.61 \\
0.06\end{array}$ & $\begin{array}{l}8.92 \\
0.08\end{array}$ & $\begin{array}{l}8.64 \\
0.08\end{array}$ & $\begin{array}{l}8.79 \\
0.05\end{array}$ & $\begin{array}{l}9.04 \\
0.05\end{array}$ & $\begin{array}{l}8.75 \\
0.06\end{array}$ \\
\hline irac003313 & 215.43553774 & 53.08200958 & $\begin{array}{l}9.94 \\
0.04\end{array}$ & $\begin{array}{l}9.94 \\
0.04\end{array}$ & 1.06 & $\begin{array}{l}9.83 \\
0.05\end{array}$ & $\begin{array}{l}9.64 \\
0.04\end{array}$ & $\begin{array}{l}9.19 \\
0.05\end{array}$ & $\begin{array}{l}9.40 \\
0.07\end{array}$ & $\begin{array}{l}9.63 \\
0.07\end{array}$ & $\begin{array}{l}9.59 \\
0.04\end{array}$ \\
\hline
\end{tabular}

Notes. (1) Object unique identifier in the catalog.

$(2,3)$ Right ascension and declination (J2000) in degrees.

(4-13) Stellar mass $\left[\log \left(M_{\odot}\right)\right]$ with the associated uncertainty, estimated with our default modeling parameters, [P01, SALP, CAL01], and zphot-best.

(5-14) Stellar mass $\left[\log \left(M_{\odot}\right)\right]$ with the associated uncertainty, estimated with our default modeling parameters, [P01, SALP, CAL01], forcing the photometric redshift to the spectroscopic value, when available.

(6) Redshift used in the fitting procedure of $(8,9,10,11$ and 12). This redshift refer to zphot-best unless the spectroscopic redshift is available; in that case the redshift is forced to the spectroscopic value.

(7-15) Stellar mass $\left[\log \left(M_{\odot}\right)\right]$ with the associated uncertainty, estimated with the modeling parameters, [P01, KROU, CAL01], and z-fit.

(8-16) Stellar mass $\left[\log \left(M_{\odot}\right)\right]$ with the associated uncertainty, estimated with the modeling parameters, [BC03, CHAB, CAL01], and z-fit.

(9-17) Stellar mass $\left[\log \left(M_{\odot}\right)\right]$ with the associated uncertainty, estimated with the modeling parameters, [M05, KROU, CAL01], and z-fit.

(10-18) Stellar mass $\left[\log \left(M_{\odot}\right)\right]$ with the associated uncertainty, estimated with the modeling parameters, [CB09, CHAB, CAL01], and z-fit.

(11-19) Stellar mass $\left[\log \left(M_{\odot}\right)\right]$ with the associated uncertainty, estimated with the modeling parameters, [CB09, SALP, CAL01], and z-fit.

(12-20) Stellar mass $\left[\log \left(M_{\odot}\right)\right]$ with the associated uncertainty, estimated with the modeling parameters, [P01, SALP, CF00], and z-fit.

(This table is available in its entirety in a machine-readable form in the online journal. A portion is shown here for guidance regarding its form and content.) 
Table $\mathbf{9}$
The IRAC-3.6 + $4.5 \mu \mathrm{m}$ Sample: IR-Luminosities and Star Formation Rate Estimates

\begin{tabular}{|c|c|c|c|c|c|c|c|c|c|c|c|c|}
\hline $\begin{array}{c}\text { Object } \\
\text { (1) }\end{array}$ & $\begin{array}{l}\alpha \\
(2)\end{array}$ & $\begin{array}{l}\delta \\
(3)\end{array}$ & $\begin{array}{c}f(24 \mu \mathrm{m}) \\
\text { err-f } \\
(4) \\
(14)\end{array}$ & $\begin{array}{c}f(70 \mu \mathrm{m}) \\
\text { err-f } \\
(5) \\
(15)\end{array}$ & $\begin{array}{l}\text { z-fit } \\
(6)\end{array}$ & $\begin{array}{c}\operatorname{SFR}(0.28) \\
(7)\end{array}$ & $\begin{array}{c}\text { SFR(R09) } \\
\text { (8) }\end{array}$ & $\begin{array}{c}L(\mathrm{TIR}, 24) \\
L(\text { TIR,best }) \\
(9) \\
(16)\end{array}$ & $\begin{array}{c}\text { SFR(TIR,24) } \\
\text { SFR(TIR,best) } \\
(10) \\
(17)\end{array}$ & $\begin{array}{c}\text { SFR(TIR,24,CE01) } \\
\text { SFR(TIR,CE01) } \\
(11) \\
(18)\end{array}$ & $\begin{array}{c}\text { SFR(B08,24) } \\
\text { SFR(B08,best) } \\
(12) \\
(19)\end{array}$ & $\begin{array}{l}\text { SFR(AH06,24) } \\
\text { SFR(AH06,best) } \\
\text { (13) } \\
(20) \\
\end{array}$ \\
\hline \multirow[t]{2}{*}{ irac003270_1 } & 215.43892696 & 53.08455063 & 55 & $\ldots$ & 1.09 & 6.0 & 21.8 & 10.93 & 14.6 & 15.1 & 17.5 & 9.4 \\
\hline & & & 13 & $\ldots$ & & & & 10.93 & 14.8 & 15.1 & 18.4 & 11.0 \\
\hline irac003278 & 215.42614011 & 53.09447161 & $\ldots$ & $\ldots$ & 0.49 & 2.0 & 1.7 & -10.34 & -3.8 & -4.7 & -8.1 & -2.2 \\
\hline \multirow[t]{2}{*}{ irac003291_1 } & 215.44043562 & 53.08128671 & $\begin{array}{c}\ldots \\
104\end{array}$ & $\begin{array}{l}\ldots \\
\ldots\end{array}$ & 0.86 & 2.5 & 17.4 & $\begin{array}{l}\ldots \\
10.92\end{array}$ & $\begin{array}{l}\ldots \\
14.4\end{array}$ & $\begin{array}{l}\ldots \\
14.6\end{array}$ & $\begin{array}{l}\ldots \\
17.5\end{array}$ & $\begin{array}{c}\ldots \\
9.3\end{array}$ \\
\hline & & & 12 & $\ldots$ & & & & 10.94 & 14.9 & 14.6 & 18.5 & 10.7 \\
\hline \multirow[t]{2}{*}{$\operatorname{irac} 003310$} & 215.42129738 & 53.09430607 & 61 & $\ldots$ & 0.08 & 0.1 & 0.0 & 8.61 & 0.1 & 0.1 & 0.3 & 0.1 \\
\hline & & & 10 & $\ldots$ & & & & 8.51 & 0.1 & 0.0 & 0.2 & 0.1 \\
\hline \multirow[t]{2}{*}{ irac003313 } & 215.43553774 & 53.08200958 & $\ldots$ & $\ldots$ & 1.06 & 3.6 & 19.9 & -10.90 & -13.8 & -14.5 & -17.3 & -8.6 \\
\hline & & & $\ldots$ & $\ldots$ & & & & $\ldots$ & $\ldots$ & $\cdots$ & $\ldots$ & $\ldots$ \\
\hline
\end{tabular}

Notes. (1) Object unique identifier in the catalog.

$(2,3)$ Right Ascension and Declination (J2000) in degrees.

$(4,14)$ Observed flux and uncertainty in MIPS $24 \mu \mathrm{m}[\mu \mathrm{Jy}]$.

$(5,15)$ Observed flux and uncertainty in MIPS $70 \mu \mathrm{m}[\mu \mathrm{Jy}]$.

(6) Redshift used in the fitting procedure. This redshift refer to zphot-best unless the spectroscopic redshift is available; in that case the redshift is forced to the spectroscopic value.

(7) UV based SFR $\left[M_{\odot} \mathrm{yr}^{-1}\right]$ estimated from the monochromatic luminosity at $2800 \AA$ A rest-frame using the calibration of Kennicutt (1998).We also refer to this value as SFR $\mathrm{UV}_{\text {.obs }}$

(8) IR based SFRs $\left[M_{\odot} \mathrm{yr}^{-1}\right]$ estimated from the observed flux in MIPS $24 \mu \mathrm{m}$ and the redshift using the formula of Rieke et al. (2009).

$(9,16)$ Total IR luminosity $\left[\log \left(L_{\odot}\right)\right]$ obtained integrating (from $8-1000 \mu \mathrm{m}$ ) the average of the best-fitting templates.

(9) is computed from the fit of MIPS $24 \mu \mathrm{m}$ data to the models of Chary \& Elbaz (2001, CE01), Dale \& Helou (2002, DH02);

(10) is computed from the fit of IRAC-8.0 and MIPS 24 and $70 \mu \mathrm{m}$ (best effort) data to the models of CE01, DH02 and Rieke et al. (2009, R09).

$(10,17)$ IR based SFRs $\left[M_{\odot} \mathrm{yr}^{-1}\right.$ ] estimated from $L(T I R)$ using the calibration of Kennicutt (1998). (10) and (17) are computed using the same combination of data and models as (9) and (16), respectively.

$(11,18)$ Same as 10 , but in this case the IR SED is fitted only with the models of CE01. (11) and (18) are computed from the fit of these models to MIPS-24 $\mu$ m data only and IRAC-8.0 and MIPS 24 and $70 \mu \mathrm{m}$ respectively.

$(12,19)$ IR based SFRs $\left.\left[M_{\odot} \mathrm{yr}^{-1}\right)\right]$ estimated from the monochromatic luminosity at $8 \mu \mathrm{m}$ rest-frame using the relation of Bavouzet et al. (2008). (12) and (19) are computed using the same combination of data and models as (9) and (16), respectively.

$(13,20)$ IR based SFRs $\left[M_{\odot} \mathrm{yr}^{-1}\right]$ estimated from the monochromatic luminosity at $24 \mu \mathrm{m}$ rest-frame using the relation of Alonso-Herrero et al. (2006). (13) and (20) are computed using the same combination of data and models as (9) and (16), respectively.

The uncertainties in the values of $L(T I R)$ and the SFRs can be as high as a factor of two. The accuracy in $(9,16)$ is limited to two decimal places and one decimal place in $(10-13,17-20)$. Negative values in the columns (9-13) indicate that the sources are non-detected in MIPS $24 \mu \mathrm{m}$. In these cases, the corresponding IR luminosity is estimated from a upper limit of $f(24)=60 \mu \mathrm{Jy}$, and the estimates in (16-20) are not computed.

(This table is available in its entirety in a machine-readable form in the online journal. A portion is shown here for guidance regarding its form and content.) 
fitted using an upper limit value of $f(24)=60 \mu \mathrm{Jy}$. In this cases the quoted $L(T I R)$ and SFRs are negative values.

1. Object. Unique object identifier as in the photometric catalog.

2. $\alpha, \delta . \mathrm{J} 2000.0$ right ascension and declination in degrees.

3. $f(24), f(70)$. Observed flux $[\mu \mathrm{Jy}]$ and uncertainties in MIPS 24 and $70 \mu \mathrm{m}$.

4. $z$-fit. Value of the redshift used during the IR SED fitting. It is equal to zphot-best unless zspec is available.

5. $L(T I R, 24)$. Total IR luminosity $\left[\log M_{\odot} \mathrm{yr}^{-1}\right]$, calculated by integrating the (average) dust emission model from $8 \mu \mathrm{m}$ to $1000 \mu \mathrm{m}$. This value is computed by fitting the observed flux in MIPS $24 \mu \mathrm{m}$ to the models of CE01 and DH02.

6. $S F R_{0.28}$. Unobscured UV-based SFR $\left[M_{\odot} \mathrm{yr}^{-1}\right]$ estimated from the rest-frame luminosity at $0.28 \mu \mathrm{m}$ interpolated in the best-fit optical template, $v L_{v}(0.28)$, using the Kennicutt (1998) calibration.

7. $S F R_{\mathrm{TIR}}(24)$. IR-based $\mathrm{SFR}\left[M_{\odot} \mathrm{yr}^{-1}\right]$, estimated from $L$ (TIR) using the calibration of Kennicutt (1998). This value is computed by fitting the observed flux in MIPS $24 \mu \mathrm{m}$ to the models of CE01 and DH02.

8. $S F R_{\mathrm{CE} 01}(24)$. Same as $\operatorname{SFR}_{\mathrm{TIR}}(24)$ but fitting the MIPS $24 \mu \mathrm{m}$ data to the models of CE01 only.

9. $S F R_{\mathrm{B} 08}(24)$. IR $\mathrm{SFR}\left[M_{\odot} \mathrm{yr}^{-1}\right]$ estimated from the restframe monochromatic luminosity at $8 \mu \mathrm{m}$ using the calibration of Bavouzet et al. (2008). This value is computed by fitting the flux in MIPS $24 \mu \mathrm{m}$ to the models of CE01 and $\mathrm{DH} 02$.

10. $\operatorname{SFR}_{\mathrm{A}-\mathrm{H} 06}(24)$. Total SFR $\left[M_{\odot} \mathrm{yr}^{-1}\right]$ estimated from the rest-frame monochromatic luminosity at $24 \mu \mathrm{m}$ using the calibration of Alonso-Herrero et al. (2006). This value is computed by fitting the observed flux in MIPS $24 \mu \mathrm{m}$ to the models of CE01 and DH02. Note that to obtain the IRSFR part of this value, the unobscured UV-SFR must be subtracted according to Equation (4).

11. $S F R_{\mathrm{R} 09}(24)$. IR SFR $\left[M_{\odot} \mathrm{yr}^{-1}\right]$ estimated from the observed flux in MIPS $24 \mu \mathrm{m}$ and the redshift using the formula of Rieke et al. (2009, Equation (14)).

12. $L(T I R), S F R_{\mathrm{TIR}}, S F R_{\mathrm{CE} 01}, S F R_{\mathrm{B} 08}, \operatorname{SFR}_{\mathrm{A}-\mathrm{H} 06}(8,24,70)$. Same as the previous values but fitting the IR SED with IRAC-8.0, MIPS 24 and $70 \mu \mathrm{m}$ data to the models of CE01, DH02, and R09. Note that $\mathrm{SFR}_{\mathrm{R} 09}$ has been omitted because its value is independent of the flux in IRAC-8.0 nor MIPS $70 \mu \mathrm{m}$.

\section{SUMMARY}

In this paper, and in Paper I (Barro et al. 2011), we have presented an IRAC-3.6 $+4.5 \mu \mathrm{m}$ selected sample in the Extended Groth Strip characterized with UV-to-FIR SEDs. The photometric catalog includes the following bands: far-UV and near-UV from GALEX, $u^{*} g^{\prime} r^{\prime} i^{\prime} z^{\prime}$ from the CFHTLS, $u^{\prime} g R i z$ from MMT and Subaru observations, BRI from CFHT12k, $V_{606}, i_{814}, J_{110}$ and $H_{160}$ from $H S T, J K$ from Palomar Observatory, CAHA and Subaru data, and [3.6]-to-[8.0], $24 \mu \mathrm{m}$, and $70 \mu \mathrm{m}$ data from Spitzer IRAC and MIPS surveys. Our catalog contains 76,936 sources down to a $85 \%$ completeness level $([3.6]<23.75)$ over an area of $0.48 \mathrm{deg}^{2}$. In addition, we have cross-correlated our sample with the redshift catalog from DEEP2, and with X-ray and VLA $20 \mathrm{~cm}$ radio data.

Paper I presented the data, the procedure to measure consistent UV-to-FIR photometry using our own dedicated software (Rainbow), and the analysis of the multi-band properties of the sample. We showed that the SEDs present the level of consistency required to characterize the intrinsic stellar populations of the galaxy. In this paper, we have presented a galaxy-bygalaxy fitting of the UV-to-FIR SEDs to stellar population and dust emission models. From the best-fitting optical and IR templates, we have estimated: (1) photometric redshifts, (2) stellar masses, and (3) SFRs. Then, we have analyzed in detail their accuracy and reliability with respect to different parameters. In the following, we present the summary of the most important results of this analysis, organized by parameter.

Photometric redshifts $\left(z_{\text {phot }}\right)$ were estimated from the comparison of the UV-to-NIR SEDs to stellar population and AGN templates. This comparison was carried out with our own dedicated software (within the Rainbow package) using $\chi^{2}$ minimization algorithm (see Pérez-González et al. 2008 for more details), and with the $E A Z Y$ code (Brammer et al. 2008). These are our main results about photometric redshifts.

1. Two new features have been included in the Rainbow photometric redshift code over the previous implementation in Pérez-González et al. (2008) to improve the quality of the estimates: (a) a zero-point re-calibration of the observed photometry and (b) the use of template error function as a weight term in the SED fitting procedure. Both features are computed simultaneously and iteratively based on the comparison of observed and synthetic photometry in a spectroscopic control sample. The results show an overall good agreement between observations and templates. The zero-point corrections are typically $\lesssim 0.1 \mathrm{mag}$ and converge after a few iterations. The overall $\mathrm{rms}$ in the residual is a factor of $\sim 2$ the median photometric uncertainty. The most noticeable discrepancies present at $\lambda>3 \mu \mathrm{m}$ and (to a lesser extent) around $250 \mathrm{~nm}$. These are the result of limitations in the stellar templates in the NIR range, and a possible excess in the strength of the dust attenuation with respect to a Calzetti et al. (2000) extinction law, respectively.

2. The comparison of our photometric redshifts to 7636 secure spectroscopic redshifts from DEEP2 and Steidel et al. (2003; LBGs at $z>3$ ) shows an overall accuracy of $\sigma_{\text {NCMAD }} \equiv 1.48 \times \operatorname{median}\left(\left|\frac{\Delta z-\text { median }(\Delta z)}{1+z_{\text {spec }}}\right|\right)=0.034$ (where $\left.\Delta z=z_{\text {phot }}-z_{\text {spec }}\right)$ and $\sigma_{\mathrm{NCMAD}}=0.046$, with $\eta=2 \%$ and $3 \%$ catastrophic outliers ( $\eta$ defined as the fraction of galaxies presenting $\sigma_{\mathrm{NCMAD}}>0.2$ ) in the EGS main region (covered by the CFHTLS) and flanking regions (covered with fewer and shallower bands), respectively. The overall scatter in $z_{\text {phot }}$ does not depend strongly on the redshift, presenting a minimum value around $z=0.5-1\left(\sigma_{\mathrm{NCMAD}}=0.028\right.$ and 0.040 in the main and flanking regions, respectively), and increasing by a factor of $\sim 1.3$ at lower and higher redshifts (up to $z<1.5$ ). At $z \sim 3$, the $z_{\text {phot }}$ accuracy for 91 LBGs with secure spectroscopy is reduced to $\sigma_{\mathrm{NCMAD}}=0.063$ and $\eta=10 \%$.

3 . The accuracy of the $z_{\text {phot }}$ is mostly independent of the [3.6] mag. However, it decreases with the optical magnitude from $\sigma_{\mathrm{NCMAD}}=0.030$ at $R=22$ to $\sigma_{\mathrm{NCMAD}}=0.060$ at $R=25$. Approximately $50 \%$ of the catastrophic outliers have $R>23.5$ and $\log \left(\chi^{2}\right)>0.6$. Approximately $60 \%$ of the sources with significantly different values of $z_{\text {best }}$ and $z_{\text {peak }}\left(\left|z_{\text {best }}-z_{\text {peak }}\right| /(1+z)>0.2\right)$ are catastrophic outliers.

4. The $z_{\text {phot }}$ statistics for the 1995 and 262 spectroscopic galaxies detected in MIPS $24 \mu \mathrm{m}(f(24)>60 \mu \mathrm{Jy})$ and $70 \mu \mathrm{m}(f(70)>3.5 \mathrm{mJy})$ in the main region are similar to the rest of the sample with $\sigma_{\mathrm{NCMAD}}=0.033, \eta=3 \%$, and 
$\sigma_{\mathrm{NCMAD}}=0.045, \eta=1 \%$, respectively. The accuracy for the $142 \mathrm{X}$-ray sources is similar $\left(\sigma_{\mathrm{NCMAD}}=0.038\right)$ although with larger fraction of outliers $(\eta=10 \%)$, probably as a result of some degree of contamination by the AGN, for which reliable $z_{\text {phot }}$ are difficult to estimate based on stellar templates. The worst results are found for a very few (12) power-law galaxies (PLGs, identified as obscured AGNs): $\sigma_{\text {NCMAD }}=0.052(\eta=17 \%)$. We also note that sources with increasing fluxes in the IRAC bands $\left(f_{[3.6]}<f_{[4.5]}<\right.$ $\left.f_{[5.8]}<f_{[8.0]}\right)$ make up for up to $15 \%$ of the total number of outliers.

5. The $z_{\text {phot }}$ Rainbow are in good agreement with those from the $i^{\prime}$-band-selected catalog of Ilbert et al. (2006a, I06), which overlaps with our sample in the main region. For the 5454 galaxies in common between the two catalogs with [3.6] $<23.75$ and $i^{\prime}<24.5$, the accuracy of the $z_{\text {phot }}$ at $z<1$ is roughly the same, $\sigma_{\mathrm{NCMAD}}=0.035$. At higher redshifts, our larger band coverage (mostly in the NIR) provides more accurate results and less severe systematic errors and uncertainties. In particular, for galaxies at $z \sim$ 3 (the LBG sub-sample), the outlier fraction in I06 is $46 \%$ for only $9 \%$ in Rainbow. Our $z_{\text {phot }}$ catalog and the one presented in I06 are complementary: whereas the NIRselected sample detected more galaxies at high- $z$, which are too faint in the optical to be included in the I06 catalog, the IRAC catalog misses a population of low-mass galaxies at $z<1$ which are recovered by the $i^{\prime}$-band selection in I06.

6. We showed that the photometric catalog provides robust SEDs by obtaining a different realization of the $z_{\text {phot }}$ catalog with similar quality using the code $E A Z Y$ (Brammer et al. 2008). In particular, these alternative photometric redshifts are slightly more accurate for the sources in the main region, particularly at $z<0.5$, whereas they present a larger scatter in the flanking regions. Moreover, these $z_{\text {phot }}$ exhibit a slightly larger systematic deviation $(\Delta z /(1+z)=0.019$ and 0.027 in the main and flanking regions, respectively) than the $z_{\text {phot }}$ computed with Rainbow.

7. We further tested the accuracy of our $z_{\text {phot }}$ by checking the number densities and $z_{\text {phot }}$ distributions of a sub-sample of (NIR-selected) s-BzK $\left(\rho=5.0 \operatorname{arcmin}^{2} ; \tilde{z}=1.89\right), \mathrm{p}-\mathrm{BzK}$ $\left(\rho=0.5 \operatorname{arcmin}^{2} ; \tilde{z}=1.85\right)$, and DRG $\left(\rho=1.4 \operatorname{arcmin}^{2}\right.$; $\tilde{z}=2.47)$. These are in relatively good agreement with the results from the literature down to $K_{\mathrm{VEGA}}<21$. The most significant difference is an excess of $\sim 1.5$ in the density sBzKs, which could be caused by an overdensity of galaxies at $z \sim 1.5$.

8. The median redshift of the $([3.6]<23.75)$ sample, $\tilde{z}=1.2$, is consistent with that of the flux limited samples of PérezGonzález et al. (2008) and Ilbert et al. (2009) in different fields.

Stellar masses for the whole sample were obtained in a galaxy-by-galaxy basis by fitting the optical-to-NIR SEDs to SPS models. In addition, we analyzed the effects of the choice of different SPS libraries, IMFs, and dust extinction laws on our estimations. For that we considered a reference set of assumptions to which several combinations of input parameters were compared. This reference stellar masses were obtained with the PEGASE 2.0 (Fioc \& Rocca-Volmerange 1997) SPS models (P01), a Salpeter (1955) IMF (SALP), and the Calzetti et al. (2000, CALZ01) extinction law. We compared these estimations with those obtained with: (1) the stellar population models of Bruzual \& Charlot (2003, BC03), Maraston (2005, M05), and S. Charlot \& G. Bruzual (2011, in preparation,
CB09); (2) the IMFs of Kroupa (2001, KROU) and Chabrier (2003, CHAB); (3) the dust extinction law of Charlot \& Fall (2000, CF00). These are our main results about stellar masses.

1. From the comparison of the stellar masses estimated with photometric and spectroscopic redshifts we find a $1 \sigma$ uncertainty of $\sim 0.2$ dex. The distribution of stellar masses as a function of redshift for our default modeling assumptions shows that $90 \%$ of the galaxies present $\log (M)>$ $10 M_{\odot}$ at $z>2$, at the limiting magnitude of our sample $([3.6]<23.75)$.

2. We quantified the impact of the choice of different IMFs in the estimated stellar masses. For the CB09 models, we found that the use of a SALP, KROU, or CHAB IMF introduces constant offsets (with a very small scatter) in the estimated stellar masses: $\Delta \log (M)[\mathrm{SALP}-\mathrm{KROU}]=$ 0.19 dex and $\Delta \log (\mathrm{M})[\mathrm{CHAB}-\mathrm{KROU}]=-0.04$ dex. For the models of P01, the difference for a SALP and KROU IMFs depends on the mass, ranging from $\Delta \log (M)=$ 0.03 dex for masses lower than $\log (M)=10 \mathcal{M}_{\odot}$ to $0.13 \mathrm{dex}$ above that threshold.

3. We quantified the impact of using different SPS codes in the estimated stellar masses. We found that the new CB09 models predict slightly lower masses than the older version, BC03, by $\Delta \log (M)=0.04 \pm_{0.15}^{0.28}$ dex. Our stellar masses estimated with the P01 models are on average larger than those obtained with the CB09 models (for a KROU IMF) by $\Delta \log (M)=0.15 \pm 0.26 \mathrm{dex}$. The estimates with the P01 library are also larger than those with the M05 SPS by $\Delta \log (M)=0.39 \pm 0.34 \mathrm{dex}$. We found slightly lower values of this offset for galaxies with $\log (M)>10 \mathcal{M}_{\odot}$ ( $\sim 0.30 \mathrm{dex})$. Our default modeling assumptions, [P01, SALP, CAL01], predict comparatively the largest stellar masses. Accounting for all systematic offsets, all models are roughly consistent within a factor of 2-3.

4. We quantified the effect of using different treatments of the dust extinction by comparing the stellar masses estimated with a CAL01 and CF00 extinction laws. The median result is a small systematic deviation of 0.03 dex toward smaller values when using CAL01, and an rms of $\sim 0.20$ dex. This suggest that the different treatments of the dust attenuation do not play a major role in the estimate of the stellar masses.

5. The comparison of our results with several stellar mass catalogs already published in EGS revealed a good agreement despite the differences in the modeling technique and in the photometric data set. We found a median offset and scatter of $\Delta \log (M)=-0.07 \pm 0.21 \mathrm{dex}$ and $\Delta \log (M)=0.10 \pm$ 0.25 dex with respect to the catalogs of stellar masses published by Bundy et al. (2006) and Trujillo et al. (2007), respectively.

SFRs were estimated for all galaxies in our sample following a variety of procedures. First, we calculated the unobscured SFR (the star formation which is directly observable in the UV/ optical) from the observed luminosity at $280 \mathrm{~nm}\left(\mathrm{SFR}_{\mathrm{UV} \text {,obs }}\right)$. To get the total SFR of a galaxy, the former value must be added to the SFR which is not directly measurable in the UV/optical because of the extinction by dust. We calculated this SFR from the IR data taken by Spitzer/IRAC and MIPS at $24 \mu \mathrm{m}$ and $70 \mu \mathrm{m}$ (if available). The general procedure consists on fitting the IR photometry at rest-frame wavelengths $\lambda>5 \mu \mathrm{m}$ (usually involving 8,24, and $70 \mu \mathrm{m}$ data) to the dust emission templates of Chary \& Elbaz (2001, CE01) and Dale \& Helou (2002, DH02) and Rieke et al. (2009), but we also performed some test by fitting only MIPS $24 \mu \mathrm{m}$ data to the models of CE01 and DH02. 
From the best fit to the models, the IR-based SFR for each object was estimated with four different methods: (1) the total infrared luminosity, $L$ (TIR), integrated from 8 to $1000 \mu \mathrm{m}$ transformed to an SFR with the factor published by Kennicutt (1998). (2) The rest-frame monochromatic luminosity at $8 \mu \mathrm{m}\left(\mathrm{SFR}_{\mathrm{B} 08}\right)$ transformed to $L$ (TIR) and SFR using the empirical relation described in Bavouzet et al. (2008) and the Kennicutt factor. (3) The empirical relation given in Alonso-Herrero et al. (2006) between the rest-frame monochromatic luminosity at $24 \mu \mathrm{m}$ and the SFR ( SFR $\left._{\mathrm{A}-\mathrm{H} 06}\right)$. (4) Using Equation (14) of Rieke et al. (2009) that relates the SFR ( SFR $\left._{\mathrm{R} 09}\right)$ to the observed flux in the MIPS $24 \mu \mathrm{m}$ band and the redshift. The monochromatic and integrated luminosities were computed from the average value of the best-fit templates. These are our main results about SFRs.

1. We quantified the differences in the IR-based SFRs obtained with the four methods based on the fit to MIPS $24 \mu \mathrm{m}$ data only. The SFR estimates with the models of CE01 and DH02 are compatible within a factor of two, presenting a maximum difference around $z \sim 1.5$. The estimates of $\mathrm{SFR}_{\mathrm{TIR}}$ and $\mathrm{SFR}_{\mathrm{A}-\mathrm{H} 06}$ are roughly consistent $(\triangle \mathrm{SFR} \sim$ $-0.18 \pm 0.05$ dex) when the contribution of $S_{F} R_{U V}$,obs is small. $\mathrm{SFR}_{\mathrm{B} 08}$ gives systematically lower values than $\mathrm{SFR}_{\mathrm{TIR}}$ for $\mathrm{SFR}>20 M_{\odot} \mathrm{yr}^{-1}$ and $z>1$, and higher values for lower redshifts and SFRs. The difference exceeds a factor of five for SFR $>1000 M_{\odot} \mathrm{yr}^{-1}$. The overall agreement between $\mathrm{SFR}_{\mathrm{TIR}}$ and $\mathrm{SFR}_{\mathrm{R} 09}$ is rather poor, except at $z>1.8$ where the differences are lower than 0.05 dex. The reasons for these discrepancies can be found in the differences in the relative emission of the cold and warm dust, and in the strength of the PAH and silicate absorption. These characteristics can vary by up to a factor of $\sim 5$ from one set of templates to the other.

2. For each of the methods to estimate the SFR, we studied the effect having a better constrained IR SED comparing the SFRs computed from IRAC+MIPS, $\operatorname{SFR}(8,24,70)$, and just MIPS $24 \mu \mathrm{m}$, SFR(24). At low- $z$, the median values of $\operatorname{SFR}_{\mathrm{TIR}}(24)$ and $\mathrm{SFR}_{\mathrm{A}-\mathrm{H} 06}(24)$ tend to underestimate $\operatorname{SFR}(8,24,70)$ by 0.05 and $0.10 \mathrm{dex}$, respectively, with an rms of $\sim 0.2-0.3$ dex. At $z \sim 2$, the estimates from these two methods based on $24 \mu \mathrm{m}$ data are only on average $\sim 0.20$ dex larger than those obtained with $\operatorname{SFR}(8,24,70)$. $\mathrm{SFR}_{\mathrm{B} 08}(24)$ presents the opposite trend, giving larger values than $\operatorname{SFR}_{\mathrm{B} 08}(8,24,70)$ at $z \leqslant 1$ (up to $0.18 \mathrm{dex}$ ), but remaining mostly unchanged at higher redshifts.

3. The relative differences between each of the methods to estimate the IR-based SFRs with respect $\mathrm{SFR}_{\mathrm{TIR}}$ (described in the first item) remain mostly unchanged when using $\operatorname{SFRs}(8,24,70)$. The values of $\operatorname{SFR}_{\mathrm{TIR}}(8,24,70)$ (besteffort estimate) for a sample of galaxies in common with Symeonidis et al. (2008) and Huang et al. (2009), who counted with more photometric fluxes in the FIR and (sub-)mm range, presented a relatively good agreement within $\sim 0.3$ dex. At $z<1.2$ we find a small deviation of 0.09 dex in $\operatorname{SFR}_{\mathrm{TIR}}(8,24,70)$ toward underestimating the SFR of MIPS-160 detected galaxies. Larger discrepancies, up to $0.5 \mathrm{dex}$, might arise for individual galaxies due to the use of different template sets.

In the context of studies of galaxy evolution, our catalog provides a self-consistent sample with a very detailed characterization of the systematic uncertainties suitable for multiple scientific purposes. It is also an alternative to other catalogs providing only photometry, redshifts, or stellar parameters alone.
Furthermore, our photometric catalog itself provides a reference point for independent analysis of the stellar populations.

The multi-band photometric catalog presented in Paper I, jointly with the photometric redshifts and estimated stellar parameters presented here are publicly available. We have developed a Web interface, named Rainbow Navigator ${ }^{8}$, that provides full access to the imaging data and estimated parameters and allows several other data handling functionalities.

We thank the referee for providing constructive comments and help in improving the contents of this paper. We thank G. Bruzual and S. Charlot for allowing us to use their models prior to publication. We acknowledge support from the Spanish Programa Nacional de Astronomía y Astrofísica under grants AYA 2006-02358 and AYA 2006-15698-C02-02. P.G.P.-G. acknowledges support from the Ramón y Cajal Program financed by the Spanish Government and the European Union. Partially funded by the Spanish MICINN under the Consolider-Ingenio 2010 Program grant CSD2006-00070: First Science with the GTC. Support was also provided by NASA through contract no. 1255094 issued by JPL/Caltech. This work is based in part on observations made with the Spitzer Space Telescope, which is operated by the Jet Propulsion Laboratory, Caltech under NASA contract 1407. Observations reported here were obtained at the MMT Observatory, a joint facility of the Smithsonian Institution and the University of Arizona. GALEX is a NASA Small Explorer launched in 2003 April. We gratefully acknowledge NASA's support for construction, operation, and scientific analysis of the GALEX mission. This research has made use of the NASA/IPAC Extragalactic Database (NED) which is operated by the Jet Propulsion Laboratory, California Institute of Technology, under contract with the National Aeronautics and Space Administration. Based in part on data collected at Subaru Telescope and obtained from the SMOKA, which is operated by the Astronomy Data Center, National Astronomical Observatory of Japan.

\section{REFERENCES}

Alonso-Herrero, A., et al. 2004, ApJS, 154, 155

Alonso-Herrero, A., et al. 2006, ApJ, 650, 835

Assef, R. J., et al. 2010, ApJ, 713, 970

Barro, G., et al. 2011, ApJS, 193, 13

Bavouzet, N., et al. 2008, A\&A, 479, 83

Bell, E. F., McIntosh, D. H., Katz, N., \& Weinberg, M. D. 2003, ApJ, 585, L117

Bell, E. F., et al. 2005, ApJ, 625, 23

Benítez, N. 2000, ApJ, 536, 571

Bertin, E., \& Arnouts, S. 1996, A\&AS, 117, 393

Blanc, G. A., et al. 2008, ApJ, 681, 1099

Bolzonella, M., Miralles, J., \& Pelló, R. 2000, A\&A, 363, 476

Bouwens, R. J., et al. 2009, ApJ, 705, 936

Brammer, G. B., van Dokkum, P. G., \& Coppi, P. 2008, ApJ, 686, 1503

Brodwin, M., et al. 2006, ApJ, 651, 791

Bruzual, G. 2007, in ASP Conf. Ser. 374, From Stars to Galaxies: Building the Pieces to Build Up the Universe, ed. A. Vallenari et al. (San Francisco, CA: ASP), 303

Bruzual, G., \& Charlot, S. 2003, MNRAS, 344, 1000

Bundy, K., et al. 2006, ApJ, 651, 120

Burgarella, D., et al. 2007, MNRAS, 380, 986

Barro, G., et al. 2009, A\&A, 494, 63

Calzetti, D., et al. 2000, ApJ, 533, 682

Calzetti, D., et al. 2007, ApJ, 666, 870

Caputi, K. I., et al. 2006, ApJ, 637, 727

Cardamone, C. N., et al. 2010, ApJS, 189, 270

Cardelli, J. A., Clayton, G. C., \& Mathis, J. S. 1989, ApJ, 345, 245

\footnotetext{
8 http://rainbowx.fis.ucm.es
} 
Chabrier, G. 2003, PASP, 115, 763

Charlot, S., \& Fall, S. M. 2000, ApJ, 539, 718

Chary, R., \& Elbaz, D. 2001, ApJ, 556, 562

Coleman, G. D., Wu, C., \& Weedman, D. W. 1980, ApJS, 43, 393

Colina, L., \& Bohlin, R. C. 1994, AJ, 108, 1931

Collister, A. A., \& Lahav, O. 2004, PASP, 116, 345

Conroy, C., \& Gunn, J. E. 2010, ApJ, 712, 833

Conroy, C., Gunn, J. E., \& White, M. 2009, ApJ, 699, 486

Conroy, C., White, M., \& Gunn, J. E. 2010, ApJ, 708, 58

Conselice, C. J., et al. 2007, ApJ, 660, L55

Conselice, C. J., et al. 2008, MNRAS, 383, 1366

Coupon, J., et al. 2009, A\&A, 500, 981

Daddi, E., et al. 2004, ApJ, 617, 746

Daddi, E., et al. 2007, ApJ, 670, 156

Dale, D. A., \& Helou, G. 2002, ApJ, 576, 159

Dale, D. A., et al. 2005, ApJ, 633, 857

Davis, M., et al. 2007, ApJ, 660, L1

De Lucia, G., \& Blaizot, J. 2007, MNRAS, 375, 2

Donley, J. L., Rieke, G. H., Pérez-González, P. G., Rigby, J. R., \& Alonso-

Herrero, A. 2007, ApJ, 660, 167

Elbaz, D., et al. 2010, A\&A, 518, L29

Fioc, M., \& Rocca-Volmerange, B. 1997, A\&A, 326, 950

Fontana, A., et al. 2006, A\&A, 459, 745

Förster Schreiber, N. M., et al. 2006, AJ, 131, 1891

Franx, M., et al. 2003, ApJ, 587, L79

Gawiser, E., et al. 2006, ApJS, 162, 1

Grazian, A., et al. 2006a, A\&A, 449, 951

Grazian, A., et al. 2006b, A\&A, 453, 507

Grazian, A., et al. 2007, A\&A, 465, 393

Hartley, W. G., et al. 2008, MNRAS, 391, 1301

Hartley, W. G., et al. 2010, MNRAS, 407, 1212

Hildebrandt, H., Wolf, C., \& Benítez, N. 2008, A\&A, 480, 703

Hopkins, A. M., \& Beacom, J. F. 2006, ApJ, 651, 142

Huang, J., et al. 2004, ApJS, 154, 44

Huang, J., et al. 2005, ApJ, 634, 137

Huang, J., et al. 2009, ApJ, 700, 183

Iglesias-Páramo, J., et al. 2007, ApJ, 670, 279

Ilbert, O., et al. 2006a, A\&A, 457, 841

Ilbert, O., et al. 2006b, A\&A, 453, 809

Ilbert, O., et al. 2009, ApJ, 690, 1236

Ilbert, O., et al. 2010, ApJ, 709, 644

Ivison, R. J., et al. 2007, ApJ, 660, L77

Kajisawa, M. 2006, PASJ, 58, 951

Kannappan, S. J., \& Gawiser, E. 2007, ApJ, 657, L5

Kartaltepe, J. S., et al. 2010, ApJ, 709, 572

Kennicutt, R. C., Jr. 1998, ARA\&A, 36, 189

Kennicutt, R. C., et al. 2009, ApJ, 703, 1672

Kinney, A. L., et al. 1996, ApJ, 467, 38

Kriek, M., et al. 2008, ApJ, 677, 219

Kron, R. G. 1980, ApJS, 43, 305

Kroupa, P. 2001, MNRAS, 322, 231

Kurucz, R. L. 1992, in IAU Symp. 149, The Stellar Populations of Galaxies, ed. B. Barbuy \& A. Renzini (Cambridge: Cambridge Univ. Press), 225

Labbé, I., et al. 2003, AJ, 125, 1107

Laird, E. S., et al. 2009, ApJS, 180, 102

Magdis, G. E., et al. 2008, MNRAS, 386, 11

Magnelli, B., et al. 2008, ApJ, 681, 258

Maraston, C. 1998, MNRAS, 300, 872
Maraston, C. 2005, MNRAS, 362, 799

Maraston, C., et al. 2006, ApJ, 652, 85

Maraston, C., et al. 2010, MNRAS, 407, 830

Marchesini, D., et al. 2009, ApJ, 701, 1765

Marcillac, D., et al. 2006, A\&A, 451, 57

McCracken, H. J., et al. 2010, ApJ, 708, 202

Mentuch, E., et al. 2009, ApJ, 706, 1020

Morrissey, P., et al. 2007, ApJS, 173, 682

Muzzin, A., et al. 2009, ApJ, 701, 1839

Noll, S., Pierini, D., Pannella, M., \& Savaglio, S. 2007, A\&A, 472, 455

Noll, S., et al. 2009, A\&A, 507, 1793

Noterdaeme, P., Ledoux, C., Srianand, R., Petitjean, P., \& Lopez, S. 2009, A\&A, 503,765

Oyaizu, H., Lima, M., Cunha, C. E., Lin, H., \& Frieman, J. 2008, ApJ, 689, 709

Panter, B., Jimenez, R., Heavens, A. F., \& Charlot, S. 2007, MNRAS, 378, 1550

Papovich, C., \& Bell, E. F. 2002, ApJ, 579, L1

Papovich, C., et al. 2006, ApJ, 640, 92

Papovich, C., et al. 2007, ApJ, 668, 45

Pérez-González, P. G., et al. 2005, ApJ, 630, 82

Pérez-González, P. G., et al. 2006, ApJ, 648, 987

Pérez-González, P. G., et al. 2008, ApJ, 675, 234

Pérez-González, P. G., et al. 2010, A\&A, 518, L15

Polletta, M., et al. 2007, ApJ, 663, 81

Quadri, R., et al. 2007, AJ, 134, 1103

Reddy, N. A., Steidel, C. C., Erb, D. K., Shapley, A. E., \& Pettini, M. 2006, ApJ, 653,1004

Reddy, N. A., et al. 2005, ApJ, 633, 748

Reddy, N. A., et al. 2008, ApJS, 175, 48

Rieke, G. H., et al. 2009, ApJ, 692, 556

Rigby, J. R., et al. 2008, ApJ, 675, 262

Rigopoulou, D., et al. 2006, ApJ, 648, 81

Salim, S., et al. 2007, ApJS, 173, 267

Salim, S., et al. 2009, ApJ, 700, 161

Salimbeni, S., Fontana, A., Giallongo, E., Grazian, A., Menci, N., Pentericci, L., \& Santini, P. 2009, in AIP Conf. Ser. 1111, ed. G. Giobbi et al. (Melville, NY: AIP), 207

Salpeter, E. E. 1955, ApJ, 121, 161

Santini, P., et al. 2009, A\&A, 504, 751

Schlegel, D. J., Finkbeiner, D. P., \& Davis, M. 1998, ApJ, 500, 525

Siebenmorgen, R., \& Krügel, E. 2007, A\&A, 461, 445

Silva, L., Granato, G. L., Bressan, A., \& Danese, L. 1998, ApJ, 509, 103

Smith, J. D. T., et al. 2007, ApJ, 656, 770

Springel, V., et al. 2005, Nature, 435, 629

Steidel, C. C., et al. 2003, ApJ, 592, 728

Steidel, C. C., et al. 2004, ApJ, 604, 534

Symeonidis, M., et al. 2008, MNRAS, 385, 1015

Symeonidis, M., et al. 2010, MNRAS, 403, 1474

Taylor, E. N., et al. 2009, ApJ, 694, 1171

Trujillo, I., et al. 2007, MNRAS, 382, 109

van der Wel, A., et al. 2006, ApJ, 652, 97

van Dokkum, P. G., et al. 2008, ApJ, 677, L5

van Dokkum, P. G., et al. 2009, PASP, 121, 2

Walcher, C. J., et al. 2008, A\&A, 491, 713

Werner, M. W., et al. 2004, ApJS, 154, 309

Wild, V., et al. 2007, MNRAS, 381, 543

Wolf, C., et al. 2003, A\&A, 401, 73

Wuyts, S., et al. 2008, ApJ, 682, 985 